\title{
Software Toolkit for HFE-based Multivariate Schemes
}

\author{
Jean-Charles Faugère ${ }^{1,3,2}$, Ludovic Perret ${ }^{1,2,3}$ and Jocelyn Ryckeghem ${ }^{2,3}$ \\ 1 CryptoNext \\ 2 Sorbonne Université, CNRS, Laboratoire d'Informatique de Paris 6, LIP6, \\ Équipe PolSys, 4 place Jussieu, F-75005, Paris, France \\ ${ }^{3}$ INRIA Paris \\ jcf@cryptonext-security.com, ludovic.perret@cryptonext-security.com, \\ jocelyn.ryckeghem@lip6.fr
}

\begin{abstract}
In 2017, NIST shook the cryptographic world by starting a process for standardizing post-quantum cryptography. Sixty-four submissions have been considered for the first round of the on-going NIST Post-Quantum Cryptography (PQC) process. Multivariate cryptography is a classical post-quantum candidate that turns to be the most represented in the signature category. At this stage of the process, it is of primary importance to investigate efficient implementations of the candidates. This article presents MQsoft, an efficient library which permits to implement HFE-based multivariate schemes submitted to the NIST PQC process such as GeMSS, Gui and DualModeMS. The library is implemented in C targeting Intel 64-bit processors and using avx2 set instructions. We present performance results for our library and its application to GeMSS, Gui and DualModeMS. In particular, we optimize several crucial parts for these schemes. These include root finding for HFE polynomials and evaluation of multivariate quadratic systems in $\mathbb{F}_{2}$. We propose a new method which accelerates root finding for specific HFE polynomials by a factor of two. For GeMSS and Gui, we obtain a speed-up of a factor between 2 and 19 for the keypair generation, between 1.2 and 2.5 for the signature generation, and between 1.6 and 2 for the verifying process. We have also improved the arithmetic in $\mathbb{F}_{2^{n}}$ by a factor of 4 compared to the NTL library. Moreover, a large part of our implementation is protected against timing attacks.
\end{abstract}

Keywords: MQsoft · efficient software implementation $\cdot$ constant-time $\cdot \mathrm{HFEv}-\cdot \mathrm{GeMSS}$ · Gui · DualModeMS · root finding · binary fields

\section{Introduction}

The recent progress on the development of quantum computers has motivated NIST [oST17] to start a standardization process for post-quantum cryptography. In this paper, we are interested in the category of multivariate signature schemes. The choice of the best candidate is based on its security and its performance. At this stage, the security parameters of the candidates are already fixed, but the implementations can be improved. In this article, we study the efficient implementation of multivariate schemes.

We present here software tools that allow the efficient implementation of HFE-based schemes (using arithmetic in $\mathbb{F}_{2^{n}}$ ). In particular, our software tools allow to speed-up the GeMSS $\left[\mathrm{CFMR}^{+} 17\right]$, Gui $\left[\mathrm{CDP}^{+} 17\right]$ and DualModeMS [FPR17] signature schemes, which are candidates submitted to the NIST post-quantum cryptography standardization process [oST17]. The advantage of $\mathbb{F}_{2^{n}}$ is that each element can be represented as a vector of 
bits, which corresponds to the architecture of binary computers and can be naturally improved by vector instructions. The signature generation requires arithmetic in $\mathbb{F}_{2^{n}}[X]$, and its implementation is already provided by various libraries. Among the best, NTL [Sho03] provides high quality implementations of state-of-the-art algorithms. But these algorithms are not specialized for the case of sparse polynomials in $\mathbb{F}_{2^{n}}[X]$. Moreover, the implementations are not constant-time and so are vulnerable to timing attacks. For these reasons, we need to adapt the algorithms used. We have chosen to create a new library, which is based on constant-time arithmetic in $\mathbb{F}_{2^{n}}$. Unlike NTL, which offers a general implementation, our library is specialized for a value of $n$, permitting more efficient arithmetic. Moreover, we exploit the sparse polynomial structure to improve the performance. More generally, our implementation uses the Intel vector instructions to obtain interesting speed-ups.

Our library also supports DualModeMS [FPR17], which is a candidate in the NIST PQC standardization process. It is a modified HFE-based signature scheme which permits to decrease the size of the public-key, but by increasing the size of the signature. The parameters are chosen to minimize the sum of both sizes. By improving the implementation of HFE-based schemes, we automatically improve the implementation of DualModeMS.

Evaluation of multivariate quadratic systems. Many multivariate cryptosystems require to evaluate a multivariate quadratic system (MQS) to encrypt data or verify a signature (e.g. $\left.\left[\mathrm{CDP}^{+} 17, \mathrm{CFMR}^{+} 17, \mathrm{CHR}^{+} 16\right]\right)$. Encryption uses secret data and should be performed in constant-time, whereas verification is a public process and does not have this constraint. In HFEv- signature schemes, the evaluation step is the main part of verification. Efficient implementations of evaluation have been studied in [BBG06, $\mathrm{CCC}^{+} 09, \mathrm{CHR}^{+} 16, \mathrm{CLP}^{+}$18]. The authors of $[\mathrm{BBG} 06]$ propose different strategies for the evaluations in $\mathbb{F}_{2}, \mathbb{F}_{2^{4}}$ and $\mathbb{F}_{2^{8}}$. In $\left[\mathrm{CCC}^{+} 09\right]$, the evaluation is vectorized with ssse 3 instructions in $\mathbb{F}_{31}, \mathbb{F}_{16}$ and $\mathbb{F}_{256}$. In $\left[\mathrm{CHR}^{+} 16\right]$, the authors propose to optimize the evaluation in $\mathbb{F}_{31}$ and $\mathbb{F}_{2^{256}}$ by evaluating the public-key equations one-by-one. Their implementation is vectorized with the avx2 instructions set. In $\left[\mathrm{CLP}^{+} 18\right]$, the authors present a faster evaluation with the same instructions set. To do so, they use a "monomial representation" of the public-key: for each monomial, the corresponding coefficients in each equation are stored together. We optimize the evaluation with this representation to obtain new speed records.

Root finding of a HFE polynomial. The main part of the signature generation in Gui and GeMSS is to find the roots of a polynomial $F$ in $\mathbb{F}_{2^{n}}[X]$ with a specific form. Root finding is a fundamental problem in computer algebra with various applications in discrete mathematics. A survey of the main root finding methods can be found in [vzGG13]. Recently, the successive resultants algorithm (SRA) [Pet14] has been proposed to find the roots of a polynomial in small characteristic, and this work has been extended for split polynomials in general finite fields. In [DPP16], root finding is improved for split and separable polynomials, when the cardinality of multiplicative group is smooth.

In the case of the HFE polynomial $F$ in $\mathbb{F}_{2^{n}}[X], F$ has a sparse structure and its coefficients are in a field of small characteristic. Moreover, the number of roots is generally small (it is almost always less than 10 for our parameters). The main challenge is to exploit the sparse structure of $F$ to improve the complexity of the root finding: it should depend on the number of coefficients of $F$ and not on its degree. In practice, the Berlekamp algorithm [vzGG13, Algorithm 14.15] is used, which computes $\operatorname{GCD}\left(F,\left(X^{2^{n}}-X\right) \bmod F\right)$. The most costly task is the computation of $X^{2^{n}} \bmod F$, also called the Frobenius map, and the HFE structure can be exploited during the modular reduction by $F$. In $\left[\mathrm{PCY}^{+} 15\right]$, the authors propose a method to compute the Frobenius map with multi-squaring tables, which is interesting when the degree of $F$ is (approximately) smaller than $n$. We study how to implement the Frobenius map efficiently, optimizing as a function of the parameters. 
Arithmetic in $\mathbb{F}_{2^{n}}$. Arithmetic in $\mathbb{F}_{2^{n}}$ is a critical part of the root finding algorithm, because all operations in $\mathbb{F}_{2^{n}}[X]$ require it, and is studied in [ALH10b, ALH10a, TFA ${ }^{+} 11$, BG13]. In particular, multiplication in $\mathbb{F}_{2^{n}}$ is the most critical operation. This is a well-known task and is studied in [BGTZ08, DG17, MS17, CCK $\left.{ }^{+} 17\right]$. We choose here to use the PCLMULQDQ instruction (Section 1.7) to obtain an efficient implementation. This instruction computes the product of two binary polynomials, each of degree strictly less than 64 .

\section{Organization of the Paper and Main Results}

We present MQsoft [MQs18]: an efficient open-source library in C for HFE-based schemes such as GeMSS, Gui and DualModeMS. MQsoft is an improved version of the GeMSS additional implementation submitted to the NIST post-quantum cryptography competition [oST17]. Our library permits to improve the fastest known implementations for GeMSS and Gui, as well as the signature generation of DualModeMS. The performance results are studied in Section 5. Table 1 summarizes the obtained speed-ups. For the levels of security 128 and 192 bits of Gui, we modify slightly a security parameter to improve the performance (cf. Section 5.2).

Table 1: Speed-up of GeMSS, Gui and DualModeMS (best implementation provided for the NIST submissions versus our implementation). We use a Haswell processor (ServerH).

\begin{tabular}{|c|c|c|c|c|}
\hline scheme & sec. level & key gen. & signature gen. & signature verif. \\
\hline \multirow{3}{*}{ GeMSS } & 128 & 2.8 & 1.57 & 1.98 \\
\cline { 2 - 5 } & 192 & 2.4 & 1.25 & 1.83 \\
\cline { 2 - 5 } & 256 & 2.3 & 1.23 & 1.75 \\
\hline \multirow{2}{*}{ Gui } & 128 & 13 & 1.73 & 1.74 \\
\cline { 2 - 5 } & 192 & 14 & 1.21 & 1.59 \\
\cline { 2 - 5 } & 256 & 19 & 2.5 & 1.59 \\
\hline Inner.DualModeMS & 128 & 2.2 & 1.30 & 2.1 \\
\hline DualModeMS & 128 & 1.00 & 1.32 & 1.00 \\
\hline
\end{tabular}

The structure of MQsoft is depicted in Figure 1 which summarizes the main tasks required for each cryptographic operation. The critical part of an operation is represented by a plain arrow, whereas less important operations are represented by dotted arrows.

It is clear from Figure 1, that HFE-based schemes require an efficient implementation of arithmetic in $\mathbb{F}_{2^{n}}[X]$ and so in $\mathbb{F}_{2^{n}}$. This is studied in Section 2. We have implemented stateof-the-art algorithms for arithmetic in $\mathbb{F}_{2^{n}}$ that use vectorization (sse2 and avx2) and the PCLMULQDQ instruction to improve multiplication in $\mathbb{F}_{2^{n}}$ (Section 2.2). The multiplication is computed with the schoolbook algorithm in blocks of 64 bits for $n \leq 384$ and with Karatsuba otherwise. When PCLMULQDQ is not available, MQsoft uses the multiplication in $\mathbb{F}_{2}[X]$ of the gf2x library (Section 1.7). The modular inverse is computed with the Itoh-Tsujii Multiplicative Inversion Algorithm (Section 2.5) together with multi-squaring tables (Section 2.4).

To optimize the arithmetic in $\mathbb{F}_{2^{n}}$, the choice of $n$ must be made before the compilation. This permits the specialization of the implementation. The library is flexible and allows to the choice of any $n \leq 576 . \mathbb{F}_{2^{n}}$ is built as $\mathbb{F}_{2}$ quotiented by an irreducible polynomial $f$ of degree $n$. When it is possible, we choose an irreducible trinomial for $f$ to accelerate the modular reduction (Section 2.3). The modular reduction by $f$ is vectorized for trinomials such that the degree of $f(x)-x^{n}$ is strictly less than 128 , and for the parameters of studied schemes. We have vectorized the modular reduction by a pentanomial exclusively 


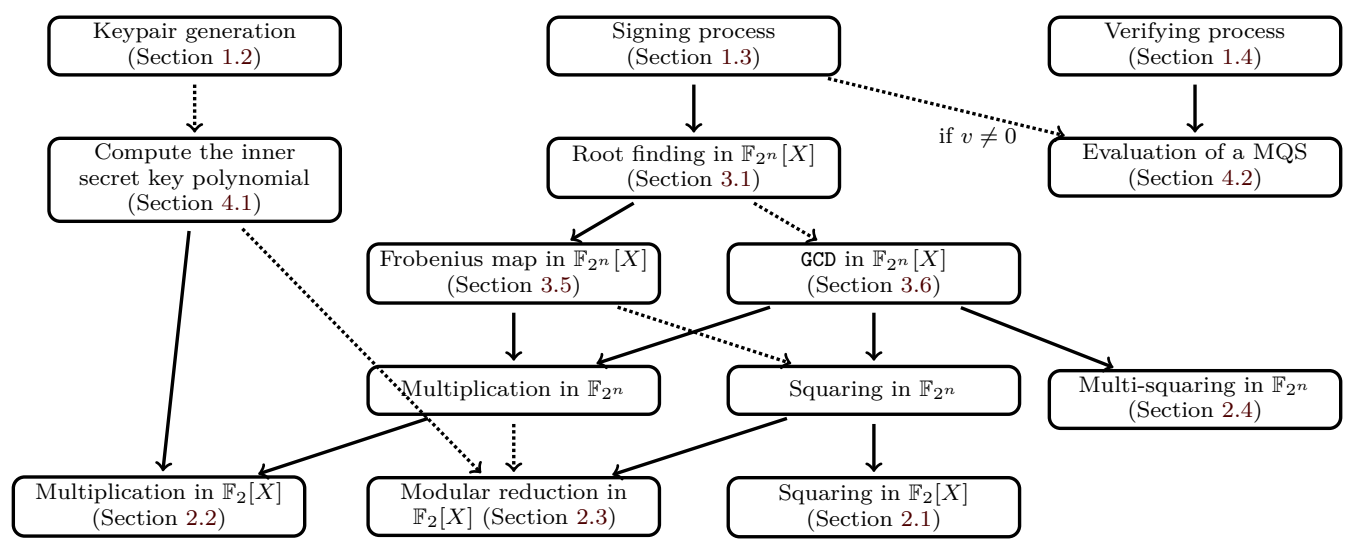

Figure 1: Dependencies between the different operations performed in MQsoft.

for $n \in\{184,312,448,544\}$, because they are the parameters of Gui and DualModeMS256. Otherwise, the modular reduction is implemented for pentanomials such that the degree of $f(x)-x^{n}$ is strictly less than 33 . For $n \leq 576,56 \%$ of the finite fields can be created with an irreducible trinomial. Our library vectorizes modular reduction for $92 \%$ of these cases. We obtain approximately a speed-up of a factor of 4 compared to the arithmetic in $\mathbb{F}_{2^{n}}$ of NTL.

In Section 4.2, the verifying process is accelerated via an efficient evaluation of multivariate quadratic systems using avx2 set instructions. We obtain new speed records for the constant-time and variable-time evaluations of binary multivariate polynomials. To do this, we have chosen to use the "monomial representation" as in $\left[\mathrm{CLP}^{+} 18\right]$. For this, we have stored multivariate quadratic systems of $m$ equations in $\mathbb{F}_{2}\left[x_{1}, \ldots, x_{n+v}\right]$ as a pair $(C, Q) \in \mathbb{F}_{2^{m}} \times \mathcal{M}_{n+v}\left(\mathbb{F}_{2^{m}}\right)$, where $Q$ is a upper triangular matrix such that $Q_{i, j}$ corresponds to the term $x_{i} x_{j}$, and $C$ is the constant term. Since the multivariate quadratic systems will be evaluated in $\mathbb{F}_{2}^{n+v}, x_{i}^{2}=x_{i}$ and so, the linear term $x_{i}$ is stored with the term $x_{i}^{2}$ of $Q$. With this representation, the evaluation in $\mathbf{x} \in \mathbb{F}_{2}^{n+v}$ is computed as $C+\mathbf{x} Q \mathbf{x}^{t}$. For 256 equations and 256 variables in $\mathbb{F}_{2}$, our variable-time evaluation is 1.38 times faster than in $\left[\mathrm{CLP}^{+} 18\right]$. To obtain this, we use unrolled loops and a specific way to extract the terms $x_{i}$. For the constant-time evaluation, we obtain a performance similar to $\left[\mathrm{CLP}^{+} 18\right]$, which targets Haswell processors. However, on Skylake processors, the evaluation can be faster by using vector instructions in a specific way, as explained in Section 4.2. This method saves a factor 1.1 on Skylake (for 256 equations and 256 variables).

The core of the signing process is to find the roots of a univariate HFE polynomial $F$ in $\mathbb{F}_{2^{n}}[X]$, which has a special structure. In particular, $F$ is in the following note:

$$
\sum_{\substack{0 \leqslant j<i<n \\ 2^{i}+2^{j} \leqslant D}} A_{i, j} X^{2^{i}+2^{j}}+\sum_{\substack{0 \leqslant i<n \\ 2^{i} \leqslant D}} B_{i} X^{2^{i}}+C \in \mathbb{F}_{2^{n}}[X] .
$$

Our goal is to exploit this structure to accelerate the root finding. We address this question in Section 3. We have been able to tweak Berlekamp's algorithm [vzGG13, Algorithm $14.15]$ to take advantage of the sparse structure of $F$.

When $D>n$, the computation of $X^{2^{n}} \bmod F$ is done with the repeated squaring algorithm [vzGG13, Algorithm 4.8]. The core of this algorithm is to compute the modular reduction of the square of an element $B \in \mathbb{F}_{2^{n}}[X]$ by $F$. The classical Euclidean division of $B^{2}$ 
by $F$ requires to compute $B^{2}-Q F$, where $Q$ is the quotient of this division. With a naive implementation, the multiplication of $Q$ by $F$ costs $O\left(D^{2}\right)$ field multiplications. Using a sparse representation of $F$, the multiplication costs only $O\left(D \log _{2}(D)^{2}\right)$ field multiplications.

So, with a sparse polynomial, the computation of the roots is faster. This suggests to consider sparse HFE polynomials. In Theorem 1, we prove that making $F$ more sparse improves the complexity. Because $F$ is a part of the secret-key, the nature of this change requires a new analysis of security. We observe in practice that removing a small number of odd degree terms appears not to affect the security. However, the security of this method must be studied in depth. With Theorem 1, we can save $43.75 \%$ of the computations we would have done by removing only three terms having an odd degree in $F$. The general idea to make $F$ more sparse has already been proposed in HFEBoost ${ }^{1}$, but independently of this, the proof of Theorem 1 makes explicit a method to improve the complexity. It has the advantage of being in constant-time because the useless computations are known and so can be avoided.

Theorem 1. Let $H$ be a HFE polynomial of degree $D$ in $\mathbb{F}_{2^{n}}[X]$ where the $k$-th terms of highest odd degree have been removed $\left(k \in \llbracket 0,\left\lceil\log _{2}(D)\right\rceil-1 \rrbracket\right)$, and let $A \in \mathbb{F}_{2^{n}}[X]$ be a square of degree at most $2 D-2$. If $D$ is even, then the computation of the classical Euclidean division (Algorithm 8) of $A$ by $H$ can be accelerated by a factor $(D-1) /\left(\frac{D}{2}+\right.$ $\left.\left\lfloor 2^{\left\lceil\log _{2}(D)\right\rceil-k-2}\right\rfloor\right)$.

When $D<n$, the strategy of $\left[\mathrm{PCY}^{+} 15\right]$ becomes more efficient for computing the Frobenius map. The idea is to compute a table of $X^{2 i} \bmod F$ to accelerate the modular reduction. Thus, the squaring modulo $F$ is computed by multiplying its $i$-th coefficient by the element $X^{2 i} \bmod F$ from the table for $i \in \llbracket 0, D-1 \rrbracket$. The authors of $\left[\mathrm{PCY}^{+} 15\right]$ also suggest to do several squarings in one step, with multi-squaring tables. In Section 3.5, we make an explicit strategy for doing this efficiently (by exploiting the HFE structure of $F$ when it is possible), and how to choose the number of squarings to do before the modular reduction.

The performance of both strategies described above depends on the required number of field multiplications. In Section 3.5, we compute accurately the number of multiplications of each method, in order to choose the best strategy as a function of the parameters.

\section{Preliminaries}

We briefly recall here the principle of a signature scheme based on HFEv- [KPG99]. The public-key in HFEv- is given by a set of $m$ quadratic equations in $\mathbb{F}_{2}\left[x_{1}, \ldots, x_{n+v}\right]$. These equations are derived from a single polynomial $F \in \mathbb{F}_{2^{n}}[X]$ (Section 1.2). Verification requires to evaluate the public polynomials (Section 1.4). We need to compute the roots of $F$ in order to generate a signature (Section 1.3). From now, we always assume that the base field is $\mathbb{F}_{2}$. In Section 1.5, we introduce the specificities of GeMSS and Gui which are two submissions to the NIST PQC process based on HFEv-.

\subsection{Main Parameters}

The main parameters involved are:

- $D$, a positive integer that corresponds to the degree of a secret polynomial. $D$ is such that $D=2^{i}$ for $i \geq 0$, or $D=2^{i}+2^{j}$ for $i \neq j$, and $i, j \geq 0$,

- $m$, number of equations in the public-key,

\footnotetext{
${ }^{1}$ https://www-polsys.lip6.fr/Links/hfeboost.html
} 
- $n$, the degree of a field extension of $\mathbb{F}_{2}$,

- $v$, the number of vinegar variables,

- $\Delta$, the number of minus (the number of equations in the public-key is such that is $m=n-\Delta)$,

- nb_ite $\geq 1$, number of iterations in the verification and signature processes.

\subsection{Keypair Generation}

Secret-key. It is composed by a couple of invertible matrices ${ }^{2}(\mathbf{S}, \mathbf{T}) \in \mathrm{GL}_{n+v}\left(\mathbb{F}_{2}\right) \times$ $\mathrm{GL}_{n}\left(\mathbb{F}_{2}\right)$ and a polynomial $F \in \mathbb{F}_{2^{n}}\left[X, v_{1}, \ldots, v_{v}\right]$ with the following structure:

$$
\sum_{\substack{0 \leqslant j<i<n \\ 2^{i}+2^{j} \leqslant D}} A_{i, j} X^{2^{i}+2^{j}}+\sum_{\substack{0 \leqslant i<n \\ 2^{i} \leqslant D}} \beta_{i}\left(v_{1}, \ldots, v_{v}\right) X^{2^{i}}+\gamma\left(v_{1}, \ldots, v_{v}\right),
$$

where $A_{i, j} \in \mathbb{F}_{2^{n}}, \forall i, j, 0 \leqslant j<i<n$, each $\beta_{i}: \mathbb{F}_{2}^{v} \rightarrow \mathbb{F}_{2^{n}}$ is linear and $\gamma\left(v_{1}, \ldots, v_{v}\right)$ : $\mathbb{F}_{2}^{v} \rightarrow \mathbb{F}_{2^{n}}$ is quadratic. The variables $v_{1}, \ldots, v_{v}$ are called the vinegar variables. We shall say that a polynomial $F \in \mathbb{F}_{2^{n}}\left[X, v_{1}, \ldots, v_{v}\right]$ with the form of (1) has a HFEv-shape. The particularity of a polynomial $F\left(X, v_{1}, \ldots, v_{v}\right)$ with HFEv-shape is that for any specialization of the vinegar variables the polynomial $F$ becomes a HFE polynomial [Pat96], i.e. univariate polynomial of the following form:

$$
\sum_{\substack{0 \leqslant j<i<n \\ 2^{i}+2^{j} \leqslant D}} A_{i, j} X^{2^{i}+2^{j}}+\sum_{\substack{0 \leqslant i<n \\ 2^{i} \leqslant D}} B_{i} X^{2^{i}}+C \in \mathbb{F}_{2^{n}}[X],
$$

with $A_{i, j}, B_{i}, C \in \mathbb{F}_{2^{n}}, \forall i, j, 0 \leqslant j<i<n$. By abuse of notation, we will refer to $D$ as the degree of the HFEv polynomial.

The special structure of (1) is chosen such that its multivariate representation over the base field $\mathbb{F}_{2}$ is composed by quadratic polynomials in $\mathbb{F}_{2}\left[x_{1}, \ldots, x_{n+v}\right]$. This is due to the special exponents chosen in $X$ that have all a binary decomposition of Hamming weight at most 2 .

Let $\theta=\left(\theta_{1}, \ldots, \theta_{n}\right) \in\left(\mathbb{F}_{2^{n}}\right)^{n}$ be a basis of $\mathbb{F}_{2^{n}}$ over $\mathbb{F}_{2}$. We set

$$
\varphi: E=\sum_{k=1}^{n} e_{k} \cdot \theta_{k} \in \mathbb{F}_{2^{n}} \longrightarrow \varphi(E)=\left(e_{1}, \ldots, e_{n}\right) \in \mathbb{F}_{2}^{n} .
$$

We can now define a set of multivariate polynomials $\mathbf{f}=\left(f_{1}, \ldots, f_{n}\right) \in \mathbb{F}_{2}\left[x_{1}, \ldots, x_{n+v}\right]^{n}$ derived from a HFEv polynomial $F \in \mathbb{F}_{2^{n}}\left[X, v_{1}, \ldots, v_{v}\right]$ by:

$$
F\left(\sum_{k=1}^{n} \theta_{k} x_{k}, v_{1}, \ldots, v_{v}\right)=\sum_{k=1}^{n} \theta_{k} f_{k} .
$$

For easing the notations, we now identify the vinegar variables $\left(v_{1}, \ldots, v_{v}\right)=\left(x_{n+1}, \ldots, x_{n+v}\right)$. Besides, we shall say that the polynomials $f_{1}, \ldots, f_{n} \in \mathbb{F}_{2}\left[x_{1}, \ldots, x_{n+v}\right]$ are the components of $F$ over $\mathbb{F}_{2}$.

In the implementation, we compute the components of $F$ by using directly Equation (3). We replace $X$ by $\sum_{k=1}^{n} \theta_{k} x_{k}, v_{1}, \ldots, v_{v}$ in the expression of $F$, then we use a rearrangement of terms which minimizes the number of multiplications. This is detailed in Section 4.1.

\footnotetext{
${ }^{2}$ In full generality, one can have affine transformations. We choose linear transformations for the sake of simplicity.
} 
Public-key. It is given by a set of $m$ quadratic square-free non-linear polynomials $\mathbf{p}=$ $\left(p_{1}, \ldots, p_{m}\right) \in \mathbb{F}_{2}\left[x_{1}, \ldots, x_{n+v}\right]^{m}$. It is obtained from the secret-key by taking the first $m=n-\Delta$ polynomials of:

$$
\left(f_{1}\left(\left(x_{1}, \ldots, x_{n+v}\right) \mathbf{S}\right), \ldots, f_{n}\left(\left(x_{1}, \ldots, x_{n+v}\right) \mathbf{S}\right)\right) \mathbf{T},
$$

and reducing it modulo the field equations, i.e. modulo $\left\langle x_{1}^{2}-x_{1}, \ldots, x_{n+v}^{2}-x_{n+v}\right\rangle$.

We summarize the public-key/secret-key generation in Algorithm 1. In practice, we merge the steps 6 and 7 by removing the $\Delta$ last columns of $\mathbf{T}$ during the vector matrix product.

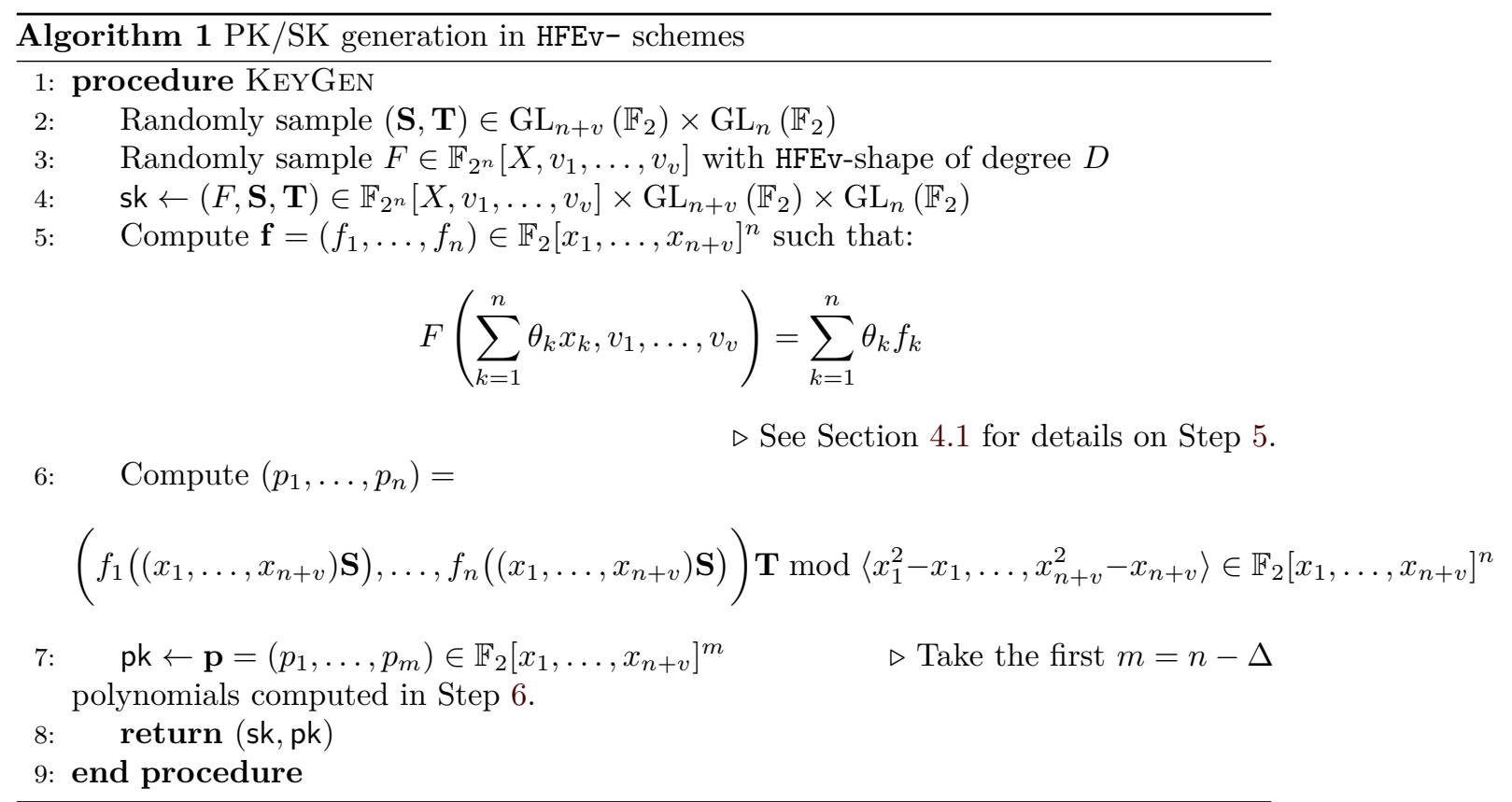

\subsection{Signing process}

The main step of the signature process requires to invert the public-key, that is to say solving:

$$
p_{1}\left(x_{1}, \ldots, x_{n+v}\right)-d_{1}=0, \ldots, p_{m}\left(x_{1}, \ldots, x_{n+v}\right)-d_{m}=0 .
$$

for $\mathbf{d}=\left(d_{1}, \ldots, d_{m}\right) \in \mathbb{F}_{2}^{m}$.

To do so, we randomly sample $\mathbf{r}=\left(r_{1}, \ldots, r_{\Delta}\right) \in \mathbb{F}_{2}^{\Delta}$ and append it to $\mathbf{d}$. This gives $\mathbf{d}^{\prime}=(\mathbf{d}, \mathbf{r}) \in \mathbb{F}_{2}^{n}$. We compute then $D^{\prime}=\varphi^{-1}\left(\mathbf{d}^{\prime} \times \mathbf{T}^{-1}\right) \in \mathbb{F}_{2^{n}}$ and try to find a root $\left(Z, z_{1}, \ldots, z_{v}\right) \in \mathbb{F}_{2^{n}} \times \mathbb{F}_{2}^{v}$ of the multivariate equation:

$$
F\left(Z, z_{1}, \ldots, z_{v}\right)-D^{\prime}=0
$$

To solve this equation, we take advantage of the special HFEv-shape. That is why, we randomly sample $\mathbf{v} \in \mathbb{F}_{2}^{v}$ and consider the univariate polynomial $F(X, \mathbf{v}) \in \mathbb{F}_{2^{n}}[X]$. This yields a HFE polynomial according to Section 1.2. We then find the roots of the univariate equation:

$$
F(X, \mathbf{v})-D^{\prime}=0 .
$$


If there is a root $Z \in \mathbb{F}_{2^{n}}$, we return $(\varphi(Z), \mathbf{v}) \times \mathbf{S}^{-1} \in \mathbb{F}_{2}^{n+v}$.

A core part of the signature generation is then the computation of the roots of $F_{D^{\prime}}(X)=$ $F(X, \mathbf{v})-D^{\prime}$. To this end, we use the Berlekamp algorithm as described in [vzGG13, Algorithm 14.15], which requires mainly to compute:

$$
\operatorname{GCD}\left(X^{2^{n}}-X \bmod F_{D^{\prime}}, F_{D^{\prime}}\right)
$$

We provide in Section 3.1 the best methods to compute $X^{2^{n}} \bmod F_{D^{\prime}}$ and the GCD (both in function of $n$ and $D$ ).

We can now present a way to define the inversion function (Algorithm 2):



The signing algorithm in GeMSS and Gui is an iterative process. The basic idea is to call $\mathrm{Inv}_{\mathbf{p}} \mathrm{nb}$ _ite times. More precisely, the signing process is in the following algorithm:

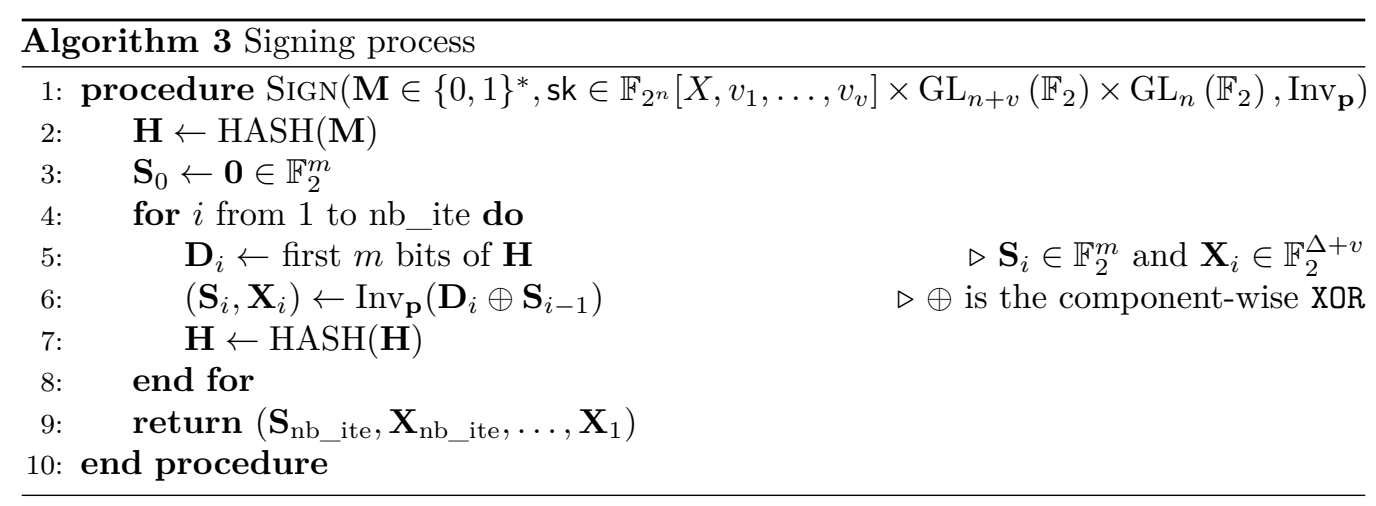

nb_ite is a parameter that can be easily computed from $m$ and the level of security.

\subsection{Verifying process}

Naturally, the verifying process is also iterative as shows in Algorithm 4. The main part of this process is still to evaluate the public-key. We describe how to implement this step efficiently in Section 4.2 . 




\subsection{GeMSS and Gui}

GeMSS and Gui are essentially based on the same principle. They still differ in the choice of parameters.

Choice of parameters. Table 26 summarizes the performance of the best implementations of GeMSS and Gui provided for the NIST submissions, in function of the parameters. For GeMSS, we consider the additional implementation. For Gui, we take the PCLMULQDQ additional implementation. Gui is implemented with the modified algorithms to achieve EUF-CMA security property $\left[\mathrm{CDP}^{+} 17\right.$, Section 1.6]. It is not the case for the GeMSS implementation. For this reason, we have modified the Gui additional implementation. This implementation provides the cryptographic operations of Gui with and without the EUF-CMA security property. We just replace the algorithms by their version without this property. Compared to the original implementation, this implies mainly a speed-up during the signature generation.

In Gui, the parameters are chosen to minimize the time of signing and verifying a signature. To do it, small values of $D$ are chosen, and larger values of $m$ are used. This choice implies to increase $n$, and so the size of the public-key. In GeMSS, it is the opposite. The goal is to minimize the size of the public-key. This leads to smaller values of $n$. This choice implies to take larger values of $D$.

As soon as $m$ is fixed, the number of iterations required can be derived. In fact, the original parameters of Gui do not always provide the claimed security. The attack described in [Cou03, Theorem 6.2.1] has a complexity $O\left(2^{m \frac{\mathrm{nb} \text { ite }}{\mathrm{nb}-\mathrm{ite}+1}}\right)$. So, with $m=168$ and nb_ite $=2$, the original parameters of Gui-184 provide a security of only 112 bits. This problem has been mentioned in NIST's pqc-forum mailing list by W. Beullens the 04/27/2018. The Gui designers have answered the 06/15/2018, and choose to set the parameter nb_ite to 3 . However, this provides only 126 bits of security. To reach 128 bits of security, we should set nb_ite to 4 (or to set $m$ to 171). For completeness, we have measured Gui-184 for these three values of nb_ite. The modification slows down a factor $\frac{\mathrm{nb} \text { ite }}{2}$ the signature generation and the verifying process of Gui-184.

To find a root of $\boldsymbol{F}(\boldsymbol{X}, \mathbf{v})-\boldsymbol{D}^{\prime}$. During the signature generation, $F(X, \mathbf{v})-D^{\prime}$ cannot have solution. In GeMSS, $\mathbf{r}$ and $\mathbf{v}$ are changed while $F(X, \mathbf{v})-D^{\prime}$ does not have roots (cf. Algorithm 2). Then, when there are roots, one is deterministically chosen for fixed $D^{\prime}$, by using the SHA-3 of $D^{\prime}$ as a randombytes generator. In Gui, $\mathbf{v}$ is changed while 
$F(X, \mathbf{v})-D^{\prime}$ does not have a unique root.

In practice, $\mathbf{d}$ is generated from the hash of a document. To do it, GeMSS uses SHA-3, whereas Gui uses SHA-2. The output size of the hash functions is the double of the level of security. In MQsoft, we have chosen the SHA-3 function from the Keccak Code Package [GBA13] and the SHA-2 function from OpenSSL.

\subsection{Data Structure}

We describe here the data structure used to store elements of $\mathbb{F}_{2^{n}}$ and $\mathbb{F}_{2^{n}}[X]$. This representation is crucial for the efficiency of our implementation. This is especially true for binary fields since operations in $\mathbb{F}_{2}$ can be naturally vectorized. The arithmetic in $\mathbb{F}_{2^{n}}[X]$ is used during the root finding of a HFE polynomial. To be efficient, it is important to distinguish dense polynomials which appear during the computation of the Frobenius map and the GCD, from a HFE polynomial which is used to reduce $X^{2^{n}}-X$. We can notice that the HFE polynomial is sparse since it has only $K=O\left(\log _{2}(D)^{2}\right)$ non-zero coefficients.

Representation of elements in $\mathbb{F}_{2^{n}}$. The field $\mathbb{F}_{2^{n}}$ is defined as $\frac{\mathbb{F}_{2}[X]}{f(x)}$ with $f(x)$ being an irreducible polynomial of degree $n$ in $\mathbb{F}_{2}[x]$. We have chosen the polynomial basis [HMV03]. An element of $\mathbb{F}_{2^{n}}$ is represented by a polynomial in $\mathbb{F}_{2}[x]$ of degree at most $n-1$. The coefficients are stored as a vector of bits, requiring $\left\lceil\frac{n}{w}\right\rceil$ words, where $w$ is the word size (in bits). The $j$-th bit of the $i$-th word is the coefficient of the term of degree $w i+j$, for $i \in \llbracket 0,\left\lceil\frac{n}{w}\right\rceil-1 \rrbracket$ and $j \in \llbracket 0, w-1 \rrbracket$. It is setting to zero when $(w i+j) \geq n$.

Example 1. Let $w=64$ and $P=x^{36}+x^{4} \in \mathbb{F}_{2^{40}}$. To simplify the notations, we represent vectors of bits as 64-bit integers. $P$ is stored as $0 \times 0000001000000010$. In particular, the bits from 37 to 63 are setting to zero.

Representation of dense polynomials in $\mathbb{F}_{2^{n}}[\boldsymbol{X}]$. An element of $\mathbb{F}_{2^{n}}[X]$ is represented by its degree $\delta$ and a vector of $(\delta+1)$ coefficients. The coefficients are stored from lower to higher degree of the corresponding terms in a buffer. The degree is stored in a local variable, excepted for the implementation of the fast GCD in Section 3.6, because it requires matrices in $\mathcal{M}_{2}\left(\mathbb{F}_{2^{n}}[X]\right)$. In this case, we use a $\mathrm{C}$ structure to store the degree and the pointer toward the coefficients buffer.

Example 2. Let $P=X^{8}+\alpha X^{7}+(\alpha+1) X^{6}+X^{5}+\alpha X \in \mathbb{F}_{4}[X]$ and $f(\alpha)=\alpha^{2}+\alpha+1$. $P$ is stored as $8,(0, \alpha, 0,0,0,1, \alpha+1, \alpha, 1)$.

Representation of HFE polynomials in $\mathbb{F}_{\mathbf{2}^{n}}[\boldsymbol{X}]$. In HFEv scheme, the HFEv polynomial is a part of the secret-key. During the signature generation (cf. Section 1.3), the vinegar variables of the HFEv polynomial are evaluated to obtain a HFE polynomial. Its degree $D$ is a parameter of security and is assumed to be known. It is defined by the $\mathrm{C}$ directive \#define. A HFE polynomial in $\mathbb{F}_{2^{n}}[X]$ is represented as a vector of coefficients where only terms $X^{0}, X^{2^{i}}$ and $X^{2^{i}+2^{j}}$ are stored. It is chosen monic and so the leading term is not stored. If $P$ is in $\mathbb{F}_{2^{n}}[X]$, we denote by $P_{\mathrm{HFE}}$ its HFE representation.

Example 3. Let $P=X^{16}+\alpha X^{12}+(\alpha+1) X^{10}+\alpha X \in \mathbb{F}_{4}[X]$ and $f(\alpha)=\alpha^{2}+\alpha+1$. $P_{\mathrm{HFE}}$ is stored as $(0, \alpha, 0,0,0,0,0,0,0, \alpha+1, \alpha)$. Only the coefficients of terms with a degree in $\{0,1,2,3,4,5,6,8,9,10,12\}$ are stored.

\subsection{Experimental Platform and Benchmarked Libraries}

Tables 2 and 3 summarize the main informations about the platform used in the experimental measurements. LaptopS is used for all measurements, excepted in Section 4.2 
Table 2: Processors.

\begin{tabular}{|l|l|l|l|l|}
\hline Computer & Processor & Frequency & Max freq. & Architecture \\
\hline LaptopS & Intel(R) Core(TM) i7-6600U CPU & $2.60 \mathrm{GHz}$ & $3.40 \mathrm{GHz}$ & Skylake \\
\hline ServerH & Intel(R) Xeon(R) CPU E3-1275 v3 & $3.50 \mathrm{GHz}$ & $3.90 \mathrm{GHz}$ & Haswell \\
\hline DesktopH & Intel(R) Core(TM) i7-4790 CPU & $3.60 \mathrm{GHz}$ & $4.00 \mathrm{GHz}$ & Haswell \\
\hline DesktopS & Intel(R) Core(TM) i7-6700 CPU & $3.40 \mathrm{GHz}$ & $4.00 \mathrm{GHz}$ & Skylake \\
\hline
\end{tabular}

Table 3: OS and Memory.

\begin{tabular}{|l|l|l|l|l|l|l|}
\hline Computer & OS & RAM & L1d & L1i & L2 & L3 \\
\hline LaptopS & Ubuntu 16.04.5 LTS & $32 \mathrm{~GB}$ & $32 \mathrm{~KB}$ & $32 \mathrm{~KB}$ & $256 \mathrm{~KB}$ & $4096 \mathrm{~KB}$ \\
\cline { 1 - 2 } ServerH & CentOS Linux 7 (Core) & & & & & $8192 \mathrm{~KB}$ \\
\cline { 1 - 2 } DesktopH & Debian GNU/Linux 9 & $16 \mathrm{~GB}$ & & & & \\
\cline { 1 - 2 } & & & & & & \\
\hline
\end{tabular}

and in Section 5. For this latter, we present the performance of MQsoft in function of the processor. Our implementation targets Intel 64-bit processors that support the PCLMULQDQ instruction. This allows to improve the performance of multiplication in $\mathbb{F}_{2^{n}}$ (cf. Section 2.1 and 2.2). We also take advantage of the sse2, ssse3 and avx2 instruction sets to speed-up the implementation of arithmetic in $\mathbb{F}_{2^{n}}$ (Section 2), the vector matrix product in $\mathbb{F}_{2}$ during the keypair generation (Section 5.4), and the evaluation of the public-key during the verifying process (Section 4.2). We explain here the main vector instructions ${ }^{3}$ that MQsoft exploits:

- PCLMULQDQ: this instruction computes the product of two binary polynomials such that their degree is strictly less than 64 .

- PSHUFB: this instruction from ssse3 takes 16 indexes on 4 bits, and searches the corresponding 8-bit elements in a table of size 128 bits.

- VPSHUFB: this instruction from avx2 performs two times PSHUFB.

- PSLLDQ and PSRLDQ: these instructions from sse2 computes respectively the left and right shift of a 128-bit register by a multiple of 8 bits.

- PALIGNR: this instruction from ssse3 concatenates two registers 128 bits, shifts the concatenation at right by a multiple of 8 bits, then return the 128 lower bits of the result.

- VPBROADCASTQ: this instruction from avx duplicates four times a 64-bit integer on a 256-bit register.

- VPERMQ: this instruction from avx2 permutes the 64-bit parts of a 256-bit register. In particular, it can duplicate one 64-bit part four times.

- VPMASKMOVQ: this instruction from avx2 loads four contiguous 64-bit integers from a buffer, then applies a mask which permits to set to zero 64-bit parts.

- POPCNT: this instruction counts the number of bits set to 1 in 64-bit integers. It is used to speed-up the dot product of vectors in $\mathbb{F}_{2}$.

\footnotetext{
${ }^{3}$ For more informations, we refer to the Intel Intrinsics Guide (https://software.intel.com/sites/ landingpage/IntrinsicsGuide/\#).
} 
We benchmarked our software toolkit against the following softwares or libraries.

- Magma ([BCM94], version 2.23-6). Magma is a computer algebra software, well-known to be very efficient over finite fields.

- NTL ([Sho03], version 10.5.0), installed with GMP ([Ga02], version 6.1.2). NTL is one of the best library for number theory. It is implemented in $\mathrm{C}++$.

- $\operatorname{gf} 2 \mathrm{x}([\mathrm{BGTZ08}]$,version 1.2). $\mathrm{gf} 2 \mathrm{x}$ is a C implementation of the state-of-the-art multiplications in $\mathbb{F}_{2}[X]$. The multiplication algorithm depends on the degree of the operands and the vector instructions set available. When PCLMULQDQ is not available, MQsoft uses the gf $2 \mathrm{x}$ multiplication.

Magma is running with magma. avx64.dyn. exe to take advantage of vector instructions. In our tests, the avx64 version optimizes mainly the performance of multiplication in binary fields.

The measurements used one core of the CPU, and the $\mathrm{C}$ code was compiled with gcc -04 -mavx2 -mpclmul -mpopcnt-funroll-loops. We use the version 6.4.0 of gcc. Turbo Boost and Enhanced Intel Speedstep Technology are disabled to have more accurate measurements, excepted when we use DesktopH and DesktopS. In practice, Turbo Boost generates a speed-up of 1.2 on LaptopS and 1.1 on ServerH.

\section{Efficient Arithmetic in $\mathbb{F}_{2^{n}}$}

Arithmetic in $\mathbb{F}_{2^{n}}$ is the core of the signature generation (Section 1.3) and the computation of $\mathbf{f}$ during the keypair generation (Section 1.2). The main involved parameters (Section 1.1) are $n$ the degree of the field extension, $v$ the number of vinegar variables, $D$ the degree of the HFEv polynomial and nb_ite a constant between two and four in GeMSS and Gui. In Section 4.1, we explain how to generate efficiently the inner secret polynomials of $\mathbf{f}$ (Equation (3)). This requires $O\left(n \log _{2}(D)\right)$ squarings in $\mathbb{F}_{2^{n}}, O\left(n \log _{2}(D)\left(n+v+\log _{2}(D)\right)\right.$ multiplications in $\mathbb{F}_{2}[X]$ and $O\left(n\left(n+v+\log _{2}(D)\right)\right)$ modular reductions. In Section 3, the signature generation requires $O\left(\right.$ nb_ite $\left.\times\left(n D \log _{2}(D)^{2}+D^{2}\right)\right)$ field multiplications, $O($ nb_ite $\times n D)$ field squarings and $O($ nb_ite $\times D)$ field inversions.

We use the polynomial representation defined in Section 1.6. It is the most efficient representation when PCLMULQDQ is available $\left[\mathrm{TFA}^{+} 11\right]$. To compute the square (respectively the multiplication) of $B$ in $\mathbb{F}_{2^{n}}$, we choose to compute the square (respectively the multiplication) of $B$ in $\mathbb{F}_{2}[X]$ before to reduce the reduction by the univariate polynomial defining the extension.

Table 4: Performance of the PCLMULQDQ instruction in function of the architecture, as presented in the Intel Intrinsics Guide.

\begin{tabular}{|l|l|l|l|l|}
\hline Architecture & Skylake & Broadwell & Haswell & Ivy Bridge \\
\hline Latency & 7 & 5 & 7 & 14 \\
\hline Throughput (CPI) & 1 & 1 & 2 & 8 \\
\hline
\end{tabular}

Table 4 presents the cost of PCLMULQDQ in function of the architecture. The choice of the best algorithm of multiplication in $\mathbb{F}_{2^{n}}$ depends on the processor. Our choices target the Skylake processors, which use only one CPI (cycle per instruction).

\subsection{Squaring in $\mathbb{F}_{2^{n}}$}

Squaring is used during the root finding algorithm (Section 3) which is the core of the signature generation (Section 1.3). It is also used during the so called Itoh-Tsujii algorithm 
[IT88] which computes the modular inverse in $\mathbb{F}_{2^{n}}^{\times}$(Section 2.5).

In binary fields, the squaring of $B=\sum_{i=0}^{n-1} \alpha_{i} x^{i} \in \mathbb{F}_{2^{n}}$ can be performed in linear time [LN96]. The linearity of the Frobenius endomorphism implies that $B^{2}=\sum_{i=0}^{n-1} \alpha_{i} x^{2 i}$. Since we have stored $B$ as a vector of bits, squaring is the equivalent to insert a null bit between each bit of $B$.

Example 4. Let $B=x^{3}+x^{2}+1 \in \mathbb{F}_{2^{4}}$. $B$ is stored as the binary integer 1101. Its square is $B^{2}=x^{6}+x^{4}+1$, which is represented as 1010001 .

To compute the square of a $n$-bit element, we divide it into words of 64 bits. For each one, the PCLMULQDQ instruction computes directly the binary polynomial multiplication of the 64-bit element by itself. This method requires $\left\lceil\frac{n}{64}\right\rceil$ calls to PCLMULQDQ.

We have compared this method to table lookups of square [ALH10a]. We have implemented Algorithm 1 of [ALH10a] which uses sse2 instructions and PSHUFB from ssse3, and an avx2 version that uses the VPSHUFB instruction. The PSHUFB instruction performs the search of the square of 16 elements on 4 bits in a table in constant-time, and the VPSHUFB instruction performs two times PSHUFB. On Skylake, both are less efficient than the PCLMULQDQ instruction, whereas on Haswell, we observe the opposite behavior because PCLMULQDQ is slower (Table 4).

Table 5 summarizes the performance of squaring functions which are proposed in our library. The experimental process consists to compute the square of elements from a small buffer, then to measure the average cost of one operation. In order to compute the square with table lookups, the PSHUFB instructions must be used by pair: one computes the square of lower 4-bit elements of each byte, whereas the second is used for the higher 4-bit elements. For this reason, the squaring performance using PSHUFB depends only on $\left\lceil\frac{n}{128}\right\rceil$. For the squaring using PCLMULQDQ, the implementation for $\left\lceil\frac{n}{64}\right\rceil$ equals to 3 is slower than for $\left\lceil\frac{n}{64}\right\rceil$ equals to 4 . Indeed, 3 is odd and the 128 -bit load and store instructions are less efficient when they are used to load and store a 64-bit element. The best squaring is the one using the PCLMULQDQ instruction: on the Skylake processors, it costs only one cycle of throughput, but seven cycles of latency (Table 4). However, the latency can be used to do other instructions, which improves the performance.

Table 5: Number of cycles for computing the square of an element of $\mathbb{F}_{2}[X]$ of degree $n-1$, with MQsoft. We use a Skylake processor (LaptopS).

\begin{tabular}{|l|l|l|l|}
\hline \multirow{2}{*}{$\left\lceil\frac{n}{64}\right\rceil$} & \multicolumn{3}{|c|}{ Squaring } \\
\cline { 2 - 4 } & PSHUFB & VPSHUFB & PCLMULQDQ \\
\hline 1 & 5.6 & $\times$ & $\mathbf{2 . 2}$ \\
\hline 2 & 5.7 & $\times$ & $\mathbf{4 . 6}$ \\
\hline 3 & 8.9 & $\times$ & $\mathbf{6 . 6}$ \\
\hline 4 & 9.0 & 7.8 & $\mathbf{5 . 8}$ \\
\hline 5 & 13.2 & $\times$ & $\mathbf{6 . 9}$ \\
\hline 6 & 13.2 & $\times$ & $\mathbf{7 . 9}$ \\
\hline 7 & 17.5 & $\times$ & $\mathbf{9 . 2}$ \\
\hline 8 & 17.5 & 12.2 & $\mathbf{1 0 . 5}$ \\
\hline 9 & 21.9 & $\times$ & $\mathbf{1 1 . 5}$ \\
\hline
\end{tabular}

\subsection{Multiplication in $\mathbb{F}_{2^{n}}$}

The multiplication of two distinct elements in $\mathbb{F}_{2^{n}}$ is a central operation involved in the keypair generation and the signing process. In MQsoft, we adapt the multiplication algo- 
rithm in function of $n$. We target the Skylake processors.

When $n \leq 384$, we use a schoolbook multiplication by blocks of 64 bits. We use the PCLMULQDQ instruction for multiplying each block. Then, $\left\lceil\frac{n}{64}\right\rceil^{2}$ calls to PCLMULQDQ are required. This method is naturally in constant-time. Our implementation uses PCLMULQDQ which implies to use sse2 instructions. We use also the PALIGNR instruction from ssse 3 to improve the implementation. This instruction concatenates two 128-bit registers and permits to extract 128 bits from the result. We use it to align on 128 bits the results of the multiplications.

When $385 \leq n \leq 576$, an element of $\mathbb{F}_{2^{n}}$ requires 7,8 or 9 words, and Karatsuba multiplication [vzGG13, section 8.1] becomes faster than the schoolbook method. We split each input in two: the low bits create a 255 degree polynomial, and the remaining bits create a $n-257$ degree polynomial. Thus, the Karatsuba algorithm requires one multiplication of 255 degree polynomials, one multiplication of $n-257$ degree polynomials and one multiplication of $\max (255, n-257)$ degree polynomials. These multiplications are computed with the schoolbook multiplication, requiring $16+\left\lceil\frac{n-256}{64}\right\rceil^{2}+\max \left(16,\left\lceil\frac{n-256}{64}\right\rceil^{2}\right)$ calls to PCLMULQDQ.

The trade-off between the schoolbook multiplication and Karatsuba depends on the performance of PCLMULQDQ (Table 4). For the Skylake processors, this instruction costs one CPI, which makes schoolbook multiplication more efficient for $n \leq 384$. For the Haswell processors, PCLMULQDQ costs two CPI. This decreases the trade-off because each call to PCLMULQDQ is more penalizing. When $\left\lceil\frac{n}{64}\right\rceil$ is equals to 3 , we remark that the Karatsuba multiplication in $\mathbb{F}_{2^{n}}$ is already faster on Haswell. In practice, we have compared the schoolbook multiplication to the three-term Karatsuba-like formulae described in [Mon05, Equation 3 with $C=0$ ]. The latter is slightly faster and requires only 6 calls to PCLMULQDQ.

Table 6 compares our multiplication with gf $2 x$. As in Section 2.1, we measure the average cost to multiply elements from a small buffer. The multiplication of $g f 2 x$ is sometimes abnormally slow. This probably dues to the fact that the implementation uses vector and no vector instructions in the same function, which penalizes it. This is probably the first reason to explain that our multiplication is faster. The second reason is that $\mathrm{gf} 2 \mathrm{x}$ uses Karatsuba, which is slower than schoolbook multiplication for $n \leq 384$ on Skylake. We have also remarked that installing NTL with $\mathrm{gf} 2 \mathrm{x}$ decreases slightly the performance. For this reason, NTL is not installed with $\mathrm{gf} 2 \mathrm{x}$ on our experimental platform.

Table 6: Number of cycles to multiply two elements of $\mathbb{F}_{2}[X]$ of degree $n-1$. We use a Skylake processor (LaptopS).

\begin{tabular}{|l|l|l|}
\hline$\left[\frac{n}{64}\right]$ & gf2x & MQsoft \\
\hline 1 & $\mathbf{3 . 4}$ & $\mathbf{3 . 4}$ \\
\hline 2 & 7.7 & $\mathbf{6 . 8}$ \\
\hline 3 & 37.0 & $\mathbf{1 5 . 5}$ \\
\hline 4 & 23.1 & $\mathbf{2 1 . 9}$ \\
\hline 5 & 47.0 & $\mathbf{3 4 . 9}$ \\
\hline 6 & 54.3 & $\mathbf{4 5 . 7}$ \\
\hline 7 & 142.2 & $\mathbf{5 9 . 2}$ \\
\hline 8 & 91.1 & $\mathbf{6 5 . 5}$ \\
\hline 9 & 131.8 & $\mathbf{9 3 . 3}$ \\
\hline
\end{tabular}




\subsection{Modular Reduction and Field Operation in $\mathbb{F}_{2^{n}}$}

In this section, we want to reduce $R=\sum_{i=0}^{2 n-2} r_{i} x^{i}$ the result of the previous multiplication/squaring in $\mathbb{F}_{2^{n}}$. The choice of the irreducible polynomial $f$ defining $\mathbb{F}_{2^{n}}$ (Section 1.6) is important for the modular reduction: it is faster when $f$ is a trinomial or pentanomial [GK03, ALH10a, ALH10b].

For completeness, we explain here the principle of modular reduction by a trinomial. The method for pentanomials uses the same idea, and it is explained in Appendix A. Let $f_{3}(x)=x^{n}+x^{k_{1}}+1$ such that $0<k_{1} \leq\left\lceil\frac{n}{2}\right\rceil$. Let $R_{0}=\sum_{i=0}^{n-1} r_{i} x^{i}, R_{k_{1}}=\sum_{i=n}^{2 n-k_{1}-1} r_{i} x^{i-n}$ and $S_{k_{1}}=\sum_{i=2 n-k_{1}}^{2 n-2} r_{i} x^{i-2 n+k_{1}}$, we have:

$$
R=R_{0}+\left(R_{k_{1}}+S_{k_{1}} x^{n-k_{1}}\right) x^{n} .
$$

We do a first step of reduction by $f_{3}$ by replacing $x^{n}$ by $f_{3}(x)-x^{n}$ in Equation (6). We obtain:

$$
R=R_{0}+\left(R_{k_{1}}+S_{k_{1}} x^{n-k_{1}}\right)+\left(R_{k_{1}} x^{k_{1}}+S_{k_{1}} x^{n}\right) \bmod f_{3} .
$$

We iterate a new step of reduction:

$$
R=R_{0}+R_{k_{1}}+S_{k_{1}} x^{n-k_{1}}+R_{k_{1}} x^{k_{1}}+S_{k_{1}}\left(f_{3}(x)-x^{n}\right) \bmod f_{3} .
$$

In Equation (7), the degree of $R$ is $\max \left(n-1,2\left(k_{1}-1\right)\right)$. So, $R$ is reduced modulo $f_{3}$ only if $2\left(k_{1}-1\right)<n$. In two steps of reduction, we have then a method to compute the modular reduction for all trinomial such that $2\left(k_{1}-1\right)<n$.

To optimize the computation of $(7)$, we factorize by $\left(f_{3}(x)-x^{n}\right)$. In this way, we can rewrite $R$ as:

$$
R=R_{0}+S_{k_{1}} x^{n-k_{1}}+\left(R_{k_{1}}+S_{k_{1}}\right)\left(f_{3}(x)-x^{n}\right) \bmod f_{3} .
$$

There are mainly two methods [BG13] to compute (8). The first is the shift-and-add strategy: $\left(R_{k_{1}}+S_{k_{1}}\right)\left(f_{3}(x)-x^{n}\right)$ is computed as $Q+Q x^{k_{1}}$ with $Q=R_{k_{1}}+S_{k_{1}}$. The second is the mul-and-add strategy: the multiplication by $\left(f_{3}(x)-x^{n}\right)$ is computed with the PCLMULQDQ instruction. In this case, it is recommended to choose $k_{1}$ strictly less than 64 . In this way, $\left(f_{3}(x)-x^{n}\right)$ can be directly used as one of the operand of PCLMULQDQ instruction. We choose the first method because it requires a small number of low cost instructions.

These methods can be optimized for specific values of $k_{1}$ and $n$. Firstly, the case $k_{1}=1$ permits to avoid computations because $S_{k_{1}}=0$. Secondly, a classical trick is the use of the PSLLDQ and PSRLDQ instructions which permit to shift 128-bit registers by a multiple of 8 bits. The PALIGNR instruction can also be used. As it is explained in Section 1.7, it permits to concatenate two registers 128 bits, then to extract 16 contiguous bytes. We list here cases where these instructions could be used:

- For the extraction of $R_{k_{1}}$ from $R$ when $n$ is a multiple of 8. However, it does not exist irreducible trinomials such that $n$ is a multiple of 8 [Swa62].

- For the extraction of $S_{k_{1}}$ from $R$ when $2 n-k_{1}$ is a multiple of 8 .

- To obtain $S_{k_{1}} x^{n-k_{1}}$ from $S_{k_{1}}$ when $n-k_{1}$ is a multiple of 8 .

- For the multiplication by $x^{k_{1}}$ when $k_{1}$ is a multiple of 8 . 
For the shift-and-add strategy, we can also compute $\left(R_{k_{1}}+S_{k_{1}}\right)\left(f_{3}(x)-x^{n}\right)$ as $\frac{Q^{\prime}}{x^{k_{1}}}+Q^{\prime}$ with $Q^{\prime}=R_{k_{1}} x^{k_{1}}+S_{k_{1}} x^{k_{1}}$. In this case, the 128-bit shifts improve the implementation in the following cases:

- For the extraction of $R_{k_{1}} x^{k_{1}}$ from $R$ when $n-k_{1}$ is a multiple of 8 .

- For the extraction of $S_{k_{1}} x^{k_{1}}$ from $R$ when $2 n-2 k_{1}$ is a multiple of 8 .

- To obtain $S_{k_{1}} x^{n-k_{1}}$ from $S_{k_{1}} x^{k_{1}}$ when $n-2 k_{1}$ is a multiple of 8 .

- For the division by $x^{k_{1}}$ when $k_{1}$ is a multiple of 8 .

We have implemented the shift-and-add method for trinomials with different SIMD instruction sets. ssse3 is used to improve the implementation with the PALIGNR instruction. We have also implemented the shift-and-add method for pentanomials, but it is vectorized only for $n \in\{184,312,448,544\}$, because they are the parameters of Gui and DualModeMS256. For the implementation of HFE-based schemes, we estimate that the best strategy is to choose $n$ such that it exists an irreducible trinomial of degree $n$.

The performance of the modular reduction depends on the context. In Table 7, we reduce products from a small buffer, then we measure the cost of one modular reduction on average. We have removed the optimizations using PSLLDQ and PSRLDQ to have comparable measurements. In practice, they are used (for example, the ssse3 modular reduction for $n=375$ takes 12.2 cycles with these optimizations). The ssse 3 version is two times faster that without vector instructions because sse2 permits to perform two 64-bit instructions in one instruction. The avx2 implementation is slightly faster than ssse 3 version, probably because the avx2 is faster to load and store data.

Table 7: Number of cycles to compute the modular reduction of an element $\mathbb{F}_{2}[X]$ of degree $2 n-2$ by $f$, with MQsoft. We use a Skylake processor (LaptopS).

\begin{tabular}{|l|l|l|l|}
\hline \multirow{2}{*}{$\left(n, k_{1}\right)$} & \multicolumn{3}{|c|}{ Rem } \\
\cline { 2 - 4 } & Without SIMD & ssse3 & avx2 \\
\hline$(62,29)$ & $\mathbf{6 . 6}$ & $\mathbf{6 . 6}$ & $\times$ \\
\hline$(126,21)$ & 10.9 & $\mathbf{7 . 2}$ & $\times$ \\
\hline$(191,9)$ & 14.5 & $\mathbf{1 0 . 7}$ & 10.8 \\
\hline$(252,15)$ & 19.2 & 11.1 & $\mathbf{1 0 . 2}$ \\
\hline$(314,15)$ & 24.1 & $\mathbf{1 4 . 6}$ & $\times$ \\
\hline$(375,16)$ & 29.5 & $\mathbf{1 4 . 9}$ & $\times$ \\
\hline$(441,7)$ & 34.2 & $\mathbf{1 7 . 8}$ & $\times$ \\
\hline$(511,10)$ & 37.1 & 18.6 & $\mathbf{1 6 . 9}$ \\
\hline$(574,13)$ & 40.3 & $\mathbf{2 4 . 5}$ & $\times$ \\
\hline
\end{tabular}

In Table 8, the modular reduction is also measured when it is used with multiplication in $\mathbb{F}_{2}[X]$. The performance of multiplication in $\mathbb{F}_{2^{n}}$ depends on the context. For this reason, we measure it in two ways:

- Left value: we measure the cost of one field multiplication on average during the computation of the naive exponentiation function $\left(x^{i}\right.$ is computed as $\left.x^{i-1} x\right)$. Each result depends on the previous result, and the data are already loaded.

- Right value: we measure the cost of one field multiplication on average to compute the multiplication of elements of two buffers. The data are independent but each multiplication requires to load input and to store output. 
Table 8: Number of cycles to compute the multiplication in $\mathbb{F}_{2^{n}}$ in function of the modular reduction, with MQsoft. We use a Skylake processor (LaptopS).

\begin{tabular}{|l|l|l|l|l|}
\hline \multirow{2}{*}{$\left(n, k_{1}\right)$} & \multicolumn{4}{|c|}{ Rem } \\
\cline { 2 - 5 } & \multicolumn{2}{|c}{ ssse3 } & \multicolumn{2}{c|}{ avx2 } \\
\cline { 2 - 5 } & exp. & buffer & exp. & buffer \\
\hline$(62,29)$ & $\mathbf{1 7 . 3}$ & $\mathbf{8 . 5}$ & $\times$ & $\times$ \\
\hline$(126,21)$ & $\mathbf{2 6 . 6}$ & $\mathbf{1 4 . 8}$ & $\times$ & $\times$ \\
\hline$(191,9)$ & $\mathbf{3 2 . 8}$ & $\mathbf{2 5 . 9}$ & 44.7 & 27.2 \\
\hline$(252,15)$ & $\mathbf{4 0 . 2}$ & $\mathbf{3 5 . 6}$ & 53.7 & 36.9 \\
\hline$(314,15)$ & $\mathbf{5 5 . 0}$ & $\mathbf{5 1 . 5}$ & $\times$ & $\times$ \\
\hline$(375,16)$ & $\mathbf{6 5 . 9}$ & $\mathbf{6 5 . 8}$ & $\times$ & $\times$ \\
\hline$(441,7)$ & $\mathbf{8 0 . 2}$ & $\mathbf{8 2 . 8}$ & $\times$ & $\times$ \\
\hline$(511,10)$ & $\mathbf{9 0 . 7}$ & $\mathbf{9 1 . 5}$ & 103.7 & 95.3 \\
\hline$(574,13)$ & $\mathbf{1 1 4 . 9}$ & $\mathbf{1 2 0 . 3}$ & $\times$ & $\times$ \\
\hline
\end{tabular}

Table 9: Number of cycles to compute the squaring in $\mathbb{F}_{2^{n}}$ in function of the enabled instructions, with MQsoft. We use a Skylake processor (LaptopS).

\begin{tabular}{|l|l|l|l|l|l|l|l|l|}
\hline \multirow{2}{*}{$\left(n, k_{1}\right)$} & \multicolumn{2}{|c|}{ PSHUFB } & \multicolumn{2}{c|}{ VPSHUFB } & \multicolumn{2}{c|}{ PCLMULQDQ } & \multicolumn{2}{c|}{ PCLMULQDQ, avx2 } \\
\cline { 2 - 9 } & multi-sqr & buffer & multi-sqr & buffer & multi-sqr & buffer & multi-sqr & buffer \\
\hline$(62,29)$ & $\mathbf{1 4 . 9}$ & 9.2 & $\times$ & $\times$ & 17.3 & $\mathbf{7 . 6}$ & $\times$ & $\times$ \\
\hline$(126,21)$ & $\mathbf{1 8 . 6}$ & 11.8 & $\times$ & $\times$ & 21.5 & $\mathbf{1 0 . 0}$ & $\times$ & $\times$ \\
\hline$(191,9)$ & 24.0 & 18.4 & $\times$ & $\times$ & $\mathbf{2 3 . 3}$ & $\mathbf{1 4 . 1}$ & 35.0 & 15.7 \\
\hline$(252,15)$ & 25.2 & 20.0 & 30.8 & 16.8 & $\mathbf{2 3 . 9}$ & $\mathbf{1 4 . 7}$ & 37.4 & 16.3 \\
\hline$(314,15)$ & 29.1 & 26.3 & $\times$ & $\times$ & $\mathbf{2 5 . 2}$ & $\mathbf{2 2 . 0}$ & $\times$ & $\times$ \\
\hline$(375,16)$ & 28.9 & 27.0 & $\times$ & $\times$ & $\mathbf{2 5 . 8}$ & $\mathbf{2 1 . 3}$ & $\times$ & $\times$ \\
\hline$(441,7)$ & 34.8 & 34.8 & $\times$ & $\times$ & $\mathbf{2 7 . 4}$ & $\mathbf{2 5 . 1}$ & $\times$ & $\times$ \\
\hline$(511,10)$ & 38.4 & 36.4 & 35.7 & 28.7 & $\mathbf{2 6 . 7}$ & $\mathbf{2 4 . 2}$ & 39.8 & 28.0 \\
\hline$(574,13)$ & 45.5 & 43.5 & $\times$ & $\times$ & $\mathbf{2 9 . 7}$ & $\mathbf{3 1 . 2}$ & $\times$ & $\times$ \\
\hline
\end{tabular}

We remark that for $n$ less than 375 , the multiplication on independent data is faster. It is probably caused by the latency of the PCLMULQDQ instruction. The field multiplication with modular reduction using sse2 is the fastest, because the PCLMULQDQ instruction requires to use 128-bit registers. When sse2 is used with avx2, the implementation pays a penalty. However, this problem will be solved with the VPCLMULQDQ instruction on the future Ice Lake processors [Int18].

In Table 9 , the modular reduction is measured when it is used with squaring in $\mathbb{F}_{2}[X]$. As for the multiplication in $\mathbb{F}_{2^{n}}$, the performance of squaring depends on the context. For this reason, we measure it in two ways:

- Left value: we measure the cost of one field squaring on average during the raising of an element of $\mathbb{F}_{2^{n}}$ at the power $2^{i}\left(x^{2^{i}}\right.$ is computed as $\left.\left(x^{2^{i-1}}\right)^{2}\right)$. Each result depends on the previous result, and the data are already loaded.

- Right value: we measure the cost of one field squaring on average to compute the squaring of elements of one buffer. The data are independent but each squaring requires to load input and to store output.

Table 9 shows the performance of squaring in $\mathbb{F}_{2^{n}}$. The squaring using PCLMULQDQ is the most efficient. For the same reasons that for the multiplication in $\mathbb{F}_{2^{n}}$, the best modular 
squaring is the one using only sse2 modular reduction. This is the default setting in MQsoft.

All measured functions in this Section are available is our library. They are implemented in constant-time. The modular reduction by pentanomials is also available to make the library more complete, but is not yet vectorized (excepted for $n \in\{184,312,448,544\}$ ).

\subsection{Multi-squaring in $\mathbb{F}_{2^{n}}$}

The multi-squaring $\left[\mathrm{TFA}^{+} 11\right]$ is an operation computing successively several squarings. This operation is important to compute the inverse in $\mathbb{F}_{2^{n}}^{\times}$(in Section 2.5). Algorithm 5 requires to compute $B^{2^{i}}$, for $B \in \mathbb{F}_{2^{n}}$ and various values of $i$. For small $i$, the best way is to raise $B$ at the power two $i$ times (as in Section 2.3). For larger $i$, the best method is to use precomputed multi-squaring tables. Let $B=\sum_{k=0}^{n-1} \alpha_{k} x^{k} \in \mathbb{F}_{2^{n}}$ for $\mathbb{F}_{2^{n}}=\mathbb{F}_{2} /(f(x))$ (Section 1.6), then $B^{2^{i}}=\left(\sum_{k=0}^{n-1} \alpha_{k} x^{k 2^{i}}\right) \bmod f$. The idea of multi-squaring tables is to store $x^{k 2^{i}} \bmod f$ for $k \in \llbracket 0, n-1 \rrbracket$. Then, multi-squaring is equivalent to the dot product of the vectors $\left(\alpha_{0}, \ldots, \alpha_{n-1}\right)$ and $\left(1, x^{2^{i}} \bmod f, \ldots, x^{(n-1) 2^{i}} \bmod f\right)$. The table requires to store $n-1$ elements in $\mathbb{F}_{2^{n}}$ ( 1 is not formally stored), and the multi-squaring requires $n-1$ multiplications between elements of $\mathbb{F}_{2}$ and $\mathbb{F}_{2^{n}}$ and $n-1$ additions in $\mathbb{F}_{2^{n}}$. In a variable-time implementation, the multiplication by $\alpha_{k}$ can be done by a conditional statement. In a constant-time implementation, the value of $\alpha_{k}$ is duplicated in the mask variable, in the way to replace the multiplication by a bitwise AND with this mask. This process is explained in Section 4.2.

In variable-time implementation, the performance can be improved with larger tables [Mai15]. Rather that to compute $\alpha_{k} x^{k 2^{i}} \bmod f$ coefficient by coefficient, the coefficients can be grouped by block of $B$, and the $2^{B}$ possibility of $\sum_{k=0}^{B-1} \alpha_{j B+k} x^{(j B+k) 2^{i}}$ can be precomputed for $j \in \llbracket 0,\left\lceil\frac{n}{B}\right\rceil-1 \rrbracket$. This method cannot be used in a constant-time implementation because of the timing attack on the memory latency. It permits to attack the index of the precomputed table. In our implementation, we use a constant-time implementation of multi-squaring.

\subsection{Modular Inverse in $\mathbb{F}_{2^{n}}^{\times}$}

The computation of the inverse in $\mathbb{F}_{2^{n}}^{\times}$is often required for the arithmetic in $\mathbb{F}_{2^{n}}[X]$. In our case, it is required to compute the GCD (Section 3.6). To compute the modular inverse of $A \in \mathbb{F}_{2^{n}}^{\times}$, there are mainly two methods. The first is to use extended Euclidean algorithm (EEA) [vzGG13, Algorithm 3.6]. This method is not constant-time. The second is to compute $A^{-1}=A^{2^{n}-2}$ by Fermat's little theorem. The exponentiation can be done with the square and multiply method [vzGG13, Algorithm 4.8], costing $n-1$ squarings and $n-2$ multiplications in $\mathbb{F}_{2^{n}}$. The Itoh-Tsujii Multiplicative Inversion Algorithm (ITMIA) [IT88] permits to modify the way to compute the power with an addition chain. It requires $n-1$ squarings and only $O\left(\log _{2}(n)\right)$ multiplications in $\mathbb{F}_{2^{n}}$. The number of multiplications depends on the length of the chosen addition chains. ITMIA is described in Algorithm 5 for a specific addition chain which consists to read the bits of $n-1$ from MSB to LSB. At the end of the each iteration, the variable inv is always $A^{2^{\text {val }}-1}$. 




ITMIA requires to compute val successive squarings. The multi-squaring can be computed more quickly with precomputed tables (cf. Section 2.4).

Algorithm 5 is useful because it proposes automatically an addition chain for all values of $n$, but it is not always optimal. To choose the best addition chain is not easy, because it depends on the performance of multiplication, squaring and multi-squaring. Moreover, there is a large set of possible addition chains. This problem is studied in [Mai15], which proposes a software generating an efficient $\mathrm{C}++$ inversion code. This software searches the addition chain which maximizes the performance of the generated code. However, the generator does not proposes implementation of multi-squaring tables in constant-time. For the moment, MQsoft uses Algorithm 5, but we propose in Appendix B examples of addition chains chosen to minimize the number of field multiplications. We have improved Algorithm 5 with multi-squaring tables to compute the variable tmp when $i$ is zero or one. Because multi-squaring tables are huge, we use it only for the parameters of GeMSS, Gui, DualModeMS, and for the values of $n$ used to evaluate the performance of MQsoft. The corresponding file in MQsoft requires 1.7 MB for 44 tables.

\subsection{Performance of the Arithmetic in $\mathbb{F}_{2^{n}}$}

Table 10 compares the performance of arithmetic operations in our library with respect to several open source libraries (listed in Section 1.7). We choose the irreducible trinomial $f(x)=x^{n}+x^{k_{1}}+1$ with $k_{1} \in \llbracket 2,32 \rrbracket$ to create the field $\mathbb{F}_{2^{n}}$. All operations use modular reduction. We have measured the performance of FLINT [Har10, version 2.5.3] (it is a C library), but the times are not relevant in our context. It turns that for $n=252$, NTL is 100 to 200 times faster than FLINT. The main reason is that FLINT does not have special implementation for binary fields. We have used the type fq_nmod_t which store each element of $\mathbb{F}_{2^{n}}$ as a polynomial in $\mathbb{F}_{2}[X]$ where each coefficient is stored on one word. Magma is also taken into account. The results are not significant because Magma is slowed down by its user interface. We remark that the squarings and multiplications of NTL are faster for $n=126$ than for $n=62$. It can be probably explained by the fact that NTL does not use trinomial for $n=62$. Our implementation is 3.5 to 4.5 times faster than NTL for multiplication and 5 to 6 times faster for squaring. We think that NTL is slowed down by its $\mathrm{C}++$ interface. For the inversion, the measurements are not comparable because NTL is not in constant-time. However, we have a speed-up of two on average.

We compare now MQsoft to the constant-time arithmetic of [BG13], when trinomials are used to build $\mathbb{F}_{2^{n}}$. In $\mathbb{F}_{2^{233}}$, they compute the squaring in 18 cycles and the multiplication in 38 cycles. We have approximately the same performance for $n=252$ : MQsoft is slower 
Table 10: Number of cycles by operation in $\mathbb{F}_{2^{n}}$. We use a Skylake processor (LaptopS).

\begin{tabular}{|c|c|c|c|c|c|}
\hline \multirow[t]{2}{*}{$\left(n, k_{1}\right)$} & \multirow[t]{2}{*}{ Operation } & \multirow[t]{2}{*}{ Magma } & \multirow[t]{2}{*}{ NTL } & \multicolumn{2}{|c|}{ MQsoft (PCLMULQDQ + avx2) } \\
\hline & & & & dependencies & buffer \\
\hline \multirow[t]{3}{*}{$(62,29)$} & Squaring & 416 & 220 & 14.9 & 7.6 \\
\hline & Mul & 444 & 231 & 17.3 & 8.5 \\
\hline & Inverse & 13,183 & 1,868 & $x$ & 1,154 \\
\hline \multirow[t]{3}{*}{$(126,21)$} & Squaring & 440 & 105 & 18.6 & 10.0 \\
\hline & Mul & 494 & 124 & 26.6 & 14.8 \\
\hline & Inverse & 27,353 & 3,457 & $x$ & 1,731 \\
\hline \multirow[t]{3}{*}{$(191,9)$} & Squaring & 437 & 119 & 23.3 & 14.1 \\
\hline & Mul & 529 & 144 & 32.8 & 25.9 \\
\hline & Inverse & 40,200 & 4,918 & $x$ & 2,706 \\
\hline \multirow[t]{3}{*}{$(252,15)$} & Squaring & 455 & 128 & 23.9 & 14.7 \\
\hline & Mul & 558 & 169 & 40.2 & 35.6 \\
\hline & Inverse & 51,720 & 7,809 & $x$ & 3,647 \\
\hline \multirow[t]{3}{*}{$(314,15)$} & Squaring & 480 & 139 & 25.2 & 22.0 \\
\hline & Mul & 629 & 211 & 55.0 & 51.5 \\
\hline & Inverse & 66,220 & 9,515 & $\times$ & 5,096 \\
\hline \multirow[t]{3}{*}{$(375,16)$} & Squaring & 490 & 150 & 25.8 & 21.3 \\
\hline & Mul & 653 & 238 & 65.9 & 65.8 \\
\hline & Inverse & 76,704 & 11,813 & $x$ & 6,364 \\
\hline \multirow[t]{3}{*}{$(441,7)$} & Squaring & 500 & 163 & 27.4 & 25.1 \\
\hline & Mul & 714 & 286 & 80.2 & 82.8 \\
\hline & Inverse & 94,846 & 14,974 & $\times$ & 7,755 \\
\hline \multirow[t]{3}{*}{$(511,10)$} & Squaring & 510 & 174 & 26.7 & 24.2 \\
\hline & Mul & 761 & 320 & 90.7 & 91.5 \\
\hline & Inverse & 115,681 & 18,601 & $x$ & 8,825 \\
\hline \multirow[t]{3}{*}{$(574,13)$} & Squaring & 521 & 201 & 29.7 & 31.2 \\
\hline & Mul & 922 & 579 & 114.9 & 120.3 \\
\hline & Inverse & 129,266 & 23,185 & $x$ & 11,329 \\
\hline
\end{tabular}

with dependencies but faster with buffers. In $\mathbb{F}_{2^{409}}$, they compute the squaring in 28 cycles and the multiplication in 97 cycles. MQsoft is slightly faster (we compare to the measurements in $\mathbb{F}_{2^{441}}$ ). For the inversion, [BG13] is approximately two times slower. Our library takes advantage of using of multi-squaring tables. We replace $n-1$ squarings by approximately $\frac{1}{4} n$ squarings and two multi-squarings.

\section{Efficient Implementation of Root Finding in $\mathbb{F}_{2^{n}}[X]$}

The most expensive part of the signature generation is to find the roots of a HFE polynomial $F \in \mathbb{F}_{2^{n}}[X]$ as defined in Equation (2). $F$ is a $D$ degree monic polynomial which is sparse because it has approximately $\frac{\log _{2}(D)^{2}}{2}$ non-zero coefficients. We have chosen to implement Berlekamp's algorithm [vzGG13, Algorithm 14.15] which finds the roots with an asymptotic complexity of $O\left(n D^{2}+(n+\log (s)) s^{2} \log (s)\right)$ operations in $\mathbb{F}_{2^{n}}$, where $s$ is the number of roots of $F$ [vzGG13, Theorem 14.11 adapted for $r=s$ and $d=1$ ]. For HFE polynomials, the factor $O\left(n D^{2}\right)$ can be easily improved in $O\left(n D \log _{2}(D)^{2}+D^{2}\right)$ operations in $\mathbb{F}_{2^{n}}$, by using the sparse structure of $F$ (Section 3.5). Moreover, the HFE polynomial does not have many roots, so we can assume that $s$ is negligible, yielding a final complexity of $O\left(n D \log _{2}(D)^{2}+D^{2}\right)$ operations in $\mathbb{F}_{2^{n}}$. 
For the general polynomials, the author of [Pet14] proposes in 2014 the successive resultant algorithm (SRA). It requires $O\left(n^{3} D^{2}+n^{4}\right)$ operations in $\mathbb{F}_{2}$ to find roots, or $\widetilde{O}\left(n^{2} D+n^{3}\right)$ with the fast arithmetic. The step in $O\left(n^{4}\right)$ (or $\left.\widetilde{O}\left(n^{3}\right)\right)$ can be precomputed for a fixed finite field. In comparison to Berlekamp, SRA is interesting only when the polynomial has many roots. In [GvdHL15] and [DPP16], the root finding is improved for split and separable polynomials, and when the cardinality of multiplicative group is smooth. In our case, this method is not interesting because the HFE polynomials does not have many roots.

Improving root finding for sparse polynomials is a hard problem. In [BCR13], the authors propose the first sub-linear (in $q$ ) algorithm which detects the existence of roots for $t$ monomials in $\mathbb{F}_{q}[X]$. The complexity is of $4^{t+o(1)} q^{\frac{t-2}{t-1}+o(1)}$ bit operations. This method is not interesting for a HFE polynomial because it is not enough sparse and also because in practice $n$ is greater that the level of security. The algorithm costs approximately $4^{o(1)} 2^{n+o(n)} D^{\log _{2}(D)}$ bit operations in our case.

In this section, we will remind the Berlekamp's algorithm. For each operation required, we study the different possible methods and compare their practical performance to choose the best. We specify how these methods can be tuned for a HFE polynomial.

\subsection{Description of Berlekamp's Algorithm in $\mathbb{F}_{2^{n}}[X]$}

Algorithm 6 describes Berlekamp's algorithm [vzGG13, Algorithm 14.15]. The main idea is to remark that all elements of $\mathbb{F}_{2^{n}}$ vanish on $X^{2^{n}}-X$. We can then compute $G$ the GCD on $F$ with $X^{2^{n}}-X$. $G$ has the same roots than $F$ but with a minimal degree (which is the number of roots). In general, the degree of $G$ is small. The strategy is then to apply the so-called equal-degree factorization to find all roots. This is turned to be cheap. Indeed, let $s$ be the degree of $G$, the equal-degree factorization costs $(n+\log (s)) s^{2} \log (s)$ operations in $\mathbb{F}_{2^{n}}$ [vzGG13, Theorem 14.11 adapted for $r=s$ and $\left.d=1\right]$. Because the degree of $X^{2^{n}}-X$ is big, we reduce $X^{2^{n}}-X$ by $F$ by using the repeated squaring algorithm in $\mathbb{F}_{2^{n}}[X]$, before computing the GCD.

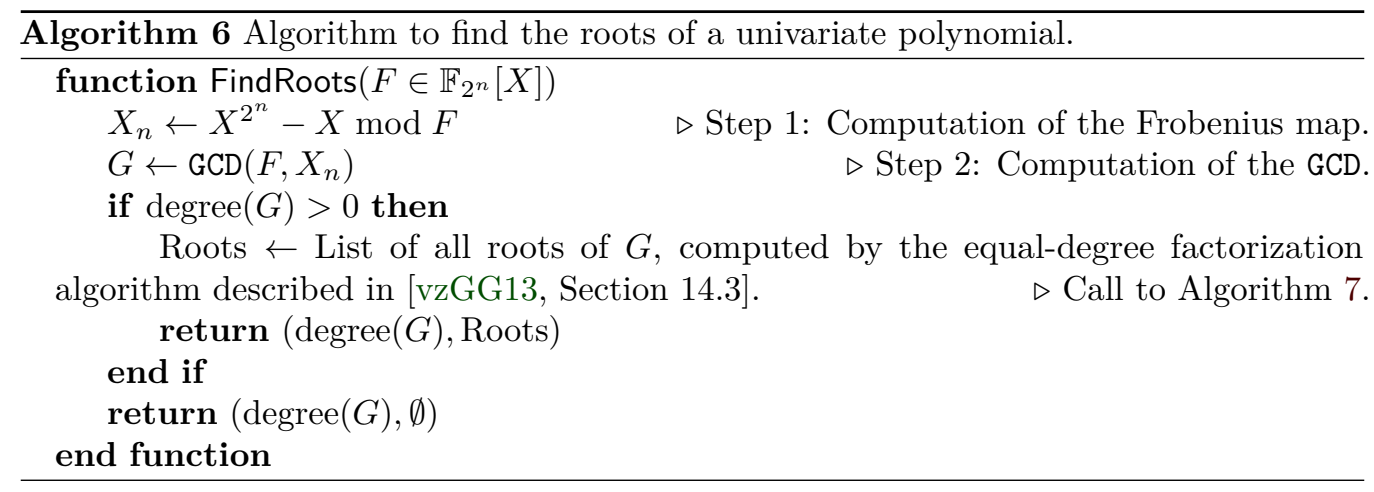

Thereafter, we will study the choice of the algorithms only for the steps one and two. The computation of equal-degree factorization is negligible since $G$ has a small degree. For the set of completeness, the equal-degree factorization algorithm is summarized in Algorithm 7. We will compare the classical and fast algorithms and we optimize them for a HFE polynomial which is sparse, i.e. which has $O\left(\log _{2}\left(D^{2}\right)\right)$ coefficients in comparison to a dense polynomial that has $D+1$ coefficients. 


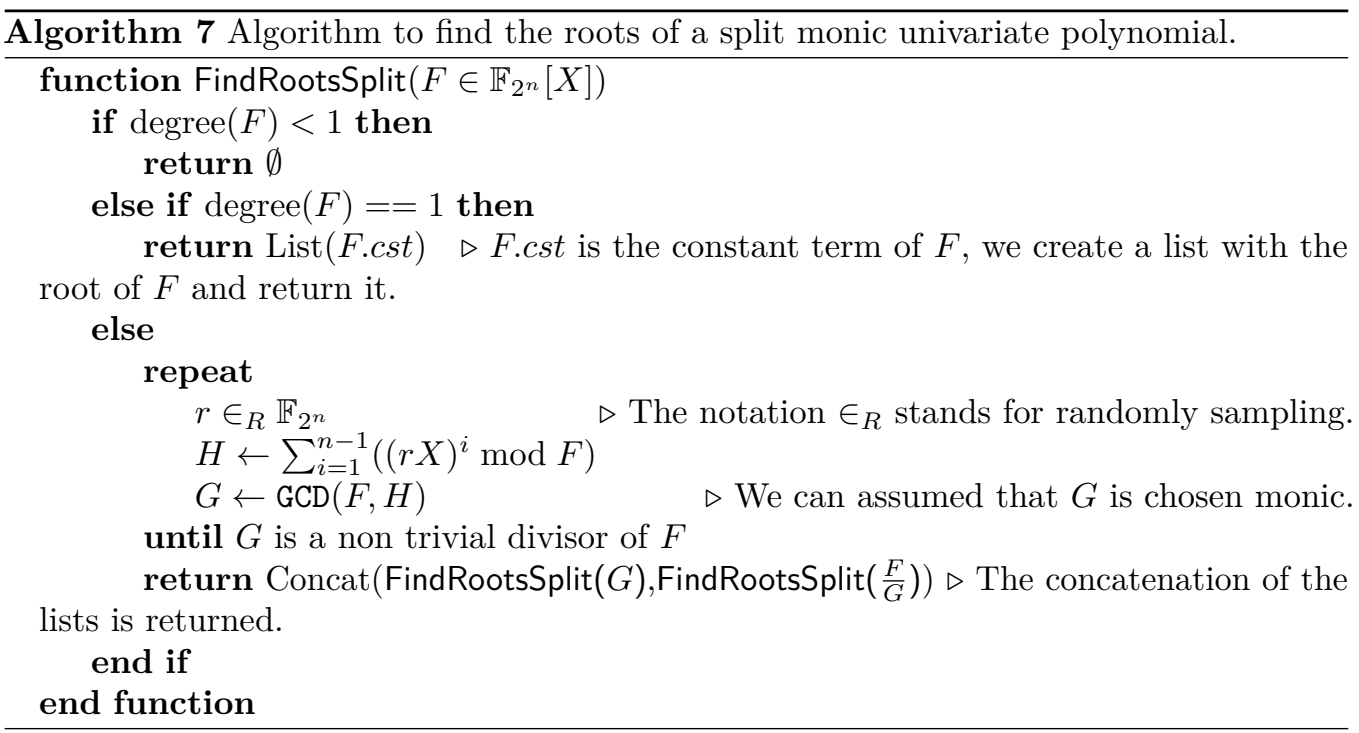

\subsection{Polynomial Squaring in $\mathbb{F}_{2^{n}}[X]$}

Step 1 of Algorithm 6 requires to compute repeated squarings in $\mathbb{F}_{2^{n}}[X]$. In binary fields, squaring of $B=\sum_{i=0}^{D-1} \alpha_{i} X^{i} \in \mathbb{F}_{2^{n}}[X]$ is linear. Similarly to Section 2.1 , it holds that $B^{2}=\sum_{i=0}^{D-1} \alpha_{i}^{2} X^{2 i}$. This operation requires $D$ squaring in $\mathbb{F}_{2^{n}}$.

\subsection{Euclidean Division in $\mathbb{F}_{2^{n}}[X]$}

Let $P \in \mathbb{F}_{2^{n}}[X]$ be a univariate polynomial. The notation $\operatorname{coef}_{i}(P)$ will denote the coefficient of the term of degree $i$ in $P$. Step 1 of Algorithm 6 requires to compute the modular reduction of a polynomial $P$ of degree at most $2 D-2$ by a monic polynomial $F$. The classical algorithm [vzGG13, Algorithm 2.5] uses $O\left(D^{2}\right)$ multiplications in $\mathbb{F}_{2^{n}}$.

Algorithm 8 computes the modular reduction specialized for HFE polynomials. Let $K$ be the number of terms of $F_{\mathrm{HFE}}$ (Section 1.6). For a fixed $i$, each term of $\left(F_{\mathrm{HFE}}-X^{D}\right) X^{i-D}$ is multiplied by $-\operatorname{coef}_{i-D}(Q)$. Then, the result is added to $R$, requiring $K-1$ multiplications and additions in $\mathbb{F}_{2^{n}}$. Since $K-1=O\left(\log _{2}(D)^{2}\right)$ coefficients, the Euclidean division requires $(D-1)(K-1)=O\left(D \log _{2}(D)^{2}\right)$ multiplications in $\mathbb{F}_{2^{n}}$. This method is in constant-time because we compute the multiplication by $\operatorname{coef}_{i-D}(Q)$ even when it is null.

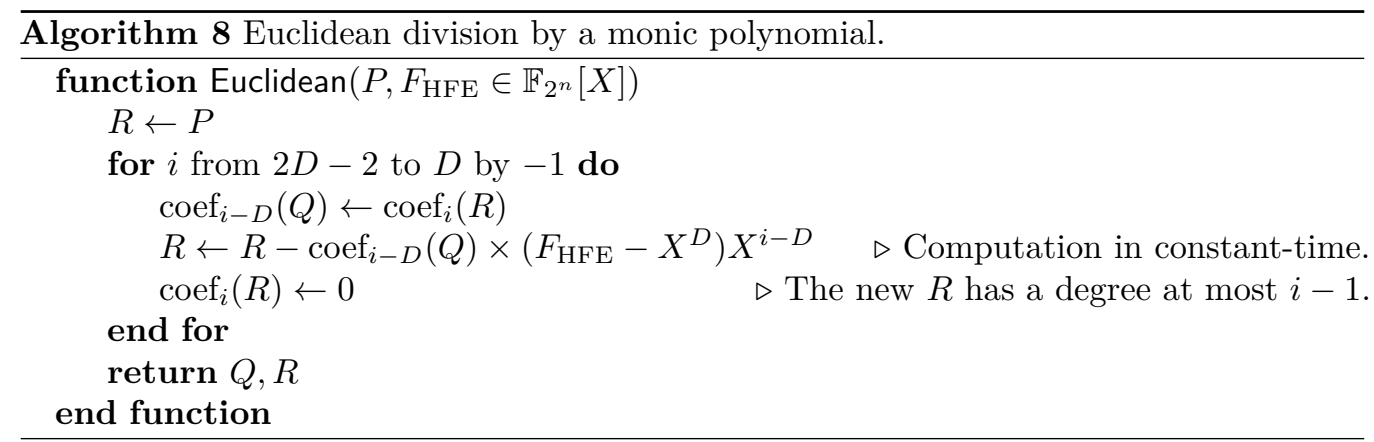




\subsection{Improving Euclidean Division for Special HFE Polynomials}

The previous method does not exploit that during Step 1 of Algorithm 6, the dividend is a square. Terms of odd degree are null. We show here that the complexity can be divided by two, in function of the term of higher odd degree of the divisor. For this, we introduce a new notation. Let $P \in \mathbb{F}_{2^{n}}[X]$, we denote by $\mathcal{D}(P)$ the largest odd integer $i$ such that $\operatorname{coef}_{i}(P) \neq 0$. If it does not exist, we set $\mathcal{D}(P)=-\infty$. The following lemma permits to demonstrate the main result (Theorem 1) of this part.

Lemma 1. Let $A \in \mathbb{F}_{2^{n}}[X]$ be a polynomial of degree at most $2 D-2$ such that $\mathcal{D}(A)=-\infty$, $H \in \mathbb{F}_{2^{n}}[X]$ be of degree $D, Q, R \in \mathbb{F}_{2^{n}}[X]$ be respectively the quotient and remainder of the Euclidean division of $A$ by $H$ and $d=\mathcal{D}(H)$. If $D$ is even then $\mathcal{D}(Q) \leq d-2$.

Proof. Let $H=\sum_{j=0}^{D} h_{j} X^{j}$ and $Q=\sum_{j=0}^{D-2} q_{j} X^{j}$. By definition of $\mathcal{D}, \mathcal{D}(Q) \leq d-2$ is equivalent to $q_{i}=0$ for all odd $i$ such that $i>d-2$. By definition of $Q, q_{i}=0$ for $i<0$ and $i>D-2$, so we show the lemma for the values of $q_{i}$ such that $D-2 \geq i>\max (-1, d-2)$. For this, we use a proof by induction on an odd $j$ such that $D-1 \geq j>\max (-1, d-2)$. The base case is trivial since $q_{j}=0$ for $j>D-2$. Assume that $q_{k}=0$ for all odd $k$ such that $D-2 \geq k>j>\max (-1, d-2)$. To show that $q_{j}=0$, firstly we show these two properties:

(1) $\operatorname{coef}_{D+j}(H Q)=0$.

(2) $\operatorname{coef}_{D+j}(H Q)=q_{j} h_{D}$.

Proof of these two properties:

(1) By definition, $A=H Q+R$ and so $A-R=H Q$. Because $\mathcal{D}(A)=-\infty$ by hypothesis, $\mathcal{D}(A-R)=\mathcal{D}(R) \leq D-1<D+j$ and $D+j$ is odd so $\operatorname{coef}_{D+j}(A-R)=0$.

(2) $H Q=\sum_{r=0}^{2 D-2} \sum_{\ell=0}^{r} q_{\ell} h_{r-\ell} X^{r}$, so $\operatorname{coef}_{D+j}(H Q)=\sum_{\ell=0}^{D+j} q_{\ell} h_{D+j-\ell}$. But $q_{\ell}=0$ for $\ell>D-2$ and $h_{D+j-\ell}=0$ for $D+j-\ell>D$, so $\operatorname{coef}_{D+j}(H Q)=\sum_{\ell=j}^{D-2} q_{j} h_{D+j-\ell}$. When $\ell>j$ is odd, $q_{\ell}=0$ by induction hypothesis. When $\ell$ is even, $h_{D+j-\ell}=0$ because $D+j-\ell$ is odd and $\mathcal{D}(H)=d<D+j-\ell$. So $\sum_{\ell=j}^{D-2} q_{\ell} h_{D+j-\ell}=q_{j} h_{D}$.

These two properties implies $\operatorname{coef}_{D+j}(H Q)=q_{j} h_{D}=0$. Because $h_{D} \neq 0$, this implies that $q_{j}=0$.

We can now demonstrate Theorem 1.

Theorem 1. Let $H$ be a HFE polynomial of degree $D$ in $\mathbb{F}_{2^{n}}[X]$ where the $k$-th terms of highest odd degree have been removed $\left(k \in \llbracket 0,\left\lceil\log _{2}(D)\right\rceil-1 \rrbracket\right)$, and let $A \in \mathbb{F}_{2^{n}}[X]$ be a square of degree at most $2 D-2$. If $D$ is even, then the computation of the classical Euclidean division (Algorithm 8) of $A$ by $H$ can be accelerated by a factor $(D-1) /\left(\frac{D}{2}+\right.$ $\left.\left\lfloor 2^{\left\lceil\log _{2}(D)\right\rceil-k-2}\right\rfloor\right)$.

Proof. During Algorithm $8, A$ is a square so $\mathcal{D}(A)=-\infty$, and so Lemma 1 can be applied it. Let $d=\mathcal{D}(H)$, the iterations where $i$ is odd and strictly greater than $D+d-2$ can be removed because $\operatorname{coef}_{i-D}(Q)=0$. So, the number of iterations when $i$ is odd is $\max \left(\frac{(D+d-2)-(D-1)}{2}, 0\right)=\max \left(\frac{d-1}{2}, 0\right)$, whereas the number of iterations when $i$ is even is $\frac{D}{2}$. So, Algorithm 8 can be used with $\max \left(\frac{D+d-1}{2}, \frac{D}{2}\right)$ iterations. Next, $H$ is a HFE polynomial so $d=1$ or $d=2^{j}+1$ for $j>0$. By removing the $2^{j}+1$ degree terms for $j$ from $\left\lceil\log _{2}(D)\right\rceil-1$ to $\left\lceil\log _{2}(D)\right\rceil-k$ by $-1, d$ equals 1 or $2^{\left\lceil\log _{2}(D)\right\rceil-k-1}+1$. It implies that the number of iterations can be written as $\frac{D}{2}+\left\lfloor 2^{\left\lceil\log _{2}(D)\right\rceil-k-2}\right\rfloor$. Algorithm 8 requires $D-1$ iterations, so the proposed modification accelerates it of a factor $(D-1) /\left(\frac{D}{2}+\right.$ $\left.\left\lfloor 2^{\left\lceil\log _{2}(D)\right\rceil-k-2}\right\rfloor\right)$. This factor is at most 2 . 
Let $K$ be the number of terms of the HFE polynomial (without removed terms), and $k$ be the number of removed terms. For $k=0$, the modular reduction costs $(D-1)(K-1)$ multiplications in $\mathbb{F}_{2^{n}}$ (Section 3.3), whereas by removing terms (with even $D$ ), the cost is $\max \left(\frac{D+d-1}{2}, \frac{D}{2}\right)(K-1-k)$ multiplications. The main gain comes from the fact to decrease the number of round loops during Algorithm 8. However, there is also a slight speed-up generated by the fact that terms are removed.

When $F$ has exactly zero term of odd degree, we obtain that during the computation of $X^{2^{n}} \bmod F$, none of the odd degree terms appear because $R=A-F Q$ and $A, F$ and $Q$ do not have odd degree terms. This result allows to do computations only for even degree terms, dividing by 2 the cost of the squaring and of the modular reduction. But in practice, to remove all terms of odd degree of the HFE polynomial decreases the security. By applying to $F$ the change of variable $X^{2}=Y$, the degree of the result is only $\frac{D}{2}$. We will show in Table 11 that in this case, $D$ must be multiplied by two to obtain the original security.

Table 11 studies the impact of $d=\mathcal{D}(F)$ on the theoretical speed-up over the classical Euclidean division compared to the case $D=513$, and on the security. We have done an experimental test to analyse the degree of regularity [FJ03, BFS04, BFSY05, BFP13] in function of the number of removed terms. The degree of regularity is a tool to analyse the security of HFE-based scheme against Gröbner basis attacks. We measure it during the Gröbner basis attack on HFE for $n=m=30$. We observe in practice that removing a small number of odd degree terms appears not to affect the security. The security is decreased when the second to last term is removed. The results confirm that the security to attack $F$ of degree $D$ without odd degree terms is the same that attack a HFE polynomial of degree $\frac{D}{2}$ : the degree of regularity increases between $\frac{D}{2}=512$ and $\frac{D}{2}=513$. The case $d=1$ seems to have the same behavior, but in the general case, the regularity degree does not decrease necessarily (for $D=130$, the degree of regularity is 4 for $d=-\infty$ but 5 for $d=1$ ).

The column speed-up on $i$ corresponds to obtain speed-up by decreasing the number of iterations during Algorithm 8. The other column speed-up is the total speed-up, which uses the fact that remove terms decreases the number of multiplications for one iteration. To remove the higher terms generates the main part of the maximal speed-up. In practice, we propose to choose $d=63$. This implies to remove one term when $D=130$ and three terms when $D=514$.

\subsection{Frobenius Map in $\mathbb{F}_{2^{n}}[X]$}

The core of Algorithm 6 (Section 3.1) is to compute $X^{2^{n}} \bmod F$ during Step 1. As in Section 2.4, we compare the classical repeated squaring algorithm with the version using multi-squaring tables. The main differences with Section 2.4 is that the coefficients are not in $\mathbb{F}_{2}$ but in $\mathbb{F}_{2^{n}}$. So, the tables are too large to be precomputed. However, they can be computed more quickly by exploiting the HFE structure of $F$. Both presented methods are in constant-time.

Classical repeated squaring algorithm. We can compute $X^{2^{n}} \bmod F$ using repeated squaring algorithm [vzGG13, Algorithm 4.8]. This requires $n$ steps of modular squaring. More precisely, the number of steps is $n-\left\lfloor\log _{2}(D)\right\rfloor$ because the modular reduction is useless when the degree of $X^{2^{i}}, 1 \leq i \leq n$, is less than $D$. So, we compute $\left(X^{2^{\left\lfloor\log _{2}(D)\right\rfloor}}\right)^{2^{n-\left\lfloor\log _{2}(D)\right\rfloor}} \bmod F\left(\operatorname{or}\left(F-X^{D}\right)^{2^{n}-\left\lfloor\log _{2}(D)\right\rfloor} \bmod F\right.$ when $D$ is a power of two $)$. This remark permits to avoid useless computations in the constant-time implementations. This method requires $\left(n-\left\lfloor\log _{2}(D)\right\rfloor\right) D$ field squarings and $\left(n-\left\lfloor\log _{2}(D)\right\rfloor\right)$ call to Al- 
Table 11: Impact of $d$ on the performance and on $d_{\text {reg }}$ the degree of regularity.

\begin{tabular}{|c|c|c|c|c|c|c|}
\hline$D$ & $d$ & removed terms & $d_{\text {reg }}$ & nb. of iterations & speed-up on $i$ & speed-up \\
\hline 512 & 257 & none & 5 & 384 & $25 \%$ & $27 \%$ \\
\hline 513 & 513 & none & 6 & 512 & Ref. & Ref. \\
\hline \multirow[t]{10}{*}{514} & 257 & $X^{513}$ & \multirow[t]{8}{*}{6} & 385 & $25 \%$ & $25 \%$ \\
\hline & 129 & $X^{513}, X^{257}$ & & 321 & $37 \%$ & $39 \%$ \\
\hline & 65 & $X^{513}, X^{257}, X^{129}$ & & 289 & $44 \%$ & $46 \%$ \\
\hline & 33 & $X^{513}, X^{257}, X^{129}, X^{65}$ & & 273 & $47 \%$ & $50 \%$ \\
\hline & 17 & $X^{513}, X^{257}, X^{129}, X^{65}, X^{33}$ & & 265 & $48 \%$ & $53 \%$ \\
\hline & 9 & $X^{513}, X^{257}, X^{129}, X^{65}, X^{33}, X^{17}$ & & 261 & $49 \%$ & $54 \%$ \\
\hline & 5 & all odds excepted $X^{5}, X^{3}, X$ & & 259 & $49 \%$ & $56 \%$ \\
\hline & 3 & all odds excepted $X^{3}, X$ & & 258 & $50 \%$ & $57 \%$ \\
\hline & 1 & all odds excepted $X$ & \multirow[t]{2}{*}{5} & 257 & $50 \%$ & $58 \%$ \\
\hline & $-\infty$ & all odds & & 257 & $50 \%$ & $59 \%$ \\
\hline \multirow[t]{2}{*}{1024} & 1 & all odds excepted $X$ & \multirow[t]{2}{*}{5} & 512 & $0 \%$ & $0 \%$ \\
\hline & $-\infty$ & all odds & & 512 & $0 \%$ & $2 \%$ \\
\hline \multirow[t]{2}{*}{1026} & 1 & all odds excepted $X$ & \multirow[t]{2}{*}{6} & 513 & $0 \%$ & $-4 \%$ \\
\hline & $-\infty$ & all odds & & 513 & $0 \%$ & $-2 \%$ \\
\hline
\end{tabular}

gorithm 8 (Section 3.3). It does $\left(n-\left\lfloor\log _{2}(D)\right\rfloor\right)(D-1)(K-1)=O\left(n D \log _{2}(D)^{2}\right)$ field multiplications. This method can be improved with the trick from Section 3.4. To improve the performance, we compute the repeated squaring in-place. To do it, we allocate a buffer of $2 D-1$ coefficients in $\mathbb{F}_{2^{n}}$. The squaring and the modular reduction modify directly the current result.

Repeated squaring algorithm with multi-squaring tables. The authors of $\left[\mathrm{PCY}^{+} 15\right]$ propose to compute several squarings before reducing by $F$. Set $i$ this number of times. To compute $i$ squarings creates a result of degree $(D-1) 2^{i}$, but only the terms $X^{j 2^{i}}$ for $j \in\{0, \ldots, D-1\}$ are not null. To compute the reduction, firstly compute one time a table of $\left(X^{j 2^{i}} \bmod F\right)$ for $j \in\{0, \ldots, D-1\}$, then multiply each coefficient by the corresponding element in the table. The table is computed one time for all and is re-used for each modular reduction.

To create the table, we compute each $X^{j 2^{i}} \bmod F$ as $\left(X^{(j-1) 2^{i}} \bmod F\right) X^{2^{i}} \bmod F$. The multiplication by $X^{2^{i}}$ is just a shift of $2^{i}$, and all terms of degree strictly greater than $D-1$ are reduced with Algorithm 8 (by replacing $2 D-2$ by $D-1+2^{i}$ ). The table is useful only when $X^{j 2^{i}}$ is not already reduced by $F$, so when $2^{i} j \geq D$ and implies $j \geq\left\lceil\frac{D}{2^{i}}\right\rceil$. This table requires to store $\left(D-\left\lceil\frac{D}{2^{i}}\right\rceil\right) D$ elements of $\mathbb{F}_{2^{n}}$, and $D-\left\lceil\frac{D}{2^{i}}\right\rceil$ calls to Algorithm 8 are required to generate it, costing $O\left(2^{i}(K-1)\left(D-\left\lceil\frac{D}{2^{i}}\right\rceil\right)\right)$ field multiplications.

To compute $X^{2^{n}} \bmod F$, we take $X^{2^{\left.\log _{2}(D-1)\right\rfloor+i}} \bmod F$ from the table, then we compute $\left\lfloor\frac{n-i-\left\lfloor\log _{2}(D-1)\right\rfloor}{i}\right\rfloor$ steps of modular multi-squarings. Each step requires to raise $D$ elements of $\mathbb{F}_{2^{n}}$ at the power $2^{i}$, then to multiply each by the corresponding elements of the table. It costs $i D$ field squarings and $\left(D-\left[\frac{D}{2^{i}}\right\rceil\right) D$ field multiplications. To obtain $X^{2^{n}} \bmod F$, we terminate by $\left(\left(n-i-\left\lfloor\log _{2}(D-1)\right\rfloor\right) \bmod i\right)$ steps of modular squarings with the classical repeated squaring algorithm.

The final cost of this method is $\left(n-i-\left\lfloor\log _{2}(D-1)\right\rfloor\right) D=O(n D)$ field squarings and $\left(D-\left\lceil\frac{D}{2^{i}}\right\rceil\right)\left(2^{i}(K-1)+D\left\lfloor\frac{n-i-\left\lfloor\log _{2}(D-1)\right\rfloor}{i}\right\rfloor\right)+\left(\left(n-i-\left\lfloor\log _{2}(D-1)\right\rfloor\right) \bmod i\right)(D-1)(K-1)=$ $O\left(2^{i} D \log _{2}(D)^{2}+\frac{n}{i} D^{2}\right)$ field multiplications.

To choose the best algorithm for the Frobenius map, we just choose the one which minimizes the number of field multiplications. In practice, the repeated squaring algorithm is the best 
when approximately $D \geq n$, whereas the multi-squaring version is the best when $n>D$.

Table 12 summarizes the performance of both strategies for the Frobenius map, and compares our implementation to NTL and Magma. We use the Modexp function from Magma and the PlainFrobeniusMap function from NTL, both computing $X^{2^{n}} \bmod F$. We have studied also the strategy from Section 3.4 which permits to improve the Frobenius map by removing odd degree terms in the HFE polynomial. We choose $d=65$, which requires to remove one term when $D=130$ and three terms when $D=514$. The results confirm the theoretical speed-ups: MQsoft saves approximately $25 \%$ and $44 \%$ of computations by removing respectively one and three terms for the first strategy. Magma is also improved by this trick, probably because it uses also the classical Euclidean division, and does not compute multiplication by zero. It is not the case for NTL because it uses the fast Euclidean division [vzGG13, Algorithm 9.5].

The multi-squaring strategy is the fastest when $D$ is small compared to $n$. However, for $n=354$ and $D=129$, to set $d=65$ is enough to change the trade-off between both strategies. To remove odd degree terms is interesting for HFE-based NIST submissions which uses $D$ equals to 129 or more. However, this implies to increase by one the original parameters $(D=129$ and $D=513)$. Without modifying it, our best Frobenius map is 7 to 12 times faster than NTL.

Table 12: Number of mega cycles to compute the Frobenius map of a HFE polynomial. We use a Skylake processor (LaptopS).

\begin{tabular}{|l|l|l|l|l|l|l|}
\hline$n$ & $D$ & $d$ & Magma| & NTL & MQsoft (repeated squarings) & MQsoft (multi-squaring) \\
\hline \multirow{4}{*}{185} & 33 & 33 & 36.3 & 13.0 & 2.6 & $\mathbf{1 . 4}$ \\
\cline { 2 - 7 } & 129 & 129 & 169.3 & 136.5 & $\mathbf{1 7 . 2}$ & 18.1 \\
\cline { 2 - 7 } & 130 & 65 & 138.4 & 142.2 & $\mathbf{1 2 . 9}$ & 18.6 \\
\cline { 2 - 7 } & 513 & 513 & $1,000.8$ & $1,092.7$ & $\mathbf{1 0 8 . 9}$ & 223.7 \\
\cline { 2 - 7 } & 514 & 65 & 629.8 & $1,104.8$ & $\mathbf{5 9 . 0}$ & 223.0 \\
\hline 354 & 33 & 33 & 108.5 & 76.2 & 13.2 & $\mathbf{6 . 2}$ \\
\cline { 2 - 7 } & 129 & 129 & 559.0 & 551.0 & 93.3 & $\mathbf{7 9 . 9}$ \\
\cline { 2 - 7 } & 130 & 65 & 451.4 & 628.6 & $\mathbf{7 0 . 9}$ & 81.9 \\
\cline { 2 - 7 } & 513 & 513 & $3,371.0$ & $4,107.0$ & $\mathbf{5 6 6 . 0}$ & $1,061.5$ \\
\cline { 2 - 6 } & 514 & 65 & $2,019.0$ & $4,383.3$ & $\mathbf{3 1 2 . 1}$ & $1,053.6$ \\
\hline
\end{tabular}

\subsection{GCD in $\mathbb{F}_{2^{n}}[X]$}

Step 2 of Algorithm 6 requires to compute the GCD of two degree $D$ polynomials in $\mathbb{F}_{2^{n}}[X]$. The NTL library provides only the classical algorithm [vzGG13, Algorithm 3.5], which uses $O\left(D^{2}\right)$ field multiplications and $O(D)$ field inversions. We have implemented the half-GCD algorithm ([vzGG13, Algorithm 11.8],[BCG ${ }^{+} 17$, Algorithm 6.8]), which uses $\widetilde{O}(D)$ multiplications in $\mathbb{F}_{2^{n}}$. Our implementation of the half-GCD is based on the Karatsuba polynomial multiplication (Appendix C) and on the fast Euclidean division (Appendix D). These algorithms are implemented with constant-time arithmetic, but the GCD is in variable-time because the number of successive remainders is variable.

Table 13 compares the performance of GCD algorithms. Our classical GCD is three to four times better than NTL. This results of the difference of performance between our operations in $\mathbb{F}_{2^{n}}$. The half-GCD becomes faster only for a high degree (approximately 8193). 
Table 13: Number of mega cycles to compute the GCD in $\mathbb{F}_{2^{n}}[X]$ of a $D$ degree polynomial by a $D-1$ degree polynomial. We use a Skylake processor (LaptopS).

\begin{tabular}{|l|l|l|l|l|l|}
\hline$n$ & $D$ & Magma & NTL & MQsoft (gcd) & MQsoft (half-gcd) \\
\hline \multirow{4}{*}{185} & 33 & 2.2 & 0.620 & $\mathbf{0 . 1 2 1}$ & 0.535 \\
\cline { 2 - 6 } & 129 & 12.0 & 3.735 & $\mathbf{0 . 7 1 9}$ & 3.661 \\
\cline { 2 - 6 } & 513 & 129.9 & 39.8 & $\mathbf{7 . 6}$ & 30.2 \\
\cline { 2 - 6 } & 4097 & 3,060 & 2,057 & $\mathbf{4 1 9}$ & 726 \\
\cline { 2 - 6 } & 8193 & 9,485 & 8,131 & $\mathbf{1 , 8 3 8}$ & 2,168 \\
\cline { 2 - 6 } & 16385 & 27,168 & 32,175 & 7,304 & $\mathbf{6 , 4 0 7}$ \\
\hline 354 & 33 & 4.1 & 1.350 & $\mathbf{0 . 2 7 6}$ & 1.019 \\
\cline { 2 - 6 } & 129 & 23.0 & 7.973 & $\mathbf{1 . 8 7 0}$ & 7.303 \\
\cline { 2 - 6 } & 513 & 229.4 & 82.4 & $\mathbf{2 0 . 8}$ & 64.2 \\
\cline { 2 - 6 } & 4097 & 8,914 & 4,078 & $\mathbf{1 , 1 9 1}$ & 1,548 \\
\cline { 2 - 6 } & 8193 & 26,035 & 16,096 & 4,691 & $\mathbf{4 , 5 7 5}$ \\
\cline { 2 - 6 } & 16385 & 70,170 & 64,135 & 18,734 & $\mathbf{1 3 , 6 5 0}$ \\
\hline
\end{tabular}

\subsection{Performance of the Root Finding Algorithm in $\mathbb{F}_{2^{n}}[X]$}

Table 14 compares the best implementation of root finding of each library, and for the parameters of GeMSS and Gui. The results are similar to the performance of Frobenius map, which is the critical part of the root finding algorithm. MQsoft is five to nine times faster than NTL.

Table 14: Number of mega cycles to find the roots of a HFE polynomial. We use a Skylake processor (LaptopS).

\begin{tabular}{|l|l|l|l|l|l|}
\hline$n$ & $D$ & $d$ & Magma & NTL & MQsoft \\
\hline \multirow{2}{*}{174} & 513 & 513 & 1,130 & 1,089 & $\mathbf{1 1 6 . 0}$ \\
\cline { 2 - 6 } & 514 & 65 & 751.5 & 1,096 & $\mathbf{6 7 . 1}$ \\
\hline 185 & 33 & 33 & 39.0 & 14.0 & $\mathbf{1 . 7}$ \\
\hline 265 & 513 & 513 & 2,472 & 2,252 & $\mathbf{3 6 1 . 0}$ \\
\cline { 2 - 6 } & 514 & 65 & 1,559 & 2,304 & $\mathbf{2 0 3 . 3}$ \\
\hline \multirow{3}{*}{313} & 129 & 129 & 451.3 & 352.3 & $\mathbf{6 2 . 3}$ \\
\cline { 2 - 6 } & 130 & 65 & 356.7 & 388.5 & $\mathbf{5 3 . 1}$ \\
\hline \multirow{3}{*}{354} & 513 & 513 & 3,765 & 4,369 & $\mathbf{5 8 9 . 1}$ \\
\cline { 2 - 6 } & 514 & 65 & 2,341 & 4,392 & $\mathbf{3 2 9 . 2}$ \\
\hline \multirow{2}{*}{448} & 513 & 513 & 4,673 & 5,313 & $\mathbf{1 , 0 5 1 . 0}$ \\
\cline { 2 - 5 } & 514 & 65 & 2,821 & 5,464 & $\mathbf{5 8 1 . 5}$ \\
\hline
\end{tabular}

We have presented in this section the main algorithms that we have implemented in MQsoft to obtain an efficient root finding for HFE polynomials. However, our library proposes extra functions. The half-gcd requires to implement Karatsuba multiplication and fast Euclidean division, in both $\mathbb{F}_{2^{n}}[X]$. So, we propose a fast version of the root finding, based on a Frobenius map using the fast Euclidean division. This permits to have an efficient implementation of root finding for general applications, using a constant-time arithmetic in the base field. 


\section{Generation and Evaluation of the Public-key}

In this section, we study how implement efficiently important steps of the keypair generation and verifying process. Both are based on multivariate quadratic systems that we represent as quadratic forms.

\subsection{Generation of the Inner Secret-key Polynomial $f$}

For the set of completeness, we explain here the method used to compute $\mathbf{f}$ during the keypair generation (Section 1.2). It requires to compute $F\left(\sum_{k=1}^{n} \theta_{k} x_{k}, v_{1}, \ldots, v_{v}\right)$ (cf. Equation (3)). As a first step, we assume that $F$ does not have vinegar variables. We use two classical properties:

- The terms of degree strictly greater than 1 of $F$ can be represented as a quadratic form $\mathbf{X} Q \mathbf{X}^{t}$, where $\mathbf{X}=\left(X, X^{2}, X^{2^{2}}, X^{2^{3}}, \ldots, X^{2^{\left\lfloor\log _{2}(D)\right\rfloor}}\right)$ and $Q \in \mathcal{M}_{\left\lfloor\log _{2}(D)\right\rfloor+1}\left(\mathbb{F}_{2^{n}}\right)$ is upper triangular such that:

$$
Q_{i, j}= \begin{cases}B_{i+1} & \text { if } i=j \\ A_{j, i} & \text { if } i<j \\ 0 & \text { else. }\end{cases}
$$

We have the relation $F(X)=C+B_{0} X+\mathbf{X} Q \mathbf{X}^{t}$. In particular, $Q_{i, j}$ corresponds to the term $X^{2^{i}+2^{j}}$ of $F$.

- The linearity of the Frobenius implies $X^{2^{i}}=\left(\sum_{k=1}^{n} \theta_{k} x_{k}\right)^{2^{i}}=\sum_{k=1}^{n} \theta_{k}^{2^{i}} x_{k}$.

With these two properties, it is easy to verify that $\mathbf{X}=\mathbf{x} \Gamma$ where $\mathbf{x}=\left(x_{1}, \ldots, x_{n}\right)$ and $\Gamma \in \mathcal{M}_{n,\left\lfloor\log _{2}(D)\right\rfloor+1}\left(\mathbb{F}_{2^{n}}\right)$ is such that $\Gamma_{i, j}=\theta_{i+1}^{2^{j}}$. So, we deduce the multivariate quadratic form of $F$ :

$$
\mathbf{X} Q \mathbf{X}^{t}=\mathbf{x} Q^{\prime} \mathbf{x}^{t}, \text { with } Q^{\prime}=\Gamma Q \Gamma^{t} .
$$

We have $F=\mathbf{x} Q^{\prime} \mathbf{x}^{t}+B_{0} \theta \mathbf{x}^{t}+C$. In particular, $Q_{i, j}^{\prime}$ corresponds to the term $x_{i} x_{j}$ of $F$. Since $x_{i}^{2}=x_{i}$, we can add each $B_{0} \theta_{i}$ from the linear part to the term $Q_{i, i}^{\prime}$ of $Q^{\prime}$. We obtain:

$$
F=\mathbf{x} Q^{\prime \prime} \mathbf{x}^{t}+C, \text { with } Q^{\prime \prime} \text { such that } \mathbf{x} Q^{\prime \prime} \mathbf{x}^{t}=\left(\mathbf{x} Q^{\prime}+B_{0} \theta\right) \mathbf{x}^{t} .
$$

To simplify the computation of $\mathbf{f}, \theta$ (Section 1.2) is the same basis that the one used to represent an element of $\mathbb{F}_{2^{n}}$ (Section 1.6). In this way, $\mathbf{f}=\varphi\left(\mathbf{x} Q^{\prime \prime} \mathbf{x}^{t}+C\right.$ ) and we store $\varphi^{-1}(\mathbf{f})$. This is a monomial representation of $\mathbf{f}$.

To compute f, we compute first $\Gamma$ with $O\left(n \log _{2}(D)\right)$ field squarings. This matrix does not depend on $F$ and so it can be precomputed. Then, we compute $\Gamma \times Q$ with classical matrix product, requiring $O\left(n \log _{2}(D)^{2}\right)$ multiplications in $\mathbb{F}_{2}[X]$ and $O\left(n \log _{2}(D)\right)$ modular reductions. Finally, we multiply the previous result by $\Gamma^{t}$, requiring $O\left(n^{2} \log _{2}(D)\right)$ multiplications in $\mathbb{F}_{2}[X]$ and $O\left(n^{2}\right)$ modular reductions.

Now, we consider vinegar variables. $\gamma\left(v_{1}, \ldots, v_{v}\right)$ (Equation $\left.(1)\right)$ is quadratic in vinegar variables. We store it as a upper triangular matrix $W \in \mathcal{M}_{v}\left(\mathbb{F}_{2^{n}}\right)$ such that $\gamma\left(v_{1}, \ldots, v_{v}\right)=\mathbf{v} W \mathbf{v}^{t}+C$ where $\mathbf{v}=\left(v_{1}, \ldots, v_{v}\right)$ and $C \in \mathbb{F}_{2^{n}}$ is the constant term of $F$.

For the linear terms, we must compute $\sum_{i=0}^{\left\lfloor\log _{2}(D)\right\rfloor} \beta_{i} X^{2^{i}}=\mathbf{X} \beta^{t}$ with $\beta=\left(\beta_{0}, \ldots, \beta_{\left\lfloor\log _{2}(D)\right\rfloor}\right)$. Let $V \in \mathcal{M}_{\left\lfloor\log _{2}(D)\right\rfloor+1, v}\left(\mathbb{F}_{2^{n}}\right)$ and $\beta_{i}=\sum_{k=1}^{v} V_{i, k} v_{k}$. We have $\beta=\mathbf{v} V^{t}$, and so $\mathbf{X} \beta^{t}=$ $\mathbf{x} \Gamma V \mathbf{v}^{t} . \Gamma V$ is the matrix where the coefficient $(i, j)$ corresponds to the term $x_{i} v_{j}$. 
The final result is:

$$
\varphi^{-1}(\mathbf{f})=(\mathbf{x} \mathbf{v})\left(\begin{array}{cc}
Q^{\prime \prime} & \Gamma V \\
0 & W
\end{array}\right)(\mathbf{x} \mathbf{v})^{t}+C .
$$

The computation of $\Gamma V$ requires $O\left(n v \log _{2}(D)\right)$ multiplications in $\mathbb{F}_{2}[X]$ and $O(n v)$ modular reductions. So, the generation of the inner secret polynomials of $\mathbf{f}$ requires $O\left(n \log _{2}(D)\right)$ squarings in $\mathbb{F}_{2^{n}}, O\left(n \log _{2}(D)\left(n+v+\log _{2}(D)\right)\right.$ multiplications in $\mathbb{F}_{2}[X]$ and $O\left(n\left(n+v+\log _{2}(D)\right)\right)$ modular reductions.

We can notice that this representation of $\mathbf{f}$ permits easily to apply the linear change of variable by the matrix $\mathbf{S}$. Just replace $(\mathbf{x} \mathbf{v})$ by $(\mathbf{x} \mathbf{v}) \mathbf{S}$ and we obtain:

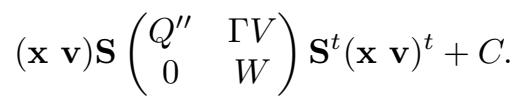

\subsection{Evaluation of Multivariate Quadratic Systems in $\mathbb{F}_{2}$}

The evaluation of the public-key is the main part of the verifying process (Section 1.4). It is iterated nb_ite times. Since the verification is a public process, it does not require to be protected against timing attacks. So, we can exploit the fact that for a random input, the evaluation of a monomial $x_{i} x_{j}$ in $\mathbb{F}_{2}$ has a probability of $75 \%$ to be null, and so to avoid $75 \%$ of computations. However, the evaluation in constant-time is required during the signature generation to evaluate the constant of the HFEv polynomial, which is quadratic in the vinegar variables. It is also used in other contexts, for example to encrypt a message for the HFE-based encryption scheme. In this section, we both study, variable-time and constant-time evaluation.

To evaluate the public-key $\mathbf{p}$, we can use different representations. The representation by equation consists to store the $m$ equations of $\mathbf{p}$ separately $\left(\mathbf{p} \in\left(\mathbb{F}_{2}\left[x_{1}, \ldots, x_{n+v}\right]\right)^{m}\right)$, whereas the representation by monomial consists to store the monomials of $\mathbf{p}$ separately $\left(\mathbf{p} \in \mathbb{F}_{2^{m}}\left[x_{1}, \ldots, x_{n+v}\right]\right.$, cf. Section 4.1).

The authors of $\left[\mathrm{CHR}^{+} 16\right]$ proposes a fast evaluation of the public-key with the representation by equation. In $\left[\mathrm{CLP}^{+} 18\right]$, the authors present a faster evaluation. To do so, they use a monomial representation of the public-key. Both, $\left[\mathrm{CHR}^{+} 16\right]$ and $\left[\mathrm{CLP}^{+} 18\right]$, have used the avx2 instructions set. We have chosen the monomial representation as in $\left[\mathrm{CLP}^{+} 18\right]$, because it exploits naturally the fact that on average, $75 \%$ of monomials are null.

Our variable-time evaluation uses only the classical method [BBG06]: we initialize an accumulator acc to the constant term of $\mathbf{p}$, and for each term $p_{i, j} x_{i} x_{j}$ with $p_{i, j} \in \mathbb{F}_{2^{m}}$, we add $p_{i, j}$ to acc only if $x_{i}=x_{j}=1$. This process is described in Algorithm 9 .

We have vectorized Algorithm 9. To do it, we just store acc with 256-bit registers, and we use 256-bit load, store and bitwise XOR to do vectorial computations. When $\left\lceil\frac{m}{64}\right\rceil$ is not a multiple of 4 , we sometimes add the use of 64-bit or 128-bit registers, to speed-up the implementation. Algorithm 9 is vulnerable to timing attacks, since the addition is done only if $x_{i}=x_{j}=1$. The traditional way to avoid this attack is to replace the conditional statement by a multiplication by $x_{i}$ (respectively $x_{j}$ ). But $x_{i}$ and $x_{j}$ are in $\mathbb{F}_{2}$, so the multiplication can be accelerated: it is equivalent to apply a mask which is the duplication of $x_{i}$ (respectively $x_{j}$ ) $m$ times. With this strategy, we obtain Algorithm 10.

To vectorize Algorithm 10, acc and acc_i are stored in 256-bit registers. However, the optimal choice to put each mask in a 256-bit register is not trivial. On the one hand, we can store 256-bit masks in the buffer mask. In this way, one load permits to create the 

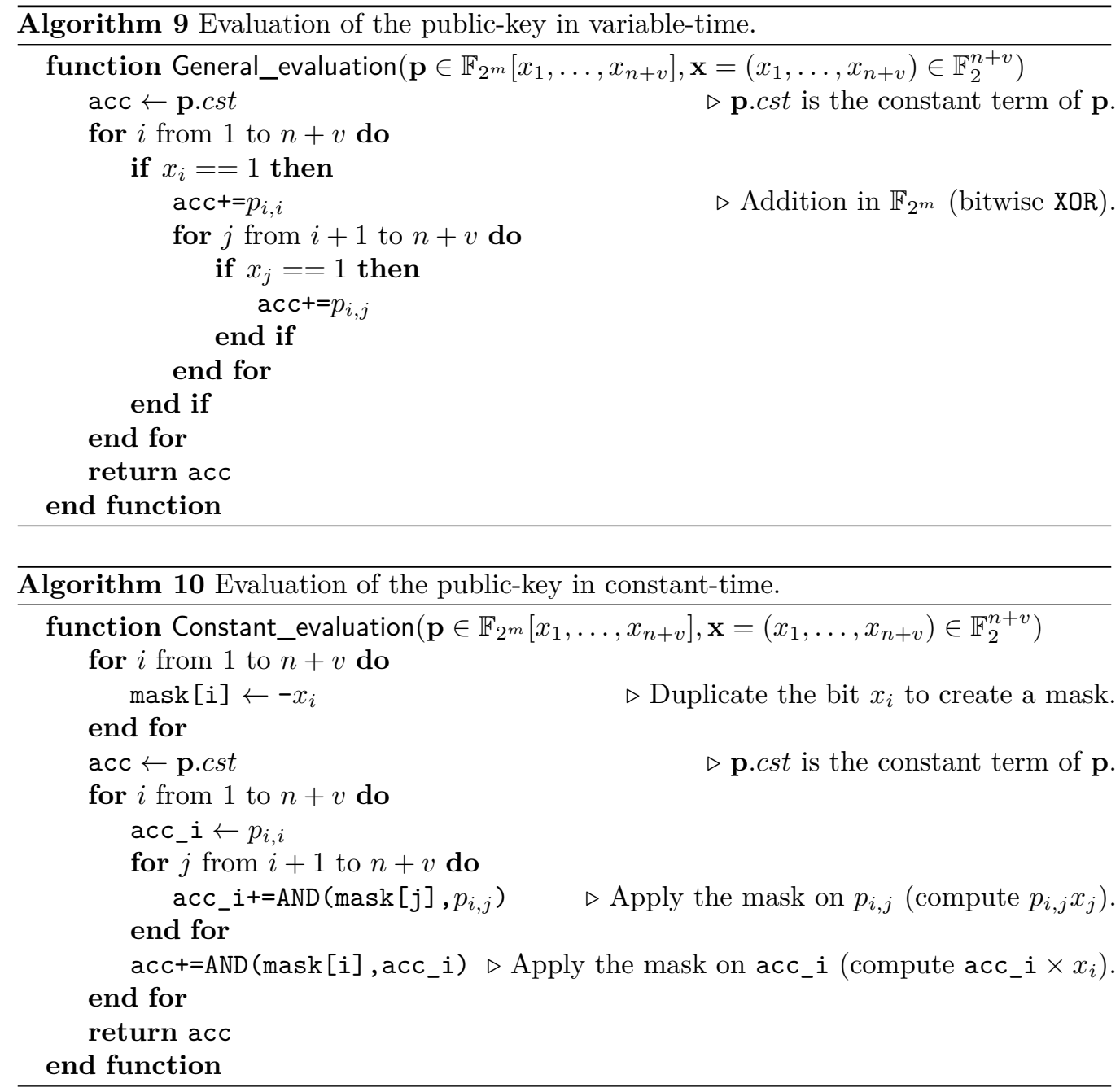

256-bit register. On the other hand, we can store 64 -bit masks in the buffer mask. The creation of the 256-bit register is done by one call to VPBROADCASTQ, which duplicates a 64-bit mask in a 256-bit register. This idea is described in Algorithm 11. We propose a new idea, described in Algorithm 12. Firstly, we unroll with a depth four the loop in $j$. Then, we store 64-bit masks in the buffer mask, but we load four 64-bit masks in one 256 -bit register. Then, to create a 256-bit mask from one of the four 64-bit masks, we use the VPERMQ instruction. It permits to create a 256-bit register where each 64-bit part is one of the four 64-bit part of the input. In particular, we use it to duplicate one 64-bit part of the input (which is a mask) in a 256-bit register. This method is the best: it requires only one load for four masks, unlike the two previous methods which require four loads (four 256-bit loads for the first method and four 64-bit loads for the second method).

Then, to apply the mask to $p_{i, j}$, we remark that the VPMASKMOVQ instruction permits to load data and to apply the mask in only one instruction. It permits to accelerate the evaluation.

More generally, this new method permits to improve the constant-time vector matrix product in $\mathbb{F}_{2}$, but is interesting only when the variable buffer is computed one time for several products using the same vector. We remark that this method is faster on Skylake processors, but on Haswell processors, the use of VPBROADCASTQ remains faster. 



Table 15 shows the performance of the evaluation that uses avx2. To improve the performance, we use the option -funroll-loops of gcc which unrolls loops to improve the use of the pipeline. The factor of performance between variable-time and constanttime implementation depends on $m$ : the factor is two for small values of $m$ and four for high values. The performance is affected by cache penalty when the public-key is too large. For $m=n+v=256$, we compare our code with the efficient implementation of $\left[\mathrm{CLP}^{+} 18\right]$, by using a similar processor (ServerH). We have similar times for constant-time implementation, and a speed-up of 1.38 for variable-time implementation. This speed-up is mainly dued to unrolled loops. Moreover, we have splitted the loop $i$ (respectively the loop $j$ ) in two loops with an Euclidean division by 64: the first is a loop for $i q \in \llbracket 0,\left\lfloor\frac{i}{64}\right\rfloor \rrbracket$, and the second is a loop for ir $\in \llbracket 0,63 \rrbracket$. In this way, for extracting $x_{i}$ which is the $i$-th bit from a vector of word, we take the $i r$-th bits of the $i q$-th word. It permits to simplify the extraction of bits from 64-bit variables.

For the constant-time evaluation, we have obtained our best times on Haswell by using Algorithm 11. However, on Skylake, Algorithm 12 is faster. For 256 equations and 256 variables (cf. Table 16), we obtain $61.4 \mathrm{Kc}$ with Algorithm 11 against $55.5 \mathrm{Kc}$ with Algorithm 12. Since Algorithm 11 is state-of-the-art on Haswell, we have obtained a new speeding record on Skylake, by a factor 1.1. For comparison, we obtain $23.2 \mathrm{Kc}$ for the variable-time evaluation.

For $m$ requiring one word (respectively two words), we use the 256-bit registers to do computations in $\mathbb{F}_{2^{m}}$ by pack of four elements (respectively two elements). This method implies to use masks to compute $q_{i, j} x_{j}$ for four (respectively two) successive values of $j$. To optimize the cases $m$ requiring one or two words is important because it permits to use a new strategy of parallelization: with $k$ cores, the public-key can be splitted in $k$ packets of 64 equations (respectively 128 equations), and each core can apply one time the evaluation for its part of the public-key. This method is interesting because the number of miss in the cache is decreased since each core has just a part of the public-key. In a general way, $m$ can be splitted in the way to use evaluation algorithms for smaller number of equations. 
Table 15: Number of kilo cycles to evaluate the public-key with MQsoft, for $m=n+v$. We use a Haswell processor (ServerH) with the avx2 instructions set. Turbo Boost is not used.

\begin{tabular}{|l|l|l|l|l|l|l|l|l|}
\hline category & $m=64$ & 128 & 192 & 256 & 320 & 384 & 448 & 512 \\
\hline constant-time & 2.01 & 14.1 & 44.7 & 89.1 & 196 & 318 & 478 & 853 \\
\hline variable-time & 1.15 & 6.46 & 17.1 & 37.3 & 74.5 & 120 & 191 & 205 \\
\hline
\end{tabular}

Table 16: Number of kilo cycles to evaluate the public-key with MQsoft, for $m=n+v$. We use a Skylake processor (DesktopS) with the avx2 instructions set. Turbo Boost is used.

\begin{tabular}{|l|l|l|l|l|l|l|l|l|}
\hline category & $m=64$ & 128 & 192 & 256 & 320 & 384 & 448 & 512 \\
\hline constant-time & 1.49 & 7.04 & 30.1 & 55.5 & 142 & 202 & 341 & 610 \\
\hline variable-time & 0.841 & 3.87 & 12.3 & 23.2 & 51.0 & 75.5 & 133 & 144 \\
\hline
\end{tabular}

Hybrid representation of the multivariate quadratic systems. When $m \bmod 64$ is small, the monomial representation of the public key is not optimal for the variable-time evaluation. The addition in $\mathbb{F}_{2^{m}}$ can be computed with 64 -bit, 128-bit and 256-bit XOR, so when $m$ mod 64 is small, many bits are unused during the computations. In memory, we use $\left\lceil\frac{m}{64}\right\rceil$ words to store each monomial. The unused bits are set to zero. When $m$ mod 64 is small, to store the $m$ mod 64 last equations separately is more efficient. This permits to obtain an optimal representation for a large part of equations, then to optimize the representation of the last remaining equations. We have applied this idea to GeMSS256, for which $m$ is 324 and so $m \bmod 64$ is equal to 4 . The 320 first equations are stored by using the monomial representation, whereas the four last equations are stored one-by-one. With this method, we save $18 \%$ of the pratical size of the public key, and we obtain a slight speed-up of $5 \%$ during the verifying process.

\section{Performance of GeMSS, Gui and DualModeMS}

In this section, we show the speed-ups obtained for GeMSS, Gui and DualModeMS thanks to MQsoft. The difference between Gui and GeMSS are explained in Section 1.5. MQsoft uses the SHA-3 function from the Keccak Code Package [GBA13] and the SHA-2 function from OpenSSL. Random elements ar generated by the determinist random bytes generator provided by the NIST during the competition (which is based on AES from OpenSSL). The original implementation of GeMSS requires NTL library to compute the root finding. In MQsoft, NTL has been completely removed. DualModeMS is based on GeMSS implementation and so is naturally supported by MQsoft.

\subsection{Performance of NIST Implementations}

Table 17 summarizes the performance measurements of GeMSS additional (best) implementation and Gui PCLMULQDQ implementation which have been submitted to NIST PQC standardization process. The measurements of GeMSS have been corrected since the submission. The parameter $D$ had been set by error at 512 in the implementation (and during the measurements). Because the Frobenius map has not been implemented in constant-time, $D=512$ allows to save $27 \%$ of computations in the critical part of the signature generation (cf. Table 11). As explained in Section 1.5, we have removed the EUF-CMA security property of Gui in the PCLMULQDQ implementation, because this property is not available in GeMSS original implementation. We have also added the 
performance of DualModeMS, which will be studied in Section 5.5. Measurements more detailed are available in Appendix E.

Table 17: Number of mega cycles (Mc) for each cryptographic operation with a Haswell processor (DesktopH), followed by the speed-up between Haswell and Skylake processors (DesktopH versus DesktopS). For example, $110 \quad S:+21 \%$ means a performance of 110 Mc on Haswell, and a performance of $\frac{110}{1.21}=91 \mathrm{Mc}$ on Skylake.

\begin{tabular}{|c|c|cc|cc|cc|}
\hline scheme & $(n, D, \Delta, v, \mathrm{nb}$ _ite $)$ & \multicolumn{2}{|c|}{ key gen. } & \multicolumn{2}{|c|}{ signature gen. } & \multicolumn{2}{c|}{ signature verif. } \\
\hline GeMSS128 & $(174,513,12,12,4)$ & 110 & $S:+21 \%$ & 1400 & $S:+39 \%$ & 0.14 & $S:+15 \%$ \\
\hline GeMSS192 & $(265,513,22,20,4)$ & 510 & $S:+19 \%$ & 3500 & $S:+35 \%$ & 0.39 & $S:+13 \%$ \\
\hline GeMSS256 & $(354,513,30,33,4)$ & 1500 & $S:+27 \%$ & 6600 & $S:+63 \%$ & 0.91 & $S:+3.2 \%$ \\
\hline Gui-184 & $(184,33,16,16,4)$ & 670 & $S:+9.5 \%$ & 31 & $S:+38 \%$ & 0.16 & $S:+13 \%$ \\
\hline Gui-312 & $(312,129,24,20,2)$ & 4700 & $S:+13 \%$ & 450 & $S:+40 \%$ & 0.25 & $S:+7.2 \%$ \\
\hline Gui-448 & $(448,513,32,28,2)$ & 27000 & $S:+3.4 \%$ & 16000 & $S:+45 \%$ & 0.65 & $S:+6.7 \%$ \\
\hline Inner.DualModeMS128 & $(266,129,10,11,1)$ & 410 & $S:+16 \%$ & 130 & $S:+34 \%$ & 0.083 & $S:+9.9 \%$ \\
\hline DualModeMS128 & $(266,129,10,11,1)$ & 1700000 & $S:+5.3 \%$ & 8800 & $S:+34 \%$ & 8.2 & $S:+5.7 \%$ \\
\hline
\end{tabular}

The main difference between Haswell and Skylake processors is the performance of the PCLMULQDQ instruction (cf. Table 4). This impacts mainly the performance of the signature generation, but also the one of the keypair generation.

\subsection{Performance of MQsoft}

We present in Tables 18 and 19 new performance results for GeMSS and Gui. More detailed measurements are available in Appendix F. The choice of the parameter $n$ in Gui requires, for all levels of security, to choose irreducible pentanomials to define $\mathbb{F}_{2^{n}}$. This affects the performance in comparison to GeMSS which uses only irreducible trinomials. For this reason, we propose to sligthly increase the parameter $n$ in Gui to use trinomials. This choice does not change the security. For $n=184$ and $n=312$, we propose respectively to take $n=185$ and $n=313$. However, for $n=448$, we keep this value since $n=449$ increases the cost of the multiplication in $\mathbb{F}_{2^{n}}$. Indeed, the cost of such multiplication is a function of $\left\lceil\frac{n}{64}\right\rceil$ (Section 2.2). In practice, the impact of the modular reduction is important for small values of $n$ (as $n=185$ ). The obtained speed-up decreases when $n$ increases, because the multiplication is asymptotically coster than the modular reduction.

Table 18: Number of mega cycles (Mc) for each cryptographic operation with our library for a Haswell processor (DesktopH), followed by the speed-up between the best implementation provided for the NIST submissions versus our implementation. For example, $40 \quad \delta:+180 \%$ means a performance of $40 \mathrm{Mc}$ with MQsoft, and a performance of $40 \times 2.8=110 \mathrm{Mc}$ for the NIST implementations.

\begin{tabular}{|c|c|cc|cc|c|c|}
\hline scheme & $(n, D, \Delta, v, \mathrm{nb}$ _ite $)$ & \multicolumn{2}{|c|}{ key gen. } & \multicolumn{2}{|c|}{ signature gen. } & \multicolumn{2}{c|}{ signature verif. } \\
\hline GeMSS128 & $(174,513,12,12,4)$ & 40 & $\delta:+180 \%$ & 860 & $\delta:+57 \%$ & 0.072 & $\delta:+100 \%$ \\
\hline GeMSS192 & $(265,513,22,20,4)$ & 210 & $\delta:+140 \%$ & 2800 & $\delta:+25 \%$ & 0.21 & $\delta:+84 \%$ \\
\hline GeMSS256 & $(354,513,30,33,4)$ & 620 & $\delta:+130 \%$ & 5300 & $\delta:+23 \%$ & 0.52 & $\delta:+76 \%$ \\
\hline Gui-184 & $(184,33,16,16,4)$ & 51 & $\delta:+1200 \%$ & 22 & $\delta:+39 \%$ & 0.088 & $\delta:+81 \%$ \\
\hline Gui-185 & $(185,33,16,16,4)$ & 52 & $\delta:+1200 \%$ & 18 & $\delta:+72 \%$ & 0.09 & $\delta:+77 \%$ \\
\hline Gui-312 & $(312,129,24,20,2)$ & 340 & $\delta:+1300 \%$ & 400 & $\delta:+13 \%$ & 0.15 & $\delta:+64 \%$ \\
\hline Gui-313 & $(313,129,24,20,2)$ & 340 & $\delta:+1300 \%$ & 370 & $\delta:+21 \%$ & 0.15 & $\delta:+61 \%$ \\
\hline Gui-448 & $(448,513,32,28,2)$ & 1400 & $\delta:+1800 \%$ & 6400 & $\delta:+150 \%$ & 0.41 & $\delta:+60 \%$ \\
\hline Inner.DualModeMS128 & $(266,129,10,11,1)$ & 190 & $\delta:+120 \%$ & 100 & $\delta:+30 \%$ & 0.04 & $\delta:+110 \%$ \\
\hline DualModeMS128 & $(266,129,10,11,1)$ & 1700000 & $\delta:+0 \%$ & 6600 & $\delta:+33 \%$ & 8.2 & $\delta:+0 \%$ \\
\hline
\end{tabular}


Table 19: Number of mega cycles (Mc) for each cryptographic operation with our library for a Skylake processor (DesktopS), followed by the speed-up between the best implementation provided for the NIST submissions versus our implementation. For example, $36 \delta:+160 \%$ means a performance of 36 Mc with MQsoft, and a performance of $36 \times 2.6=94 \mathrm{Mc}$ for the NIST implementations.

\begin{tabular}{|c|c|c|c|c|}
\hline scheme & $\left(n, D, \Delta, v, \mathrm{nb} \_\right.$ite $)$ & key gen. & signature gen. & signature verif. \\
\hline GeMSS128 & $(174,513,12,12,4)$ & $36 \quad \delta:+160 \%$ & $600 \delta:+64 \%$ & $0.067 \delta:+88 \%$ \\
\hline GeMSS192 & $(265,513,22,20,4)$ & $190 \delta:+120 \%$ & $1900 \quad \delta:+37 \%$ & $0.2 \quad \delta:+78 \%$ \\
\hline GeMSS256 & $(354,513,30,33,4)$ & $560 \quad \delta:+100 \%$ & $3100 \quad \delta:+30 \%$ & $0.45 \quad \delta:+96 \%$ \\
\hline Gui-184 & $(184,33,16,16,4)$ & $49 \quad \delta:+1200 \%$ & $17 \delta:+32 \%$ & $0.081 \quad \delta:+74 \%$ \\
\hline Gui-185 & $(185,33,16,16,4)$ & $49 \delta:+1100 \%$ & $13 \delta:+68 \%$ & $0.081 \delta:+75 \%$ \\
\hline Gui-312 & $(312,129,24,20,2)$ & $310 \quad \delta:+1200 \%$ & $290 \quad \delta:+13 \%$ & $0.13 \delta:+85 \%$ \\
\hline Gui-313 & $(313,129,24,20,2)$ & $310 \quad \delta:+1200 \%$ & $260 \quad \delta:+24 \%$ & $0.13 \quad \delta:+83 \%$ \\
\hline Gui-448 & $(448,513,32,28,2)$ & $1300 \quad \delta:+1900 \%$ & $4400 \quad \delta:+150 \%$ & $0.33 \quad \delta:+87 \%$ \\
\hline Inner.DualModeMS128 & $(266,129,10,11,1)$ & $170 \delta:+100 \%$ & $69 \delta:+43 \%$ & $0.03 \delta:+150 \%$ \\
\hline DualModeMS128 & $(266,129,10,11,1)$ & $1600000 \quad \delta:+0 \%$ & $4600 \quad \delta:+43 \%$ & $7.8 \quad \delta:+0 \%$ \\
\hline
\end{tabular}

Table 18 and 19 summarize also the speed-up between NIST submissions and MQsoft. For GeMSS, the main part of the implementation which was in variable-time has been modified to be in constant-time. To obtain these results, we have improved the complexity of the generation of $\mathbf{f}$ by using the method explained in Section 4.1, and we have vectorized the multiplication by $\mathbf{T}$ (cf. Section 5.4). We have improved the arithmetic in $\mathbb{F}_{2^{n}}$ with a better multiplication, a vectorial modular reduction and an inverse computed with ITMIA. In the first implementation, the GCD was computed by NTL. With our efficient computation of the inverse in $\mathbb{F}_{2^{n}}^{\times}$, the GCD becomes negligible compared to the Frobenius map. The verifying process is accelerated by a factor between 1.7 and 2, thanks to our vectorial implementation of the evaluation of the public-key.

For our implementation of Gui, we obtain at least a speed-up of a factor 12 for the keypair generation, probably because the original implementation uses evaluations of $F$ then an interpolation to compute $\mathbf{f}$. The signature generation is faster by a factor between 1.2 and 2.5, thanks to our efficient implementation of the arithmetic in $\mathbb{F}_{2^{n}}$. We target the Skylake processors, whereas the original implementation targets the Haswell processors. For this reason, the speed-up should be less advantageous on the Haswell processors. However, the modular reduction of the NIST submission uses PCLMULQDQ. This method is probably slower on Haswell than the shift-and-add strategy. Moreover, the original implementation uses multi-squaring tables to compute the Frobenius map (Section 3.5). For the 256-bit level of security, this is inefficient compared to the classical repeated squaring algorithm (cf. Table 12 for $D=513$ ). But this error has been corrected in libpqcrypto ${ }^{4}$, and so in SUPERCOP $^{5}$ (SUPERCOP uses the implementation of libpqcrypto). The verifying process is faster by a factor between 1.6 and 1.9 .

\subsection{Statistics on the Performance of GeMSS and Gui}

We propose here an other method to evaluate the performance of GeMSS and Gui. The experimental protocol is the following. Firstly, we measure the performance of a small number of keypair generation (between 10 and 100). The set of the measurements will permit to compute the average, the standard deviation, the median as well as the first and third quartiles. Secondly, for three keypairs, we measure the performance of the signing and verifying processes on a small number of documents (between 256 and 2048). To do this, we set the length of each document to 59 bytes, then we generate randomly a

\footnotetext{
${ }^{4}$ https://libpqcrypto.org

${ }^{5}$ https://bench.cr.yp.to/supercop.html
} 
small number of documents, stored in a buffer. Then, we measure the performance of the signing process on these documents, and each signature is stored in a second buffer. Finally, we use this buffer to measure the performance of the verifying process. The code was compiled with gcc -04 -mavx2 -mpclmul -mpopcnt -funroll-loops. Here, we use the version 6.5 .0 of gcc. In Table 20, we present the results for the 128-bit security level, with three significant digits. The complete table is on our website [MQs18].

Table 20: Statistics in mega cycles (Mc) for each cryptographic operation, for a Skylake process (LaptopS). For three different keypairs, documents of length 59 bytes are signed then verified. The EUF-CMA property is implemented for all schemes.

\begin{tabular}{|c|c|c|c|c|c|c|}
\hline scheme & operation & average & std. deviation & first quartile & median & third quartile \\
\hline \multirow{7}{*}{$\begin{array}{c}\text { Gui-184 } \\
\text { (libpqcrypto) }\end{array}$} & keygen & 771 & 9.62 & 764 & 769 & 776 \\
\hline & sign 1 & 215 & 201 & 66.8 & 154 & 301 \\
\hline & $\operatorname{sign} 2$ & 225 & 217 & 69.4 & 162 & 303 \\
\hline & $\operatorname{sign} 3$ & 214 & 211 & 67.3 & 147 & 288 \\
\hline & verify 1 & 0.298 & 0.0281 & 0.285 & 0.292 & 0.3 \\
\hline & verify 2 & 0.295 & 0.02 & 0.285 & 0.292 & 0.299 \\
\hline & verify 3 & 0.296 & 0.0222 & 0.285 & 0.292 & 0.299 \\
\hline \multirow{7}{*}{$\begin{array}{l}\text { Gui-184 } \\
\text { (MQsoft) }\end{array}$} & keygen & 59.5 & 4.1 & 56.4 & 58.4 & 62 \\
\hline & sign 1 & 153 & 152 & 44.2 & 107 & 208 \\
\hline & $\operatorname{sign} 2$ & 155 & 146 & 49.7 & 109 & 219 \\
\hline & $\operatorname{sign} 3$ & 154 & 151 & 50 & 108 & 208 \\
\hline & verify 1 & 0.207 & 0.0188 & 0.193 & 0.205 & 0.218 \\
\hline & verify 2 & 0.206 & 0.0196 & 0.192 & 0.204 & 0.217 \\
\hline & verify 3 & 0.207 & 0.0337 & 0.192 & 0.204 & 0.217 \\
\hline \multirow{7}{*}{$\begin{array}{l}\text { Gui-185 } \\
\text { (MQsoft) }\end{array}$} & keygen & 59.4 & 3.46 & 56.5 & 59.1 & 60.7 \\
\hline & $\operatorname{sign} 1$ & 120 & 115 & 38.8 & 86 & 165 \\
\hline & $\operatorname{sign} 2$ & 125 & 123 & 38.7 & 87.4 & 165 \\
\hline & $\operatorname{sign} 3$ & 124 & 120 & 40.1 & 88.9 & 172 \\
\hline & verify 1 & 0.209 & 0.0199 & 0.195 & 0.207 & 0.22 \\
\hline & verify 2 & 0.21 & 0.0208 & 0.195 & 0.208 & 0.221 \\
\hline & verify 3 & 0.21 & 0.0211 & 0.195 & 0.208 & 0.221 \\
\hline \multirow{7}{*}{$\begin{array}{l}\text { GeMSS128 } \\
\text { (MQsoft) }\end{array}$} & keygen & 45.5 & 1.02 & 45.2 & 45.3 & 45.3 \\
\hline & sign 1 & 1670 & 1340 & 694 & 1260 & 2180 \\
\hline & $\operatorname{sign} 2$ & 1700 & 1400 & 697 & 1280 & 2210 \\
\hline & $\operatorname{sign} 3$ & 1640 & 1330 & 689 & 1260 & 2070 \\
\hline & verify 1 & 0.182 & 0.0168 & 0.17 & 0.18 & 0.191 \\
\hline & verify 2 & 0.182 & 0.0164 & 0.17 & 0.18 & 0.19 \\
\hline & verify 3 & 0.182 & 0.017 & 0.17 & 0.18 & 0.191 \\
\hline
\end{tabular}

In this section, we only measure the Gui implementation from libpqcrypto. The main difference with the original implementation is the correction of a mistake in the way to compute the Frobenius map (as explained in the previous section). This change impacts only the performance of the signing process of Gui-448.

Then, we have updated MQsoft to achieve the EUF-CMA security property in order to be comparable to libpqcrypto. The EUF-CMA version implies a slow down of the signing process. We do not measure the performance of the NIST submission of GeMSS because it does not achieve this property.

In Table 20, we remark that the timings for the signing process are really unstable. This is explained by the fact that during Algorithm 2, the root finding algorithm is reiterated when any root is found. The verifying process is slightly unstable, since the evaluation of the public-key is implemented in variable-time. 
SUPERCOP benchmarks. We have integrated MQsoft in SUPERCOP. The results are summarized in Table 21. Here again, we have benchmarked the EUF-CMA versions, and we compare MQsoft to the Gui implementation of SUPERCOP (which is identical to libpqcrypto). We give only the measurements to sign and verify a document of length 59 bytes. The complete table is on our website [MQs18].

Table 21: Median in mega cycles (Mc) for each cryptographic operation, for a Skylake process (LaptopS). We compare the Gui implementation from SUPERCOP (S) with MQsoft. For three different keypairs, documents of length 59 bytes are signed then verified. The EUF-CMA property is implemented for all schemes.

\begin{tabular}{|c|c|c|c|c|c|c|c|c|c|}
\hline scheme & keygen 1 & keygen 2 & keygen 3 & sign 1 & sign 2 & sign 3 & verify 1 & verify 2 & verify 3 \\
\hline Gui-184 (S) & 485 & 487 & 488 & 208 & 178 & 130 & 0.279 & 0.273 & 0.285 \\
\hline Gui-184 & 53.7 & 54 & 53.7 & 73.8 & 122 & 129 & 0.101 & 0.0998 & 0.107 \\
\hline Gui-185 & 55.2 & 54.7 & 54.7 & 105 & 102 & 132 & 0.107 & 0.102 & 0.107 \\
\hline GeMSS128 & 44.8 & 44.8 & 44.7 & 1380 & 1390 & 1270 & 0.0893 & 0.0961 & 0.088 \\
\hline
\end{tabular}

In this section, we give the results for Skylake processors. We have also made similar experiments on Haswell and obtained similar ratios. As in the previous section, the signing process is impacted by the performance of PCLMULQDQ (Table 4).

\subsection{Performance of Keypair Generation}

Table 22 summarizes the time of most important steps of the keypair generation (Section 1.2). These steps are achieved in constant-time. The generation of $\mathbf{f}$ is computed as explained in Section 4.1. We have vectorized the multiplication by $\mathbf{T}$, which is based on a vector matrix product in $\mathbb{F}_{2}$ implemented with avx2 instructions set. For the moment, the multiplication by $\mathbf{S}$ is not vectorized. This is the crucial part of the keypair generation when PCLMULQDQ is available. For the multiplication by $\mathbf{T}$, we have obtained approximately a factor two with the vectorization. So, we can hope to obtain the same factor for the multiplication by $\mathbf{S}$.

Table 22: Number of mega cycles for main steps of keypair generations with MQsoft. We use a Skylake processor (DesktopS).

\begin{tabular}{|c|c|c|c|c|c|c|}
\hline scheme & sec. level & $(n, D, \Delta, v)$ & gen. f & apply S & apply T & keypair gen. \\
\hline \multirow{3}{*}{ GeMSS } & 128 & $(174,513,12,12)$ & 4.04 & 26.5 & 5.78 & 36.2 \\
\cline { 2 - 7 } & 192 & $(265,513,22,20)$ & 21.0 & 144 & 20.2 & 189 \\
\cline { 2 - 7 } & 256 & $(354,513,30,33)$ & 49.6 & 446 & 61.9 & 564 \\
\hline \multirow{3}{*}{ Gui } & 128 & $(184,33,16,16)$ & 2.73 & 33.1 & 7.11 & 48.6 \\
\cline { 2 - 7 } & & $(185,33,16,16)$ & 2.57 & 33.6 & 7.16 & 48.9 \\
\cline { 2 - 7 } & \multirow{2}{*}{192} & $(312,129,24,20)$ & 22.7 & 229 & 39.6 & 307 \\
\cline { 2 - 7 } & & $(313,129,24,20)$ & 22.7 & 231 & 40.3 & 310 \\
\cline { 2 - 7 } & 256 & $(448,513,32,28)$ & 100 & 1080 & 120 & 1340 \\
\hline
\end{tabular}

\subsection{Performance of DualModeMS}

DualModeMS [FPR17] scheme is a candidate to NIST PQC standardization process. It is composed by two distinct layers. The first one (Inner.DualModeMS) is a re-parametrization of GeMSS. The second one (DualModeMS) is a modified HFE-based signature scheme 
which permits to decrease the size of the public-key, but by increasing the size of the signature. These parameters are chosen to minimize the sum of both sizes. Since the NIST submission DualModeMS is based on the GeMSS implementation, MQsoft supports naturally DualModeMS. The original implementation supported only the 128-bit level of security. For Inner.DualModeMS, we obtain the original implementation by modifying only the security parameters in the GeMSS128 implementation. Our new library permits to support the three levels of security. Table 23 and 24 compare the performance of DualModeMS between the additional implementation of the NIST submission and MQsoft. The main part of signature generation is to compute a large number (between 64 and 256) of HFE-based signatures with Inner.DualModeMS. For a 128-bit level of security, MQsoft permits to obtain a factor 1.33 on Haswell and 1.43 on Skylake (cf. Table 18 and 19). This is directly obtained by the improvement of Inner.DualModeMS. These factors are mainly obtained by using the multi-squaring tables to compute the Frobenius map (Section 3.5). The original implementation used the classical repeated squaring algorithm.

Table 23: Number of mega cycles for each cryptographic operation for DualModeMS. We use a Haswell processor (DesktopH).

\begin{tabular}{|c|c|c|c|c|}
\hline scheme & $\left(n, D, \Delta, v, n b \_\right.$ite $)$ & key gen. & sign. gen. & sign. verif. \\
\hline Inner.DualModeMS128 NIST & $(266,129,10,11,1)$ & 410 & 130 & 0.083 \\
\hline Inner.DualModeMS128 & $(266,129,10,11,1)$ & 190 & 100 & 0.04 \\
\hline Inner.DualModeMS192 & $(402,129,18,18,1)$ & 930 & 220 & 0.13 \\
\hline Inner.DualModeMS256 & $(544,129,32,32,1)$ & 3600 & 440 & 0.24 \\
\hline DualModeMS128 NIST & $(266,129,10,11,1)$ & $1.7 \mathrm{M}$ & 8800 & 8.2 \\
\hline DualModeMS128 & $(266,129,10,11,1)$ & $1.7 \mathrm{M}$ & 6600 & 8.2 \\
\hline DualModeMS192 & $(402,129,18,18,1)$ & $6.4 \mathrm{M}$ & 22000 & 15 \\
\hline DualModeMS256 & $(544,129,32,32,1)$ & $16 \mathrm{M}$ & 120000 & 26 \\
\hline
\end{tabular}

Table 24: Number of mega cycles for each cryptographic operation for DualModeMS. We use a Skylake processor (DesktopS).

\begin{tabular}{|c|c|c|c|c|}
\hline scheme & $\left(n, D, \Delta, v, \mathrm{nb} \_\right.$ite $)$ & key gen. & sign. gen. & sign. verif. \\
\hline Inner.DualModeMS128 NIST & $(266,129,10,11,1)$ & 350 & 98 & 0.076 \\
\hline Inner.DualModeMS128 & $(266,129,10,11,1)$ & 170 & 69 & 0.03 \\
\hline Inner.DualModeMS192 & $(402,129,18,18,1)$ & 850 & 150 & 0.098 \\
\hline Inner.DualModeMS256 & $(544,129,32,32,1)$ & 3400 & 290 & 0.2 \\
\hline DualModeMS128 NIST & $(266,129,10,11,1)$ & $1.6 \mathrm{M}$ & 6500 & 7.8 \\
\hline DualModeMS128 & $(266,129,10,11,1)$ & $1.6 \mathrm{M}$ & 4600 & 7.8 \\
\hline DualModeMS192 & $(402,129,18,18,1)$ & $5.7 \mathrm{M}$ & 16000 & 15 \\
\hline DualModeMS256 & $(544,129,32,32,1)$ & $14 \mathrm{M}$ & 81000 & 25 \\
\hline
\end{tabular}

\section{Conclusion}

MQsoft is an efficient library to do HFE-based multivariate cryptography. We obtain interesting speed-ups for GeMSS, Gui and for the signature generation of DualModeMS. Our library provides an efficient constant-time arithmetic, which is on average four times faster than NTL. We have proposed a new method to improve the root finding for specific HFE polynomials, but the security analysis must be studied in depth. We have exploited 
the architecture to obtain efficient implementations for the evaluation of multivariate quadratic systems in $\mathbb{F}_{2}$.

However, our library can be improved again. In fact, the generation of keypair is not completely vectorized. The library is in constant-time for a large part, but is not completely protected against timing attacks. The generation and inversion of random invertible matrices require to use constant-time Gaussian elimination [BCS13]. It is not implemented in MQsoft for the moment. Moreover, the GCD is implemented in variable-time since the number of iterations is variable. To solve this problem, the Gui submission proposes a constant-time implementation which returns a correct GCD only if its degree is one.

During the review process, GeMSS was selected in the second round of the NIST PQC standardization process. MQsoft will permit to improve the performance of GeMSS, as well as to propose other trade-offs between security and performance.

\section{Acknowledgements}

We thank the reviewers for their very useful comments, and Peter Schwabe for the time spent to confirm the performance of MQsoft. This work was financially supported by the French Ministère des armées - Direction Générale de l'Armement, and prepared with the support of the French Programme d'Investissement d'Avenir under national project RISQ ${ }^{6}$ $\mathrm{P} 141580$.

\section{References}

[ALH10a] Diego F. Aranha, Julio López, and Darrel Hankerson. Efficient software implementation of binary field arithmetic using vector instruction sets. In Michel Abdalla and Paulo S. L. M. Barreto, editors, Progress in Cryptology - LATINCRYPT 2010, First International Conference on Cryptology and Information Security in Latin America, Puebla, Mexico, August 8-11, 2010 , Proceedings, volume 6212 of Lecture Notes in Computer Science, pages 144161. Springer, 2010.

[ALH10b] Diego F. Aranha, Julio López, and Darrel Hankerson. High-speed parallel software implementation of the $\eta \mathrm{t}$ pairing. In Josef Pieprzyk, editor, Topics in Cryptology - CT-RSA 2010, The Cryptographers' Track at the RSA Conference 2010, San Francisco, CA, USA, March 1-5, 2010. Proceedings, volume 5985 of Lecture Notes in Computer Science, pages 89-105. Springer, 2010.

[BBG06] Côme Berbain, Olivier Billet, and Henri Gilbert. Efficient implementations of multivariate quadratic systems. In Selected Areas in Cryptography, 13th International Workshop, SAC 2006, Montreal, Canada, August 17-18, 2006 Revised Selected Papers, pages 174-187, 2006.

[BCG $\left.{ }^{+} 17\right]$ Alin Bostan, Frédéric Chyzak, Marc Giusti, Romain Lebreton, Grégoire Lecerf, Bruno Salvy, and Éric Schost. Algorithmes efficaces en calcul formel, August 2017. 686 pages. Édition 1.0.

[BCM94] Wieb Bosma, John J. Cannon, and Graham Matthews. Programming with algebraic structures: Design of the MAGMA language. In Proceedings of the International Symposium on Symbolic and Algebraic Computation, ISSAC '94, Oxford, UK, July 20-22, 1994, pages 52-57, 1994.

\footnotetext{
${ }^{6}$ https://risq.fr/?page_id=31\&lang=en
} 
[BCR13] Jingguo Bi, Qi Cheng, and J. Maurice Rojas. Sub-linear root detection, and new hardness results, for sparse polynomials over finite fields. In Manuel Kauers, editor, International Symposium on Symbolic and Algebraic Computation, ISSAC'13, Boston, MA, USA, June 26-29, 2013, pages 61-68. ACM, 2013 .

[BCS13] Daniel J. Bernstein, Tung Chou, and Peter Schwabe. Mcbits: Fast constanttime code-based cryptography. In Guido Bertoni and Jean-Sébastien Coron, editors, Cryptographic Hardware and Embedded Systems - CHES 2013 15th International Workshop, Santa Barbara, CA, USA, August 20-23, 2013. Proceedings, volume 8086 of Lecture Notes in Computer Science, pages 250272. Springer, 2013.

[BFP13] Luk Bettale, Jean-Charles Faugère, and Ludovic Perret. Cryptanalysis of hfe, multi-hfe and variants for odd and even characteristic. Des. Codes Cryptography, 69(1):1-52, 2013.

[BFS04] Magali Bardet, Jean-Charles Faugère, and Bruno Salvy. On the complexity of Gröbner basis computation of semi-regular overdetermined algebraic equations. In International Conference on Polynomial System Solving - ICPSS, pages $71-75,2004$.

[BFSY05] Magali Bardet, Jean-Charles Faugère, Bruno Salvy, and Bo-Yin Yang. Asymptotic behaviour of the degree of regularity of semi-regular polynomial systems. In The Effective Methods in Algebraic Geometry Conference - MEGA 2005, pages $1-14,2005$.

[BG13] Manuel Bluhm and Shay Gueron. Fast software implementation of binary elliptic curve cryptography. IACR Cryptology ePrint Archive, 2013:741, 2013.

[BGTZ08] Richard P. Brent, Pierrick Gaudry, Emmanuel Thomé, and Paul Zimmermann. Faster multiplication in $\mathrm{gf}(2)[\mathrm{x}]$. In Algorithmic Number Theory, 8th International Symposium, ANTS-VIII, Banff, Canada, May 17-22, 2008, Proceedings, pages 153-166, 2008.

$\left[\mathrm{CCC}^{+}\right.$09] Anna Inn-Tung Chen, Ming-Shing Chen, Tien-Ren Chen, Chen-Mou Cheng, Jintai Ding, Eric Li-Hsiang Kuo, Frost Yu-Shuang Lee, and Bo-Yin Yang. SSE implementation of multivariate pkcs on modern x86 cpus. In Cryptographic Hardware and Embedded Systems - CHES 2009, 11th International Workshop, Lausanne, Switzerland, September 6-9, 2009, Proceedings, pages 33-48, 2009.

[CCK $\left.{ }^{+} 17\right]$ Ming-Shing Chen, Chen-Mou Cheng, Po-Chun Kuo, Wen-Ding Li, and Bo-Yin Yang. Faster multiplication for long binary polynomials. CoRR, abs/1708.09746, 2017.

$\left[\mathrm{CDP}^{+}{ }^{17}\right]$ M. Chen, J. Ding, A. Petzoldt, D. Schmidt, and B. Yang. Gui. Submission to NIST Post-Quantum Cryptography Standardization Process, 2017. https://csrc.nist.gov/projects/post-quantum-cryptography/ round-1-submissions.

$\left[\mathrm{CFMR}^{+} 17\right]$ A. Casanova, J.-C. Faugère, G. Macario-Rat, J. Patarin, L. Perret, and J. Ryckeghem. GeMSS: A great multivariate short signature. Submission to NIST Post-Quantum Cryptography Standardization Process, 2017. https://csrc.nist.gov/projects/post-quantum-cryptography/ round-1-submissions. 
[CHR ${ }^{+}$16] Ming-Shing Chen, Andreas Hülsing, Joost Rijneveld, Simona Samardjiska, and Peter Schwabe. From 5-pass $M Q$-based identification to $M Q$-based signatures. In Advances in Cryptology - ASIACRYPT 2016 - 22nd International Conference on the Theory and Application of Cryptology and Information Security, Hanoi, Vietnam, December 4-8, 2016, Proceedings, Part II, pages 135-165, 2016.

$\left[\mathrm{CLP}^{+}{ }^{18}\right] \quad$ Ming-Shing Chen, Wen-Ding Li, Bo-Yuan Peng, Bo-Yin Yang, and Chen-Mou Cheng. Implementing 128-bit secure MPKC signatures. IEICE Transactions, 101-A(3):553-569, 2018.

[Cou03] Nicolas Courtois. Generic attacks and the security of quartz. In Public Key Cryptography, volume 2567 of Lecture Notes in Computer Science, pages 351-364. Springer, 2003.

[DG17] Nir Drucker and Shay Gueron. A toolbox for software optimization of QC-MDPC code-based cryptosystems. IACR Cryptology ePrint Archive, 2017:1251, 2017.

[DPP16] James H. Davenport, Christophe Petit, and Benjamin Pring. A generalised successive resultants algorithm. In Sylvain Duquesne and Svetla PetkovaNikova, editors, Arithmetic of Finite Fields - 6th International Workshop, WAIFI 2016, Ghent, Belgium, July 13-15, 2016, Revised Selected Papers, volume 10064 of Lecture Notes in Computer Science, pages 105-124, 2016.

[FJ03] Jean-Charles Faugère and Antoine Joux. Algebraic cryptanalysis of hidden field equation (HFE) cryptosystems using gröbner bases. In Dan Boneh, editor, Advances in Cryptology - CRYPTO 2003, 23rd Annual International Cryptology Conference, Santa Barbara, California, USA, August 17-21, 2003, Proceedings, volume 2729 of Lecture Notes in Computer Science, pages 44-60. Springer, 2003.

[FPR17] J.-C. Faugère, L. Perret, and J. Ryckeghem. DualModeMS: A dual mode for multivariate-based signature. Submission to NIST Post-Quantum Cryptography Standardization Process, 2017. https://csrc.nist.gov/projects/ post-quantum-cryptography/round-1-submissions.

[Ga02] Torbjörn Granlund and al. GNU Multiple Precision Arithmetic Library 6.1.2, December 2002. https://gmplib.org/.

[GBA13] M. Peeters G. Bertoni, J. Daemen and G. Van Assche. A software interface for keccak. 2013.

[GK03] Johann Großschädl and Guy-Armand Kamendje. Instruction set extension for fast elliptic curve cryptography over binary finite fields gf $(2 \mathrm{~m})$. In 14 th IEEE International Conference on Application-Specific Systems, Architectures, and Processors (ASAP 2003), 24-26 June 2003, The Hague, The Netherlands, page 455. IEEE Computer Society, 2003.

[GM10] Shuhong Gao and Todd D. Mateer. Additive fast fourier transforms over finite fields. IEEE Trans. Information Theory, 56(12):6265-6272, 2010.

[GvdHL15] Bruno Grenet, Joris van der Hoeven, and Grégoire Lecerf. Randomized root finding over finite fft-fields using tangent graeffe transforms. In Kazuhiro Yokoyama, Steve Linton, and Daniel Robertz, editors, Proceedings of the 2015 $A C M$ on International Symposium on Symbolic and Algebraic Computation, ISSAC 2015, Bath, United Kingdom, July 06 - 09, 2015, pages 197-204. ACM, 2015 . 
[Har10] William B. Hart. Fast library for number theory: An introduction. In Mathematical Software - ICMS 2010, Third International Congress on Mathematical Software, Kobe, Japan, September 13-17, 2010. Proceedings, pages 88-91, 2010.

[HMV03] Darrel Hankerson, Alfred J. Menezes, and Scott Vanstone. Guide to Elliptic Curve Cryptography. Springer-Verlag New York, Inc., Secaucus, NJ, USA, 2003.

[Int18] Intel architecture instruction set extensions programming reference, May 2018. https://software.intel.com/sites/default/files/managed/c5/15/ architecture-instruction-set-extensions-programming-reference . pdf.

[IT88] Toshiya Itoh and Shigeo Tsujii. A fast algorithm for computing multiplicative inverses in $\operatorname{gf}\left(2^{\mathrm{m}}\right)$ using normal bases. Inf. Comput., 78(3):171-177, 1988.

[KPG99] Aviad Kipnis, Jacques Patarin, and Louis Goubin. Unbalanced oil and vinegar signature schemes. In Jacques Stern, editor, Advances in Cryptology EUROCRYPT '99, International Conference on the Theory and Application of Cryptographic Techniques, Prague, Czech Republic, May 2-6, 1999, Proceeding, volume 1592 of Lecture Notes in Computer Science, pages 206-222. Springer, 1999.

[LN96] Rudolf Lidl and Harald Niederreiter. Finite Fields. Encyclopedia of Mathematics and its Applications. Cambridge University Press, 2 edition, 1996.

[Mai15] Jeremy Maitin-Shepard. Optimal software-implemented itoh-tsujii inversion for $\operatorname{gf}\left(2^{\mathrm{m}}\right)$. IACR Cryptology ePrint Archive, 2015:28, 2015.

[Mon05] Peter L. Montgomery. Five, six, and seven-term karatsuba-like formulae. IEEE Trans. Computers, 54(3):362-369, 2005.

[MQs18] MQsoft: a fast multivariate cryptography library, December 2018. https: //www-polsys.lip6.fr/Links/NIST/MQsoft.html.

[MS17] Alexander Maximov and Helena Sjoberg. On fast multiplication in binary finite fields and optimal primitive polynomials over GF(2). IACR Cryptology ePrint Archive, 2017:889, 2017.

[oST17] National Institute of Standards and Technology. Round 1 submissions - postquantum cryptography | csrc, 2017. https://csrc.nist.gov/projects/ post-quantum-cryptography/round-1-submissions.

[Pat96] Jacques Patarin. Hidden fields equations (HFE) and isomorphisms of polynomials (IP): two new families of asymmetric algorithms. In Ueli M. Maurer, editor, Advances in Cryptology - EUROCRYPT' '96, International Conference on the Theory and Application of Cryptographic Techniques, Saragossa, Spain, May 12-16, 1996, Proceeding, volume 1070 of Lecture Notes in Computer Science, pages 33-48. Springer, 1996.

$\left[\mathrm{PCY}^{+} 15\right] \quad$ Albrecht Petzoldt, Ming-Shing Chen, Bo-Yin Yang, Chengdong Tao, and Jintai Ding. Design principles for hfev- based multivariate signature schemes. In Tetsu Iwata and Jung Hee Cheon, editors, Advances in Cryptology - ASIACRYPT 2015 - 21st International Conference on the Theory and Application of Cryptology and Information Security, Auckland, New Zealand, November 29 - December 3, 2015, Proceedings, Part I, volume 9452 of Lecture Notes in Computer Science, pages 311-334. Springer, 2015. 
[Pet14] Christophe Petit. Finding roots in $\operatorname{gf}\left(\mathrm{p}^{\mathrm{n}}\right)$ with the successive resultant algorithm. IACR Cryptology ePrint Archive, 2014:506, 2014.

[Sho03] Victor Shoup. Ntl: A library for doing number theory. 012003. http: //www. shoup.net/ntl/.

[Swa62] Richard G. Swan. Factorization of polynomials over finite fields. Pacific J. Math., 12(3):1099-1106, 1962.

$\left[\mathrm{TFA}^{+} 11\right]$ Jonathan Taverne, Armando Faz-Hernández, Diego F. Aranha, Francisco Rodríguez-Henríquez, Darrel Hankerson, and Julio López. Software implementation of binary elliptic curves: impact of the carry-less multiplier on scalar multiplication. IACR Cryptology ePrint Archive, 2011:170, 2011.

[vzGG13] Joachim von zur Gathen and Jürgen Gerhard. Modern Computer Algebra (3. ed). Cambridge University Press, 2013. 


\section{Appendix}

\section{A Modular Reduction by a Pentanomial}

For completeness, we explain here the principle of modular reduction by a pentanomial. Let $f_{5}(x)=x^{n}+x^{k_{3}}+x^{k_{2}}+x^{k_{1}}+1$ such that $0<k_{1}<k_{2}<k_{3} \leq\left\lceil\frac{n}{2}\right\rceil$. Let $R_{0}=\sum_{i=0}^{n-1} r_{i} x^{i}$, $R_{k_{j}}=\sum_{i=n}^{2 n-k_{j}-1} r_{i} x^{i-n}$ and $S_{k_{j}}=\sum_{i=2 n-k_{j}}^{2 n-2} r_{i} x^{i-2 n+k_{j}}$ for $j \in \llbracket 1,3 \rrbracket$, we have:

$$
R=R_{0}+\left(R_{k_{j}}+S_{k_{j}} x^{n-k_{j}}\right) x^{n} .
$$

We do a first step of reduction by $f_{5}$ by replacing $x^{n}$ by $f_{5}(x)-x^{n}$ in Equation (9). To compute $\left(R_{k_{j}}+S_{k_{j}} x^{n-k_{j}}\right) \times\left(1+x^{k_{1}}+x^{k_{2}}+x^{k_{3}}\right)$, we multiply the left operand by $x^{k_{j}}$ for $j \in \llbracket 1,3 \rrbracket$, and we choose $j=1$ when we multiply the left operand by 1 . We obtain:

$R=R_{0}+\left(R_{k_{1}}+S_{k_{1}} x^{n-k_{1}}\right)+\left(R_{k_{1}} x^{k_{1}}+S_{k_{1}} x^{n}\right)+\left(R_{k_{2}} x^{k_{2}}+S_{k_{2}} x^{n}\right)+\left(R_{k_{3}} x^{k_{3}}+S_{k_{3}} x^{n}\right) \bmod f_{5}$.

We iterate a new step of reduction:

$R=R_{0}+R_{k_{1}}+S_{k_{1}} x^{n-k_{1}}+R_{k_{1}} x^{k_{1}}+R_{k_{2}} x^{k_{2}}+R_{k_{3}} x^{k_{3}}+\left(S_{k_{1}}+S_{k_{2}}+S_{k_{3}}\right)\left(f_{5}(x)-x^{n}\right) \bmod f_{5}$.

In Equation (10), the degree of $R$ is $\max \left(n-1,2\left(k_{3}-1\right)\right)$. So, $R$ is reduced modulo $f_{5}$ only if $2\left(k_{3}-1\right)<n$. In two steps of reduction, we have then a method to compute the modular reduction for all pentanomials such that $2\left(k_{3}-1\right)<n$.

To optimize the computation of (10), we compute $R_{k_{1}}+R_{k_{1}} x^{k_{1}}+R_{k_{2}} x^{k_{2}}+R_{k_{3}} x^{k_{3}}$ as $R_{k_{1}}\left(f_{5}(x)-x^{n}\right) \bmod x^{n}$. In this way, we can rewrite $R$ as:

$$
R=R_{0}+S_{k_{1}} x^{n-k_{1}}+\left(\left(R_{k_{1}}+S_{k_{1}}+S_{k_{2}}+S_{k_{3}}\right)\left(f_{5}(x)-x^{n}\right) \bmod x^{n}\right) \bmod f_{5}
$$

The product $\left(R_{k_{1}}+S_{k_{1}}+S_{k_{2}}+S_{k_{3}}\right)\left(f_{5}(x)-x^{n}\right)$ can be computed with same methods that in Section 2.3: directly with several calls to PCLMULQDQ instructions, or else with the shift-and-add strategy. For the moment, our library uses the shift-and-add strategy which has the advantage to be portable since it does not require PCLMULQDQ.

The parameters of Gui and DualModeMS256 require the use of irreducible pentanomials. For $n \in\{184,312,448,544\}$, we have choose respectively $x^{184}+x^{27}+x^{24}+x+1, x^{312}+$ $x^{128}+x^{15}+x^{5}+1, x^{448}+x^{64}+x^{39}+x^{33}+1$ and $x^{544}+x^{128}+x^{3}+x+1$. For these values of $n, n$ is a multiple of 8 . We speed-up the extraction of $R_{k_{1}}$ from $R$ by using shifts of bytes (PSRLDQ and PSHUFB instructions). For $n=184$, we choose $k_{2}=24$ because it is a multiple of 8 . This permits to improve the multiplication by $x^{24}$ by using shifts of bytes (PSLLDQ and PSHUFB instructions). For $n=312$ and $n=544$, we choose $k_{3}=128$. This improve the multiplication of $R_{k_{1}}$ by $x^{128}$, which does not require shifts when the data are stored on 64 -bit or 128 -bit registers. For $n=448$, we choose $k_{3}=64$ because $n$ is a multiple of 64 . We use this to simplify the multiplication of $R_{k_{1}}$ by $x^{64}$ : instead of doing this computation, we remark that the result is already available in the input. The result is aligned on 128 bits in $R$. For $n=184$ and $n=544$, the choice of $k_{1}=1$ implies $S_{k_{1}}=0$. This saves one instruction in our implementation. 


\section{B Addition Chains for the ITMIA}

Table 25: Proposed addition chains to minimize the number of multiplications in $\mathbb{F}_{2^{n}}$. The bold numbers are used to create some numbers of the chain.

\begin{tabular}{|l|l|l|l|}
\hline$n$ & addition chains & nb of mul. & used in Algorithm 5 \\
\hline 174 & $\mathbf{1}, 2,4, \mathbf{5}, 10,20,21,42,84,168,173$ & 10 & no \\
\hline 184 & $\mathbf{1}, 2, \mathbf{3}, \mathbf{4}, \mathbf{7}, 11,22,44,88,176,183$ & 10 & no \\
\hline 185 & $\mathbf{1}, 2,4,5,10,11,22,23,46,92,184$ & 10 & yes \\
\hline 265 & $\mathbf{1}, 2,4,8,16,32,33,66,132,264$ & 9 & yes \\
\hline 266 & $\mathbf{1}, 2,4,8,16,32,33,66,132,264,265$ & 10 & yes \\
\hline 312 & $\mathbf{1}, \mathbf{2}, 4, \mathbf{5}, \mathbf{7}, 14,19,38,76,152,304,311$ & 11 & no \\
\hline 313 & $\mathbf{1}, 2,3, \mathbf{6}, 12,18,36,72,78,156,312$ & 10 & no \\
\hline 354 & $\mathbf{1}, 2,4,5,10,11,22,44,88,176,352,353$ & 11 & yes \\
\hline 402 & $\mathbf{1}, 2,3,6,12,24,25,50,100,200,400,401$ & 11 & yes \\
\hline 448 & $\mathbf{1}, 2, \mathbf{3}, 6,12,24,27,54,108,111,222,444,447$ & 12 & no \\
\hline 544 & $\mathbf{1}, 2, \mathbf{3}, 6,12, \mathbf{1 5}, 30,33,66,132,264,528,543$ & 12 & no \\
\hline
\end{tabular}

\section{Polynomial Multiplication in $\mathbb{F}_{2^{n}}[X]$}

Fast algorithms which are presented in Appendix D and Section 3.6 require fast multiplications in $\mathbb{F}_{2^{n}}[X]$. The main fast multiplication is the Karatsuba method [vzGG13, Section 8.1]. The fast convolution algorithm [vzGG13, Algorithm 8.16] that uses Fast Fourier Transform (FFT) cannot be used here because primitive $2^{k}$-th roots of unity do not exist in the binary field. However, efficient additive FFT in characteristic two have been proposed [GM10, BCS13], but they are probably not invertible or not enough efficient to be used here.

Karatsuba multiplication is well known when the degree of polynomials is a power of two [vzGG13, Algorithm 8.1], and can be easily extended for all degrees. However, fast algorithms require multiplications of two polynomials having different degrees. To multiply $A, B \in \mathbb{F}_{2^{n}}[X]$ respectively of degree $d a, d b$ such that $d a \geq d b$, we just split $A$ by block of size $d b+1$ in order to apply the multiplication of each block by $B$. The classical and Karatsuba multiplications in $\mathbb{F}_{2^{n}}[X]$ are available in MQsoft.

\section{Fast Euclidean Division in $\mathbb{F}_{2^{n}}[X]$}

In this section, we define $\operatorname{Rec}_{i}(P)$ the reciprocal polynomial of $P \in \mathbb{F}_{2^{n}}[X]$, such that $\operatorname{Rec}_{i}(P)=X^{i} P\left(\frac{1}{X}\right)$. The fast Euclidean division [vzGG13, Algorithm 9.5] of $A$ by $F$ consists to write $A=F Q+R$ with $Q, R \in \mathbb{F}_{2^{n}}[X]$, and to remark that:

$$
\operatorname{Rec}_{2 D-2}(A)=\operatorname{Rec}_{D}(F) \operatorname{Rec}_{D-2}(Q)+X^{D-1} \operatorname{Rec}_{D-1}(R) .
$$

Because the degree of $Q$ is at most $D-2$, we can compute Equation (12) modulo $X^{D-1}$. So, we obtain the following formula for $Q$ :

$$
\operatorname{Rec}_{D-2}(Q)=\operatorname{Rec}_{2 D-2}(A) \operatorname{Rec}_{D}(F)^{-1} \bmod X^{D-1} .
$$

Once $Q$ is known, $R$ is easily obtained. This process can be summarized in three steps:

- Computation of $\operatorname{Rec}_{D}(F)^{-1} \bmod X^{D-1}$ with Newton iterations [vzGG13, Algorithm $9.3]$. 
- Computation of the quotient with Equation (13).

- Computation of the remainder: $R=A-Q F \bmod X^{D}$.

The fast Euclidean division can be used to compute the Frobenius map (Section 3.5). In the case of HFE-, $\operatorname{Rec}_{D}(F)^{-1} \bmod X^{D-1}$ can be precomputed and stored with the secret-key, because the computation does not have dependences with the constant of $F$ (in Section 1.3 , we are looking for a root of $\left.F(X, \mathbf{v})-D^{\prime}\right)$. For the vinegar variant, the linear terms of $F$ depend on the choice of vinegars variables and so $\operatorname{Rec}_{D}(F)^{-1} \bmod X^{D-1}$ must be computed for each of these choices. So, a precomputed table requires $2^{v}(D-1) n$ bits, which huge.

However, when $D$ is odd, $D-1$ is a power of two and so the computation of $\operatorname{Rec}_{D}(F)^{-1} \bmod$ $X^{D-1}$ can be improved. Because $F$ has a HFE structure, we have that:

$$
\operatorname{Rec}_{D}(F)=1+\operatorname{coef}_{D}(F) \bmod X^{\frac{D-1}{4}},
$$

and so:

$$
\operatorname{Rec}_{D}(F)^{-1}=\sum_{i=0}^{\frac{D-1}{4}-1} \operatorname{coef}_{D}(F)^{i} X^{i} \bmod X^{\frac{D-1}{4}} .
$$

With this formula, only two iterations of Newton are required to compute $\operatorname{Rec}_{D}(F)^{-1} \bmod$ $X^{D-1}$ (by computing $\operatorname{Rec}_{D}(F)^{-1}$ modulo $X^{\frac{D-1}{2}}$ then modulo $X^{D-1}$ ). The two others steps require only two multiplications in $\mathbb{F}_{2^{n}}[X]$ modulo a power of $X$. With a enough fast multiplication, fast algorithm is better asymptotically than classical Euclidean division (Section 3.3). However, a fast Euclidean division based on Karatsuba multiplication (Appendix C) is inefficient compared to the classical Euclidean division which exploits the sparse structure of $F$. This explains why NTL is slow to compute the Frobenius map in Section 3.5.

In the general case, the fast Euclidean division algorithm is interesting. It permits to improve the fast GCD algorithm in Section 3.6. The fast Euclidean division is implemented in MQsoft, as well as the fast Frobenius map, the fast GCD, and so the fast root finding algorithm. This is slower for HFE polynomials, but is efficient in the general case.

\section{E Performance of the NIST submissions}

Table 26: Number of mega cycles for each cryptographic operation (best implementation provided for the NIST submissions). We use a Haswell processor (ServerH). Turbo Boost is not used.

\begin{tabular}{|c|c|c|c|c|c|}
\hline scheme & sec. level & $(n, D, \Delta, v, \mathrm{nb}$ ite $)$ & key gen. & signature gen. & signature verif. \\
\hline GeMSS128 & 128 & $(174,513,12,12,4)$ & 125 & 1510 & 0.161 \\
\hline GeMSS192 & 192 & $(265,513,22,20,4)$ & 562 & 3870 & 0.439 \\
\hline GeMSS256 & 256 & $(354,513,30,33,4)$ & 1620 & 7300 & 1.01 \\
\hline Gui-184 & 112 & $(184,33,16,16,2)$ & 744 & 17.1 & 0.0869 \\
\cline { 2 - 6 } & 126 & $(184,33,16,16,3)$ & 744 & 26.1 & 0.131 \\
\cline { 2 - 6 } & 128 & $(184,33,16,16,4)$ & 744 & 34.1 & 0.177 \\
\hline Gui-312 & 192 & $(312,129,24,20,2)$ & 5180 & 505 & 0.278 \\
\hline Gui-448 & 256 & $(448,513,32,28,2)$ & 30500 & 17600 & 0.715 \\
\hline Inner.DualModeMS128 & 128 & $(266,129,10,11,1)$ & 451 & 146 & 0.0925 \\
\hline
\end{tabular}


Table 27: Number of mega cycles for each cryptographic operation (best implementation provided for the NIST submissions). We use a Haswell processor (DesktopH).

\begin{tabular}{|c|c|c|c|c|}
\hline scheme & $(n, D, \Delta, v, \mathrm{nb}$ _ite $)$ & key gen. & signature gen. & signature verif. \\
\hline GeMSS128 & $(174,513,12,12,4)$ & 112 & 1360 & 0.145 \\
\hline GeMSS192 & $(265,513,22,20,4)$ & 505 & 3480 & 0.394 \\
\hline GeMSS256 & $(354,513,30,33,4)$ & 1460 & 6580 & 0.911 \\
\hline Gui-184 & $(184,33,16,16,4)$ & 670 & 30.5 & 0.16 \\
\hline Gui-312 & $(312,129,24,20,2)$ & 4660 & 452 & 0.25 \\
\hline Gui-448 & $(448,513,32,28,2)$ & 27500 & 15800 & 0.653 \\
\hline Inner.DualModeMS128 & $(266,129,10,11,1)$ & 406 & 131 & 0.0831 \\
\hline DualModeMS128 & $(266,129,10,11,1)$ & 1670000 & 8780 & 8.23 \\
\hline
\end{tabular}

Table 28: Number of mega cycles for each cryptographic operation (best implementation provided for the NIST submissions). We use a Skylake processor (DesktopS).

\begin{tabular}{|c|c|c|c|c|}
\hline scheme & $(n, D, \Delta, v, \mathrm{nb}$ _ite $)$ & key gen. & signature gen. & signature verif. \\
\hline GeMSS128 & $(174,513,12,12,4)$ & 92.6 & 978 & 0.125 \\
\hline GeMSS192 & $(265,513,22,20,4)$ & 424 & 2570 & 0.35 \\
\hline GeMSS256 & $(354,513,30,33,4)$ & 1150 & 4040 & 0.883 \\
\hline Gui-184 & $(184,33,16,16,4)$ & 612 & 22.1 & 0.141 \\
\hline Gui-312 & $(312,129,24,20,2)$ & 4110 & 323 & 0.233 \\
\hline Gui-448 & $(448,513,32,28,2)$ & 26600 & 10900 & 0.612 \\
\hline Inner.DualModeMS128 & $(266,129,10,11,1)$ & 349 & 97.7 & 0.0756 \\
\hline DualModeMS128 & $(266,129,10,11,1)$ & 1580000 & 6530 & 7.79 \\
\hline
\end{tabular}

\section{F Performance of MQsoft}

Table 29: Number of mega cycles (Mc) for each cryptographic operation with our library for a Haswell processor (ServerH), followed by the speed-up between the best implementation provided for the NIST submissions versus our implementation. For example, $45 \delta:+180 \%$ means a performance of $45 \mathrm{Mc}$ with MQsoft, and a performance of $45 \times 2.8=130$ Mc for the NIST implementations.

\begin{tabular}{|c|c|cc|cc|c|c|}
\hline scheme & $(n, D, \Delta, v, \mathrm{nb}$ ite $)$ & \multicolumn{2}{|c|}{ key gen. } & \multicolumn{2}{|c|}{ signature gen. } & \multicolumn{2}{c|}{ signature verif. } \\
\hline GeMSS128 & $(174,513,12,12,4)$ & 45 & $\delta:+180 \%$ & 960 & $\delta:+57 \%$ & 0.081 & $\delta:+98 \%$ \\
\hline GeMSS192 & $(265,513,22,20,4)$ & 230 & $\delta:+140 \%$ & 3100 & $\delta:+25 \%$ & 0.24 & $\delta:+83 \%$ \\
\hline GeMSS256 & $(354,513,30,33,4)$ & 690 & $\delta:+130 \%$ & 5900 & $\delta:+23 \%$ & 0.58 & $\delta:+75 \%$ \\
\hline Gui-184 & $(184,33,16,16,4)$ & 57 & $\delta:+1200 \%$ & 24 & $\delta:+40 \%$ & 0.11 & $\delta:+67 \%$ \\
\hline Gui-185 & $(185,33,16,16,4)$ & 58 & $\delta:+1200 \%$ & 20 & $\delta:+73 \%$ & 0.1 & $\delta:+74 \%$ \\
\hline Gui-312 & $(312,129,24,20,2)$ & 370 & $\delta:+1300 \%$ & 440 & $\delta:+14 \%$ & 0.17 & $\delta:+61 \%$ \\
\hline Gui-313 & $(313,129,24,20,2)$ & 380 & $\delta:+1300 \%$ & 420 & $\delta:+21 \%$ & 0.17 & $\delta:+59 \%$ \\
\hline Gui-448 & $(448,513,32,28,2)$ & 1600 & $\delta:+1800 \%$ & 7100 & $\delta:+150 \%$ & 0.45 & $\delta:+59 \%$ \\
\hline Inner.DualModeMS128 & $(266,129,10,11,1)$ & 210 & $\delta:+120 \%$ & 110 & $\delta:+30 \%$ & 0.045 & $\delta:+110 \%$ \\
\hline DualModeMS128 & $(266,129,10,11,1)$ & 1900000 & $\delta:+0 \%$ & 7400 & $\delta:+32 \%$ & 9.2 & $\delta:+0 \%$ \\
\hline
\end{tabular}


Table 30: Number of mega cycles for each cryptographic operation with MQsoft. We use a Haswell processor (ServerH). Turbo Boost is not used.

\begin{tabular}{|c|c|c|c|c|}
\hline scheme & $(n, D, \Delta, v, \mathrm{nb}$ _ite $)$ & key gen. & signature gen. & signature verif. \\
\hline GeMSS128 & $(174,513,12,12,4)$ & 44.9 & 962 & 0.0814 \\
\hline GeMSS192 & $(265,513,22,20,4)$ & 235 & 3080 & 0.24 \\
\hline GeMSS256 & $(354,513,30,33,4)$ & 694 & 5930 & 0.577 \\
\hline Gui-184 & $(184,33,16,16,4)$ & 57.2 & 24.4 & 0.106 \\
\hline Gui-185 & $(185,33,16,16,4)$ & 58.4 & 19.8 & 0.102 \\
\hline Gui-312 & $(312,129,24,20,2)$ & 373 & 444 & 0.172 \\
\hline Gui-313 & $(313,129,24,20,2)$ & 380 & 416 & 0.174 \\
\hline Gui-448 & $(448,513,32,28,2)$ & 1570 & 7070 & 0.451 \\
\hline Inner.DualModeMS128 & $(266,129,10,11,1)$ & 210 & 112 & 0.0446 \\
\hline Inner.DualModeMS192 & $(402,129,18,18,1)$ & 1030 & 245 & 0.143 \\
\hline Inner.DualModeMS256 & $(544,129,32,32,1)$ & 4000 & 487 & 0.263 \\
\hline DualModeMS128 & $(266,129,10,11,1)$ & $1.85 \mathrm{M}$ & 7360 & 9.16 \\
\hline DualModeMS192 & $(402,129,18,18,1)$ & $7.14 \mathrm{M}$ & 24700 & 17.1 \\
\hline DualModeMS256 & $(544,129,32,32,1)$ & $18 \mathrm{M}$ & 131000 & 28.5 \\
\hline
\end{tabular}

Table 31: Number of mega cycles for each cryptographic operation with MQsoft. We use a Haswell processor (DesktopH).

\begin{tabular}{|c|c|c|c|c|}
\hline scheme & $(n, D, \Delta, v, \mathrm{nb}$ _ite $)$ & key gen. & signature gen. & signature verif. \\
\hline GeMSS128 & $(174,513,12,12,4)$ & 40.5 & 864 & 0.0722 \\
\hline GeMSS192 & $(265,513,22,20,4)$ & 211 & 2770 & 0.215 \\
\hline GeMSS256 & $(354,513,30,33,4)$ & 625 & 5330 & 0.518 \\
\hline Gui-184 & $(184,33,16,16,4)$ & 51.4 & 21.9 & 0.088 \\
\hline Gui-185 & $(185,33,16,16,4)$ & 52.4 & 17.8 & 0.0899 \\
\hline Gui-312 & $(312,129,24,20,2)$ & 336 & 399 & 0.152 \\
\hline Gui-313 & $(313,129,24,20,2)$ & 344 & 374 & 0.155 \\
\hline Gui-448 & $(448,513,32,28,2)$ & 1430 & 6350 & 0.409 \\
\hline Inner.DualModeMS128 & $(266,129,10,11,1)$ & 189 & 101 & 0.0396 \\
\hline Inner.DualModeMS192 & $(402,129,18,18,1)$ & 928 & 221 & 0.129 \\
\hline Inner.DualModeMS256 & $(544,129,32,32,1)$ & 3640 & 438 & 0.241 \\
\hline DualModeMS128 & $(266,129,10,11,1)$ & $1.67 \mathrm{M}$ & 6610 & 8.23 \\
\hline DualModeMS192 & $(402,129,18,18,1)$ & $6.42 \mathrm{M}$ & 22200 & 15.3 \\
\hline DualModeMS256 & $(544,129,32,32,1)$ & $16.2 \mathrm{M}$ & 119000 & 25.7 \\
\hline
\end{tabular}


Table 32: Number of mega cycles for each cryptographic operation with MQsoft. We use a Skylake processor (DesktopS).

\begin{tabular}{|c|c|c|c|c|}
\hline scheme & $(n, D, \Delta, v, \mathrm{nb}$ _ite $)$ & key gen. & signature gen. & signature verif. \\
\hline GeMSS128 & $(174,513,12,12,4)$ & 36.2 & 597 & 0.0666 \\
\hline GeMSS192 & $(265,513,22,20,4)$ & 189 & 1880 & 0.197 \\
\hline GeMSS256 & $(354,513,30,33,4)$ & 564 & 3100 & 0.451 \\
\hline Gui-184 & $(184,33,16,16,4)$ & 48.6 & 16.7 & 0.0811 \\
\hline Gui-185 & $(185,33,16,16,4)$ & 48.9 & 13.1 & 0.0811 \\
\hline Gui-312 & $(312,129,24,20,2)$ & 307 & 285 & 0.126 \\
\hline Gui-313 & $(313,129,24,20,2)$ & 310 & 260 & 0.128 \\
\hline Gui-448 & $(448,513,32,28,2)$ & 1340 & 4390 & 0.327 \\
\hline Inner.DualModeMS128 & $(266,129,10,11,1)$ & 171 & 68.5 & 0.0297 \\
\hline Inner.DualModeMS192 & $(402,129,18,18,1)$ & 850 & 153 & 0.0982 \\
\hline DualModeMS128 & $(266,129,10,11,1)$ & $1.58 \mathrm{M}$ & 4570 & 0.204 \\
\hline DualModeMS192 & $(402,129,18,18,1)$ & $5.72 \mathrm{M}$ & 15800 & 7.79 \\
\hline DualModeMS256 & $(544,129,32,32,1)$ & $14.4 \mathrm{M}$ & 80600 & 24.9 \\
\hline
\end{tabular}

